Florida International University FIU Digital Commons

2-9-2012

\title{
Temporal Mapping and Connectivity using NIRS for Language Related Tasks
}

Michael A. Hall

Florida International University, mhallo03@fiu.edu

DOI: $10.25148 /$ etd.FI12041111

Follow this and additional works at: https://digitalcommons.fiu.edu/etd

\section{Recommended Citation}

Hall, Michael A., "Temporal Mapping and Connectivity using NIRS for Language Related Tasks" (2012). FIU Electronic Theses and Dissertations. 560.

https://digitalcommons.fiu.edu/etd/560

This work is brought to you for free and open access by the University Graduate School at FIU Digital Commons. It has been accepted for inclusion in FIU Electronic Theses and Dissertations by an authorized administrator of FIU Digital Commons. For more information, please contact dcc@fiu.edu. 


\section{FLORIDA INTERNATIONAL UNIVERSITY}

Miami, Florida

TEMPORAL MAPPING AND CONNECTIVITY USING NIRS FOR LANGUAGE RELATED

TASKS

A thesis submitted in partial fulfillment of the

Requirements for the degree of

MASTER OF SCIENCE

in

BIOMEDICAL ENGINEERING

by

Michael A. Hall

2012 
To: Dean Amir Mirmiran

College of Engineering and Computing

This thesis, written by Michael A. Hall, and entitled Temporal Mapping and Connectivity using NIRS for Language Related Tasks, having been approved in respect to style and intellectual content, is referred to you for judgment.

We have read this thesis and recommend that it be approved.

Armando Barreto

Wei-Chiang Lin

Anuradha Godavarty, Major Professor

Date of Defense: February 9, 2012

The thesis of Michael A. Hall is approved.

Dean Amir Mirmiran

College of Engineering and Computing

Dean Lakshmi N. Reddi

University Graduate School

Florida International University, 2012 


\begin{abstract}
OF THE THESIS
TEMPORAL MAPPING AND CONNECTIVITY USING NIRS FOR LANGUAGE RELATED

TASKS

by
\end{abstract}

Michael A. Hall

Florida International University, 2012

Miami, Florida

Professor Anuradha Godavarty, Major Professor

Near infrared spectroscopy (NIRS) is an emerging neuroimaging modality with high temporal and good spatial resolution. In this thesis, NIRS was applied to understand functionality of the fronto-temporal cortex in response to language-related tasks. A 32-channel NIRS system (Imagent ISS Inc.) was used to perform experimental studies on 15 right-handed normal adults. Block-design based Word Expression and Word Reception paradigms were independently presented to participants. Activation, functional connectivity and cortical lateralization analyses were performed. From word expression studies, results showed left anterior region (encompassing Broca) is majorly involved over right homologue and posterior regions. From the word reception studies, results showed that right posterior region (encompassing right homologue of Wernicke) is highly involved in language reception, with right anterior region (encompassing right homologue of Broca) also involved. The current study has potential future applications in surgical evaluation of language regions in populations with neurological disorders such as epilepsy, and schizophrenia. 


\section{TABLE OF CONTENTS}

CHAPTER

PAGE

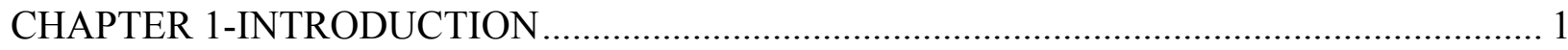

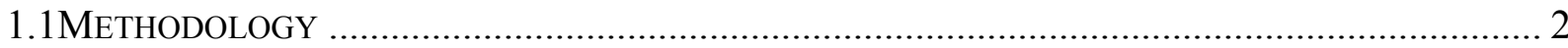

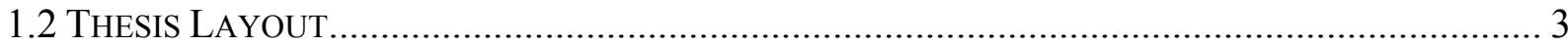

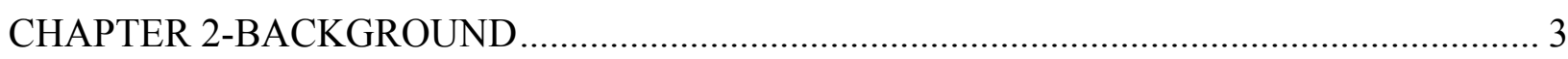

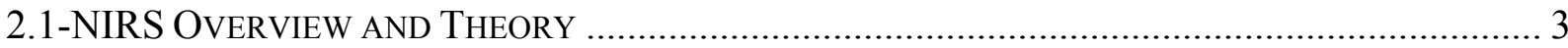

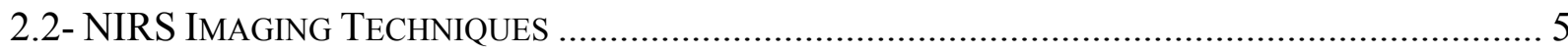

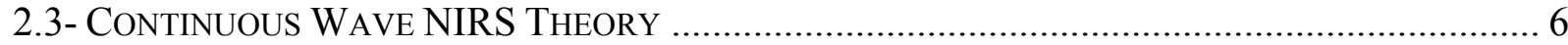

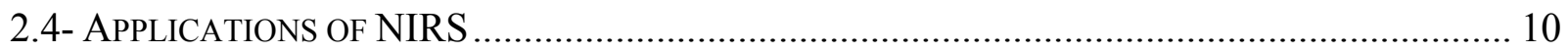

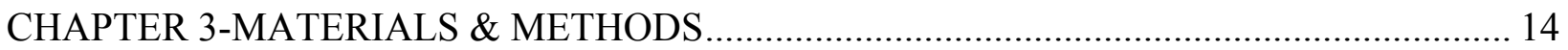

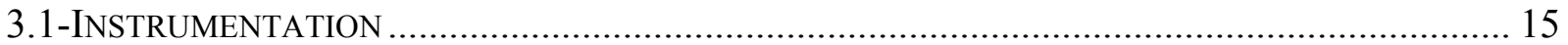

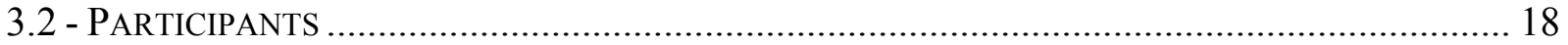

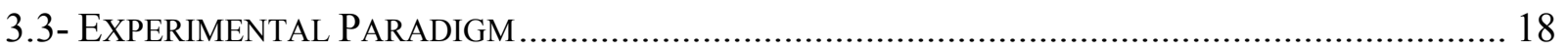

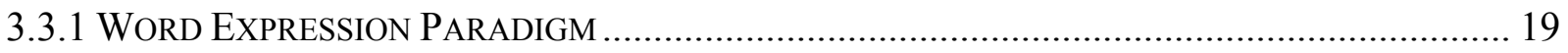

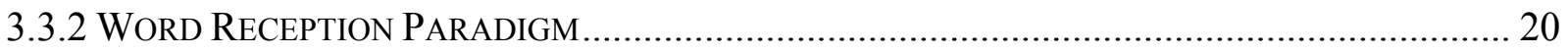

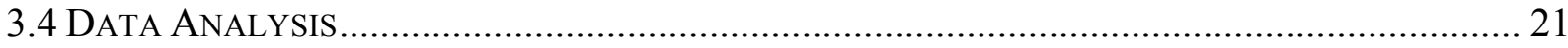

3.4.1 ACTIVATION ANALYSIS ...................................................................................... 26

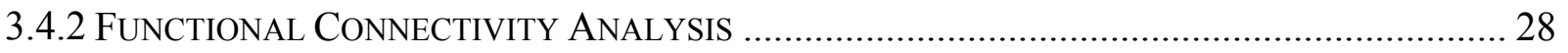

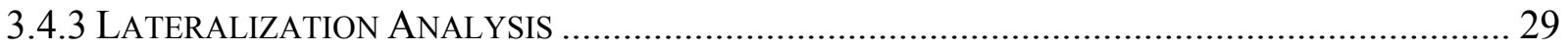

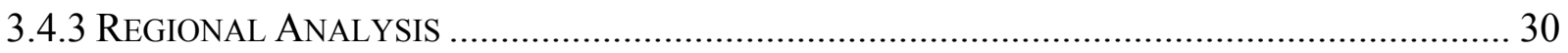

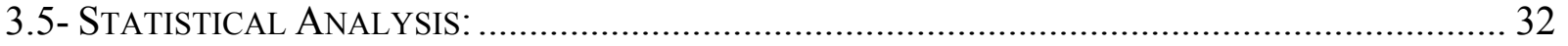

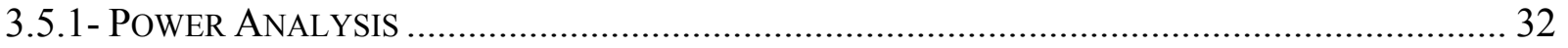

3.5.2- CONSISTENCY TEST: GRAND AVERAGE MEAN VS. INDIVIDUAL SUBJECT MEANS .............. 34

3.5.3- DifFERENCES IN HEMOdYNAMIC ReSPONSE ACRoss STIMULI ....................................... 34

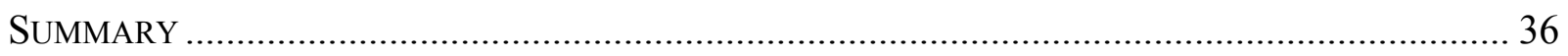

CHAPTER 4- RESULTS AND DISCUSSION: WORD EXPRESSION PARADIGM ……..... 37

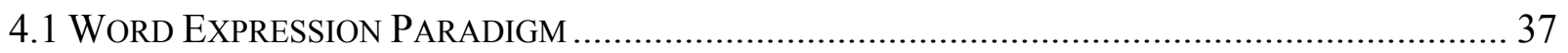

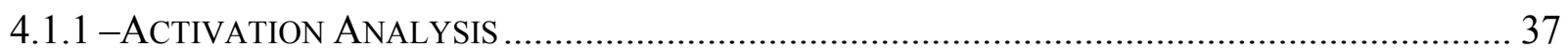

4.1.2 -FUNCTIONAL CONNECTIVITY ANALYSIS .................................................................. 43

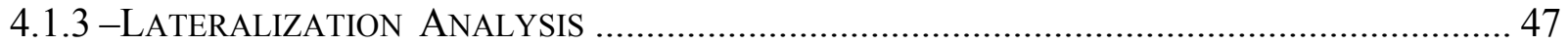

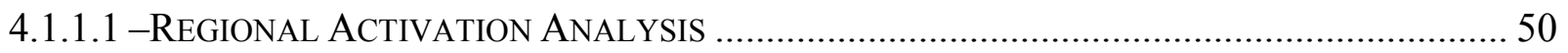

4.1.2.1 -REGIONAL FUNCTIONAL CONNECTIVITY ANALYSIS .................................................... 53

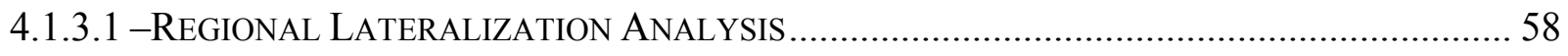

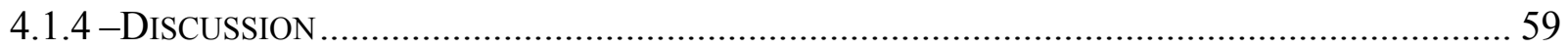

CHAPTER 5- RESULTS AND DISCUSSION: WORD RECEPTION PARADIGM ....................61

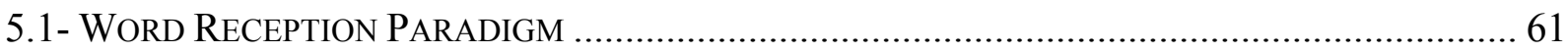

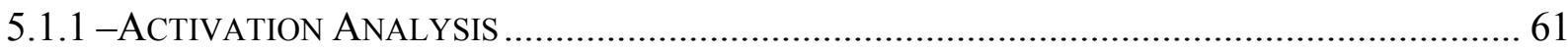

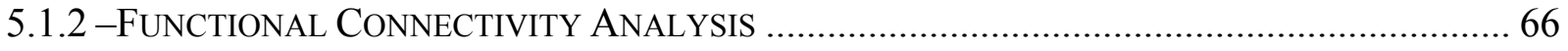


5.1.3 -LATERALIZATION ANALYSIS .

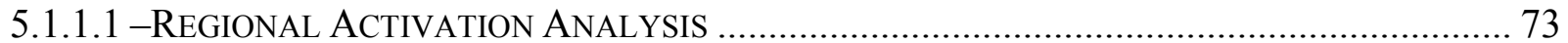

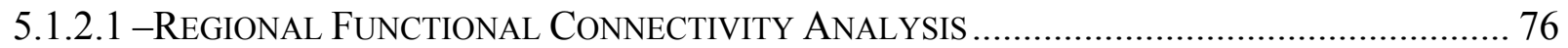

5.1.3.1 -REGIONAL LATERALIZATION ANALYSIS ............................................................ 81

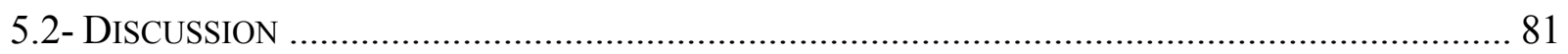

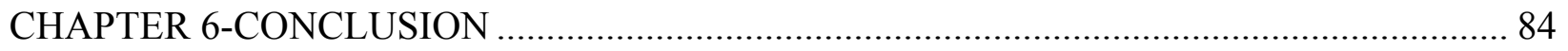

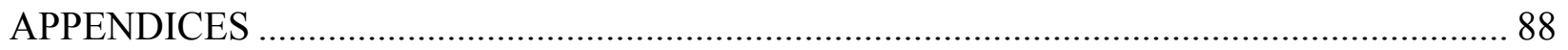

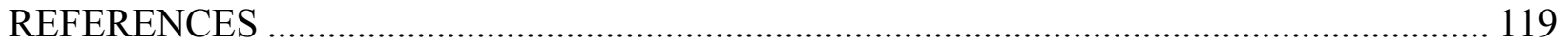




\section{LIST OF FIGURES}

FIGURE

PAGE

Figure 1 (a) Sensitivity of NIRS instrumentation, showing light propagating from a laser source to a detector several centimeters away, (b) NIRS probe used to measure primary motor cortex, (c) "Therapeutic window," Absorption spectrum of oxyhemoglobin and deoxyhemoglobin.( Huppert et. al $2009^{[2]}$.

Figure 2. Frequency domain based optical imager, Imagent (ISS, Inc., Champaign, IL). The Imagent was provided by Brain Institute at MCH (Miami Children's Hospital).

Figure 3. Source detector layout and Source detector placement based on International EEG 1020 placement.

Figure 4. A diagram of the (i) Word Expression Paradigm, and (ii)Word Reception Paradigm.

Figure 5. This figure shows the noise that is induced in the NIRS signal from respiratory cycles, and cardiac cycles.which are all part of the Mayer wave oscillation. Also to note is the motion artifact that is present during this study due to subject motion. (Huppert et. al (2009) ${ }^{[2]}$

Figure 6 Screen shot of the HomER program. The layout of the HomER program is based around an interactive graphical display of the NIRS probe, shown in the upper right (b). The user specifies this probe geometry within the data file imported into HomER as described in the text. By selecting source (displayed as " $x$ ") or detector ("o") positions on this probe layout, the user navigates through the display of their data. The original data are shown in (a) and the average evoked response is shown in (c).

Figure 7. Word Expression Paradigm activation plots. The plots show oxygenated (red) and deoxygenated (blue) hemoglobin activation in the left and right fronto-temporal regions of the brain during the Word Expression Paradigm. All plots are shown on the same scale. The y-axis corresponds to the relative change in hemoglobin, while time is shown on the x-axis. The plots are divided into the three 30 second stimuli (from left to right), Word Generation (W), Jaw Movement (J), Rest (R).

Figure 8. Oxy-hemoglobin Functional Connectivity Diagram. The diagram shows the correlation for channels 1-8 between the left and right hemisphere during the Word Expression Paradigm for $\mathrm{HbO}$. The boxes are shaded according to the scale on the right hand of the figure, which corresponds to the correlation values calculated for the left and right hemisphere during the Word Expression Paradigm. 
Figure 9. Deoxy-hemoglobin Functional Connectivity Diagram. The diagram shows the correlation for channels 1-8 between the left and right hemisphere during the Word Expression Paradigm for $\mathrm{HbR}$. The boxes are shaded according to the scale on the right hand of the figure, which corresponds to the correlation values calculated for the left and right hemisphere during the Word Expression Paradigm.

Figure 10. Total Hemoglobin Functional Connectivity Diagram. The diagram shows the correlation for channels 1-8 between the left and right hemisphere during the Word Expression Paradigm for HbT. The boxes are shaded according to the scale on the right hand of the figure, which corresponds to the correlation values calculated for the left and right hemisphere during the Word Expression Paradigm.

Figure 11. Lateralization Index Diagram. The following diagram shows the lateralization results for the three stimuli during the Word Expression Paradigm in the fronto-temporal region. The boxes that are shaded correspond to a dominate channel. Those boxes left unshaded correspond to a bilateral lateralization index (no dominance).

Figure 12. Regional Word Expression Paradigm activation plots. The plots show oxygenated (red) and deoxygenated (blue) hemoglobin activation in the left and right fronto-temporal regions of the brain during the Word Expression Paradigm. All plots are shown on the same scale. The yaxis corresponds to the relative change in hemoglobin, while time is shown on the x-axis. The plots are divided into the three 30 second stimuli (from left to right), Word Generation (W.G), Jaw Movement (J.M), Rest (R). The y-axis represents the relative concentration change in hemoglobin species (abu). The $\mathrm{x}$-axis represents time in seconds.

Figure 13. Oxy-hemoglobin Functional Connectivity Diagram. The diagram shows the correlation for the four regions between the left and right hemisphere during the regional Word Expression Paradigm for $\mathrm{HbO}$. The lines are shaded according to the scale on the right hand of the figure, which corresponds to the correlation values calculated for the left and right hemisphere during the Word Expression Paradigm.

Figure 14. Deoxy-hemoglobin Functional Connectivity Diagram. The diagram shows the correlation for the four regions between the left and right hemisphere during the Word Expression Paradigm for $\mathrm{HbR}$. The lines are shaded according to the scale on the right hand of the figure, which corresponds to the correlation values calculated for the left and right hemisphere during the Word Expression Paradigm.

Figure 15.Total Hemoglobin Functional Connectivity Diagram. The diagram shows the correlation for the four regions between the left and right hemisphere during the Word Expression Paradigm for HbT. The lines are shaded according to the scale on the right hand of the figure, which corresponds to the correlation values calculated for the left and right hemisphere during the Word Expression Paradigm. 
Figure 16. Lateralization Index Diagram. The following diagram shows the lateralization results for the three stimuli during the regional Word Expression Paradigm in the fronto-temporal region. The boxes that are shaded correspond to a dominate region. Those boxes left unshaded correspond to a bilateral lateralization index (no dominance).

Figure 17. Word Reception Paradigm activation plots. The plots show the oxygenated (red) and deoxygenated (blue) hemoglobin activation in the left and right fronto-temporal regions of the brain during the Word Expression Paradigm. All plots are shown on the same scale. The y-axis corresponds to the relative change in hemoglobin, while time is shown on the X-axis. The plots are divided into the three 30 second stimuli (from left to right), Forward Audio, Reverse Audio, Rest.

Figure 18. Oxy-hemoglobin Functional Connectivity Diagram. The diagram shows the correlation for channels 1-8 between the left and right hemisphere during the Word Reception Paradigm for $\mathrm{HbO}$. The boxes are shaded according to the scale on the right hand of the figure, which corresponds to the correlation values calculated for the left and right hemisphere during the Word Reception Paradigm.

Figure 19. Deoxy-hemoglobin Functional Connectivity Diagram. The diagram shows the correlation for channels 1-8 between the left and right hemisphere during the Word Reception Paradigm for $\mathrm{HbR}$. The boxes are shaded according to the scale on the right hand of the figure, which corresponds to the correlation values calculated for the left and right hemisphere during the Word Reception Paradigm.

Figure 20. Total hemoglobin Functional Connectivity Diagram. The diagram shows the correlation for channels 1-8 between the left and right hemisphere during the Word Reception Paradigm for HbT. The boxes are shaded according to the scale on the right hand of the figure, which corresponds to the correlation values calculated for the left and right hemisphere during the Word Reception Paradigm.

Figure 21. Lateralization Index Diagram. The following diagram shows the lateralization results for the three stimuli during the Word Reception Paradigm in the fronto-temporal region. The boxes that are shaded correspond to a dominate channel. Those boxes left unshaded correspond to a bilateral lateralization index (no dominance).

Figure 22. Regional Word Reception Paradigm activation plots. The plots show the oxygenated (red) and deoxygenated (blue) hemoglobin activation in the left and right fronto-temporal regions of the brain during the Word Expression Paradigm. All plots are shown on the same scale. The yaxis corresponds to the relative change in hemoglobin, while time is shown on the $\mathrm{x}$-axis. The plots are divided into the three 30 second stimuli (from left to right), Forward Audio (F.A), Reverse Audio (R.A), Rest (R). 
Figure 23. Oxy-hemoglobin Functional Connectivity Diagram. The diagram shows the correlation for the four regions between the left and right hemisphere during the regional Word Reception Paradigm for HbO. The lines are shaded according to the scale on the right hand of the figure, which corresponds to the correlation values calculated for the left and right hemisphere during the regional Word Reception Paradigm.

Figure 24. Deoxy-hemoglobin Functional Connectivity Diagram. The diagram shows the correlation for the four regions between the left and right hemisphere during the regional Word Reception Paradigm for HbR. The lines are shaded according to the scale on the right hand of the figure, which corresponds to the correlation values calculated for the left and right hemisphere during the regional Word Reception Paradigm.

Figure 25. Total hemoglobin Functional Connectivity Diagram. The diagram shows the correlation for the four regions between the left and right hemisphere during the regional Word Reception Paradigm for HbT. The lines are shaded according to the scale on the right hand of the figure, which corresponds to the correlation values calculated for the left and right hemisphere during the regional Word Reception Paradigm.

Figure 26. Lateralization Index Diagram. The following diagram shows the lateralization results for the three stimuli during the regional Word Reception Paradigm in the fronto-temporal region. The boxes that are shaded correspond to a dominate region. Those boxes left unshaded correspond to a bilateral lateralization index (no dominance).

Figure B1. Figure showing HomER GUI interface before any data has been imported to the GUI.

Figure B2. Figure showing HomER GUI interface with raw optical signal before filtering for right anterior fronto-temporal region.

Figure B3. Figure showing HomER GUI interface with dOD signal before filtering for right anterior fronto- temporal region.

Figure B4. Figure showing HomER GUI interface with dConc of $\mathrm{HbO}$ signal before filtering for right anterior fronto-temporal region. 96

Figure B5. Figure showing HomER GUI interface with raw intensity signal after $3^{\text {rd }}$ order bandpass filtration for right anterior fronto-temporal region.

Figure B6. Figure showing HomER GUI interface with dOD signal after $3^{\text {rd }}$ order bandpass filtration for right anterior fronto-temporal region.

Figure B7. Figure showing HomER GUI interface with dOD signal after $3^{\text {rd }}$ order bandpass filtration for right anterior fronto-temporal region. 
Figure B8. Figure showing HomER GUI interface with dConc of $\mathrm{HbO}$ signal after $3^{\text {rd }}$ order bandpass filtration for right anterior fronto-temporal region.

Figure B9. Figure showing HomER GUI interface with dOD of HbO signal after $3^{\text {rd }}$ order bandpass filtration, and averaging across 5 blocks to create a single block consisting of three thirty second stimuli for right anterior fronto- temporal region.

Figure B10. Figure showing HomER GUI interface with dOD of $\mathrm{HbO}$ signal after $3^{\text {rd }}$ order bandpass filtration, and averaging across 5 blocks to create a single block consisting of three thirty second stimuli for right anterior fronto-temporal region. 


\section{LIST OF TABLES}

Table 1: Work done to date on language function in the brain using NIRS. 12

Table 2: Different types of Biological signals, and their frequency range. 23

Table 3: Power Analysis look-up table based on Cohen's predictive values for a 95\% confidence interval. 33 Table 4: A table of the mean values for each of the three stimuli across all 16 channels for HbO, $\mathrm{HbR}$, and HbT during the Word Expression Paradigm. The red font indicates the channel which recorded the largest amplitude of change for both the left and right hemisphere.

Table 5: A table of the mean values for each of the three stimuli across all 4 regions for $\mathrm{HbO}$, $\mathrm{HbR}$, and $\mathrm{HbT}$ during the Word Expression Paradigm.

Table 6: A table of the mean values for each of the three stimuli across all 16 channels for $\mathrm{HbO}$, $\mathrm{HbR}$, and $\mathrm{HbT}$ during the Word Expression Paradigm.

Table 7: A table of the mean values for each of the three stimuli across all 4 regions for $\mathrm{HbO}$, $\mathrm{HbR}$, and $\mathrm{HbT}$ during the Word Expression Paradigm. The red font indicates the region which recorded the largest amplitude of change for both the left and right hemisphere.

Table A1. A list of various research performed by other groups pertaining to language activation in the brain using both NIRS as well as various other modalities.

Table A2. A list of various research perfomed by other groups pertaining to functional connectivity and lateralization in the brain using both NIRS as well as various other modalities.

Table C1. A table showing the results of the t-test for the Word Expression Paradigm when comparing the 15 second average vs. the 30 second average for 2 randomly selected channels in the left and right hemispheres for $\mathrm{HbO}$, across all three stimuli. The 15 second average was selected from time 5 second through time 20 second. P values greater than 0.05 should not be considered statistically different.

Table C2. A table showing the results of the t-test for the Word Expression Paradigm when comparing the 15 second average vs. the 30 second average for 2 randomly selected channels in the left and right hemispheres for HbR, across all three stimuli. The 15 second average was selected from time 5 second through time 20 second. P values greater than 0.05 should not be considered statistically different. 
Table C3. A table showing the results of the t-test for the Word Expression Paradigm when comparing the 15 second average vs. the 30 second average for 2 randomly selected channels in the left and right hemispheres for HbT, across all three stimuli. The 15 second average was selected from time 5 second through time 20 second. P values greater than 0.05 should not be considered statistically different.

Table C4. A table showing the results of the t-test for the Word Reception Paradigm when comparing the 15 second average vs. the 30 second average for 2 randomly selected channels in the left and right hemispheres for $\mathrm{HbO}$, across all three stimuli. The 15 second average was selected from time 5 second through time 20 second. P values greater than 0.05 should not be considered statistically different.

Table C5. A table showing the results of the t-test for the Word Reception Paradigm when comparing the 15 second average vs. the 30 second average for 2 randomly selected channels in the left and right hemispheres for HbR, across all three stimuli. The 15 second average was selected from time 5 second through time 20 second. P values greater than 0.05 should not be considered statistically different.

Table C6. A table showing the results of the t-test for the Word Reception Paradigm when comparing the 15 second average vs. the 30 second average for 2 randomly selected channels in the left and right hemispheres for $\mathrm{HbT}$, across all three stimuli. The 15 second average was selected from time 5 second through time 20 second. P values greater than 0.05 should not be considered statistically different.

Table D1. A table of the mean and standard deviation of the 4 channel regional average and the 3 channel regional average for $\mathrm{HbO}$ during the Word Expression Paradigm.

Table D2. A table of the mean and standard deviation of the 4 channel regional average and the 3 channel regional average for HbR during the Word Expression Paradigm.

Table D3. A table of the mean and standard deviation of the 4 channel regional average and the 3 channel regional average for HbT during the Word Expression Paradigm.

Table D4. A table of the mean and standard deviation of the 4 channel regional average and the 3 channel regional average for $\mathrm{HbO}$ during the Word Reception Paradigm.

Table D5. A table of the mean and standard deviation of the 4 channel regional average and the 3 channel regional average for HbR during the Word Reception Paradigm.

Table D6. A table of the mean and standard deviation of the 4 channel regional average and the 3 channel regional average for HbT during the Word Reception Paradigm. 
Table D7. A table showing the results of the t-test for the Word Expression Paradigm when comparing each individual channel to the overall regional average for each stimuli ( FA-Forward Audio, RA- Reverse Audio, R-Rest) for HbO. P values greater than 0.05 should not be considered statistically different.

Table D8. A table showing the results of the t-test for the Word Expression Paradigm when comparing each individual channel to the overall regional average for each stimuli ( W.G-Word Expression, J.M- Jaw Movement, R-Rest) for HbR. P values greater than 0.05 should not be considered statistically different.

Table D9. A table showing the results of the t-test for the Word Expression Paradigm when comparing each individual channel to the overall regional average for each stimuli ( W.G-Word Expression, J.M- Jaw Movement, R-Rest) for HbT. P values greater than 0.05 should not be considered statistically different.

Table D10. A table showing the results of the t-test for the Word Reception Paradigm when comparing each individual channel to the overall regional average for each stimuli ( FA-Forward Audio, RA- Reverse Audio, R-Rest) for HbO. P values greater than 0.05 should not be considered statistically different. 109

Table D11. A table showing the results of the t-test for the Word Reception Paradigm when comparing each individual channel to the overall regional average for each stimuli ( FA-Forward Audio, RA- Reverse Audio, R-Rest) for HbR. P values greater than 0.05 should not be considered statistically different.

Table D12. A table showing the results of the t-test for the Word Reception Paradigm when comparing each individual channel to the overall regional average for each stimuli ( FA-Forward Audio, RA- Reverse Audio, R-Rest) for HbT. P values greater than 0.05 should not be considered statistically different.

Table D13. A table showing the results of the t-test for the Word Expression Paradigm when comparing each individual channel to the overall regional average for each stimuli ( W.G-Word generation, J.M-Jaw Movement, R-Rest) for HbO. P values greater than 0.05 should not be considered statistically different.

Table D14. A table showing the results of the t-test for the Word Expression Paradigm when comparing each individual channel to the overall regional average for each stimuli ( W.G-Word generation, J.M-Jaw Movement, R-Rest) for HbR. P values greater than 0.05 should not be considered statistically different.

Table D15. A table showing the results of the t-test for the Word Expression Paradigm when comparing each individual channel to the overall regional average for each stimuli ( W.G-Word generation, J.M-Jaw Movement, R-Rest) for HbR. P values greater than 0.05 should not be considered statistically different. 
Table D16. A table showing the results of the t-test for the Word Reception Paradigm when comparing each individual channel to the overall regional average for each stimuli ( FA-Forward Audio, RA- Reverse Audio, R-Rest) for HbO. P values greater than 0.05 should not be considered statistically different.

Table D17. A table showing the results of the t-test for the Word Reception Paradigm when comparing each individual channel to the overall regional average for each stimuli ( FA-Forward Audio, RA- Reverse Audio, R-Rest) for HbR. P values greater than 0.05 should not be considered statistically different.

Table D18. A table showing the results of the t-test for the Word Reception Paradigm when comparing each individual channel to the overall regional average for each stimuli ( FA-Forward Audio, RA- Reverse Audio, R-Rest) for HbT. P values greater than 0.05 should not be considered statistically different.

Table E1: A table of the three paired p-test results for Oxy-hemoglobin $(\mathrm{HbO})$ during the Word Expression Paradigm.

Table E2: A table of the three paired p-test results for Deoxy-hemoglobin (HbR) during the Word Expression Paradigm.

Table E3 :A table of three paired p-test results for Total hemoglobin (HbT) during the Word Expression Paradigm.

Table E4: A table of the three paired p-test results for Oxy-hemoglobin $(\mathrm{HbO})$ during the Regional Word Expression Paradigm.

Table E5: A table of the three paired p-test results for Deoxy-hemoglobin (HbR) during the Regional Word Expression Paradigm.

Table E6: A table of the three paired p-test results for Total hemoglobin $(\mathrm{HbT})$ during the Regional Word Expression Paradigm.

Table F1: A table of the three paired p-test results for Oxy-hemoglobin ( $\mathrm{HbO})$ during the Word Reception Paradigm.

Table F2: A table of the three paired p-test results for Deoxy-hemoglobin ( $\mathrm{HbR})$ during the Word Reception Paradigm.

Table F3: A table of the three paired p-test results for Total hemoglobin ( $\mathrm{HbT})$ during the Word Reception Paradigm.

Table F4: A table of the three paired p-test results for Oxy-hemoglobin $(\mathrm{HbO})$ during the regional Word Reception Paradigm. 
Table F5: A table of the three paired p-test results for Deoxy-hemoglobin (HbR) during the regional Word Reception Paradigm.

Table F6: A table of the three paired p-test results for Total hemoglobin (HbT) during the regional Word Reception Paradigm. 


\section{Acronyms}

AC- Alternating Current

ANOVA-Analysis of Variance

CW- Continuous Wave

DC- Direct Current

EEG- Electroencephalography

EMG- Electromyography

F.A- Forward Audio

FD- Frequency Domain

fMRI- Functional Magnetic Resonance Imaging

GUI- Graphical User Interface

HbO- Oxy-Hemoglobin

HbR- Deoxy-Hemoglobin

HbT- Total Hemoglobin

HomER- Hemodynamic Evoked Response

J.M- Jaw Movement

LA- Left Anterior

LP- Left Posterior

NIRS- Near Infrared Spectroscopy

PET- Positron Emission Spectroscopy

PMT- Photo multiplier tube

OD- Optical Density

R- Rest

R.A- Reverse Audio

RA- Right Anterior 
RP- Right Posterior

TD- Time Domain

W.G- Word Generation 


\section{Chapter 1-Introduction}

Near infrared spectroscopy (NIRS) is a non-invasive, non-ionizing imaging technique that offers the ability to perform real time studies in human subjects in the brain. This technique has been applied to many different areas of the brain since it was first implemented by Jobsis, which are explained in more detail in the background. ${ }^{[1]}$ As previously described, NIRS offers a therapeutic window which allows for the separation and quantification of both oxy-hemoglobin, and deoxy-hemoglobin. These two biological markers have been associated with brain activity, when the brain has been presented a given stimuli. Performing these types of studies in the brain in real time requires the use of a near infrared spectroscopy instrument. To date, there are numerous NIRS systems that are commercially available. ${ }^{[1,2]}$ For our studies a frequency domain based NIRS system was implemented (see section 3.1). Past language studies performed in the brain have looked into several different aspects of brain function pertaining to language, whether it be the activation of one of the two language regions, or the connectivity, or lateralization. However, no group to date, have reported the activation, functional connectivity, and lateralization to see if there is any correlation amongst these three aspects of brain function, in both language areas of the brain ( Broca's and Wernicke's region) using NIRS.

The objective of this work is to understand in detail how language functions in the temporal region of the brain using NIRS, which encompass the two language areas of the brain (i.e. Broca's and Wernicke's region). Past NIRS studies have only looked at activation, functional connectivity, or lateralization, in isolation to assess language function in the brain. This study looks to perform not only activation analysis, but also into the synchrony between the 
left and right hemispheres of the language regions (i.e. functional connectivity), as well as the dominant hemisphere (i.e. cortical lateralization). Once these aspects have been measured, they will be compared to see if there is any correlation amongst activation, functional connectivity, and lateralization, during a language task in the temporal region of the brain. By understanding and correlating activation, connectivity, and lateralization together, a better understanding of language function in the brain may evolve.

Once this study has been performed in a normal healthy population, the same study will further be applied to a population affected by a disease (in the future). In our case, the future direction of the current work is to apply this language based NIRS study to epileptic populations. Language is a useful tool when dealing with epileptic populations, since many of these subjects have surgery in different areas of the brain, where removal of brain tissue affected by the epileptic foci is vital in improving the patient's quality of life. The drawback to these types of procedures is sometimes the surgery may remove too much tissue, which may in turn affect other fully functioning areas of a subject's brain. The long term goal of our NIRS study is to provide a better understanding of the language dominance and connectivity's in the brain, such that removal of those regions during surgery may be avoided.

\subsection{Methodology}

The specific tasks carried out towards the current research are as described below:

\section{Specific Task \#1}

Perform a Word Expression paradigm using NIRS on 15 normal adults, and measuring the activation, functional connectivity, and lateralization (both channel-wise and region-wise), as well as any correlations amongst them. 
Specific Task \#2

Perform a Word reception paradigm using NIRS on 15 normal adults, and measuring the activation, functional connectivity, and lateralization (both channel-wise and region-wise), as well as any correlations amongst them.

\subsection{Thesis Layout}

The background of NIRS, its theory in brain mapping as well as NIRS imaging techniques and applications of NIRS and work done to date using NIRSare described in the background chapter (Chapter 2). Using an NIRS based optical imaging system, which will be described in complete detail in the materials and methods section of Chapter 3 (section 3.1), both a Word Expression and Word Reception paradigms were performed on 15 right-handed normal adult subjects. The details of both the paradigms the entire experimental study and the pertinent statistical and data analysis procedures are described in Chapter 3 as well. The activation, functional connectivity, and lateralization results of both the paradigms are described as two separate results chapters (Chapters 4 and 5, respectively), followed by conclusion and future direction of the current work in Chapter 6.

Chapter 2-Background

2.1-NIRS Overview and Theory 
Near infrared spectroscopy (NIRS) is a technique that relies on light in the range of $600 \mathrm{~nm}-900 \mathrm{~nm}$. This range is known as the "therapeutic window" (see Figure 1B). Light in this region is minimally absorbed, and preferentially scattered, allowing for separate signals to be obtained at multiple wavelengths. As each wavelength of light propagates through the tissue, there is an exponential decay with respect to the initial intensity of light launched at the surface of the tissue. As light propagates through the various tissues it encounters in the body, the light becomes either absorbed or scattered. These events cause the light to lose intensity, and the detected light seen by the photon detector has less intensity than the initial intensity the light was launched with. Once each wavelength of light has been collected by photon detectors at the tissue surface, separation of light based on specific wavelength allows for the back-calculation of the relative concentrations of both oxy and deoxy hemoglobin. Due to the low absorption of light by the body in this range, the depth of penetration can be as much as a few centimeters (see figure 1a).

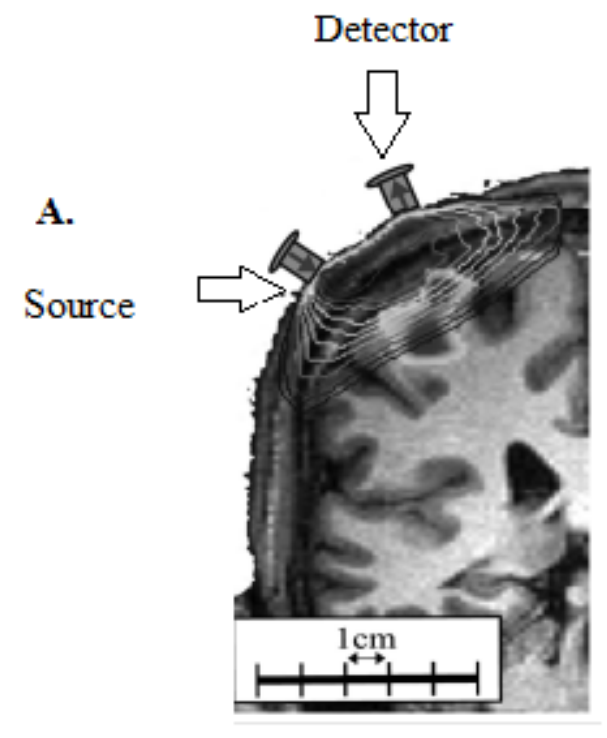

B. Therapuetic Window

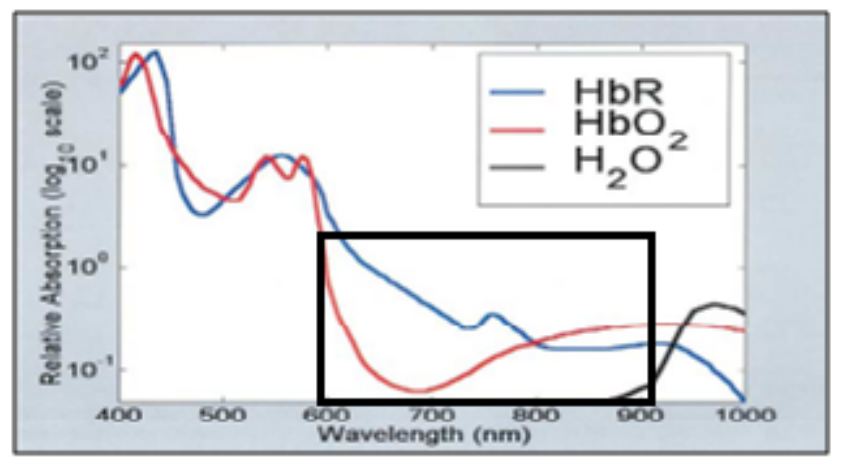


Figure 1. (A) Sensitivity of NIRS instrumentation, showing light propagating from a laser source to a detector several centimeters away, (B ) “Therapeutic window," Absorption spectrum of oxyhemoglobin and deoxyhemoglobin.

\section{2- NIRS Imaging Techniques}

Due to the growth of the NIRS field, there are now an abundance of commercially available optical instrumentation systems: Imagent ISS, NIRO 500, Hamamatsu, and Hitachi, to name a few. NIRS also offers several different techniques of performing experiments: (1) Continuous wave, (2) Frequency domain, (3) Time domain. In the continuous wave technique, a stable light source continuously propagates light into a given tissue. The light is then collected once it has propagated through the tissue, and the intensity of the collected light is used to determine the amount of optical absorption and scattering by the tissue. The frequency domain technique utilizes modulated light signals, and measures the output intensity of light and the phase shift to determine the optical properties of the tissue of interest. Time domain uses very fast lasers to send pico-second pulses into the tissue of interest, and the temporal distribution of photons are collected and recorded once the light passes through the tissue, as well as the intensity of light that has propagated through the tissue along with the time lag of photons entering the detector.

The time domain (TD) technique relies on gating late photons, which are collected based on the time of arrival of light incident on the detector. ${ }^{[4]}$ By using the TD technique, better depth sensitivity can be provided than that of the $\mathrm{CW}$ technique. The time domain technique can provide better depth sensitivity due to the fact that late arriving photons carry information about 
deeper layers of the brain, and by rejecting early photon arrivals, which is sensitive to superficial physiological signal clutter. ${ }^{[4]}$

Frequency domain technique can also be used to measure cerebral hemodynamic changes. This is due to the fact that frequency and time information are related through the Fourier transform. ${ }^{[4]}$ In the frequency domain technique, the light source is intensity modulated through the use of radio frequencies. Measurements are not based solely on the detected light intensity, but also the phase shift and modulation depth with respect to the input light intensity. ${ }^{[3,4]}$ Previous groups have demonstrated that for typical biological tissue, with a modulation frequency of less than $200 \mathrm{MHz}$, the phase shift is linearly related to the average path length of light. ${ }^{[5,6]}$ The frequency domain technique is a low cost alternative to pulse based methods of time resolved imaging. ${ }^{[7]}$ While the time domain and the frequency domain techniques are both utilized in brain imaging studies, the most often used technique is still the continuous wave $(\mathrm{CW})$ approach, due to its ability to provide higher temporal resolution, as well as the lower cost of photon detectors, which provide a greater number of spatially resolved measurements. ${ }^{[3]}$

\section{3- Continuous Wave NIRS Theory}

In order to apply the technique of NIRS to brain imaging, an array of laser sources are created and placed on the region of interest, or on the entire head. The placement of light sources usually relies on the international 10-20 EEG placement system. As previously mentioned, NIRS is minimally absorbed by the body, which allows for sampling of the outer 1.5$2 \mathrm{~cm}$ of the head through the skin and skull, to reach the outer $5-10 \mathrm{~mm}$ of brain tissue, approximately. ${ }^{[3]}$ Light sources emit light into the skull, where the photons become highly 
scattered, and usually exit the skull $3-4 \mathrm{~cm}$ away from the light source. By placing detectors roughly $3 \mathrm{~cm}$ away, the light signal being passed through the head can be collected over a diffuse volume. ${ }^{[3]}$

In the brain, changes in the optical absorption are related to the changes in both oxyhemoglobin and deoxy-hemoglobin. Both the relative concentration of oxy and deoxyhemoglobin can be estimated from the optical measurements of the changes in the absorption at multiple wavelengths. This is due to the unique absorption signatures of the oxy- and deoxyhemoglobin. The changes in relative concentrations of both hemoglobin species in the brain are usually in response to a given activation or stimulation task, and are known as functionally evoked responses.

As previously mentioned, there are several ways to perform NIRS imaging, these include: (i) Continuous wave imaging, (ii) Frequency domain imaging, and (iii) time domain imaging. The discussion here will be limited to continuous wave imaging, since this was the approach used to attain the data described in the current study. Continuous wave imaging $(\mathrm{CW})$ uses a continuous light intensity source to propagate a beam of light into the tissue of interest. Once the light propagates through the tissue, a detector measures the intensity of light that propagated through the tissue to determine the amount of optical absorption by the sample (tissue). By using multiple light sources, and photon detectors, arranged in a grid on the subjects head, noninvasive measurements of the optical measurements of the absorption changes in the tissue volume between source- detector pairs can be recorded.

Using diffusion approximations or Monte-Carlo based computer simulations, ${ }^{[3,8,9,10,11-14]}$ the sensitivity profile of all the source-detector pairs can be estimated on the basis of the optical 
properties of the tissue (illustrated in Fig (1a)). Light encounters several tissue layers before reaching the outer $5-8 \mathrm{~mm}$ of brain tissue. These layers include the scalp $(0.5-1 \mathrm{~cm})$, the skull ( $0.5-1 \mathrm{~cm})$, and cerebral spinal fluid layer $(0-2 \mathrm{~mm}) \cdot{ }^{[3]}$ Due to differences in the varying thicknesses of these layers of tissue, which are caused by gender, age, and other inter-subject factors, sensitivity of NIRS measurements to brain activity may be affected and can cause potential biases in the spatial localization of brain activity. ${ }^{[15]}$ This is a drawback in using noninvasive imaging, and it arises from the fact that light has to propagate through the tissue, and the thickness and composition of the tissue influences how the light propagates through the head, which varies from subject to subject.

When the brain becomes activated, increases in blood flow, volume, and oxygen metabolism all cause the oxygen saturation levels of the blood in the brain to rise and fall. ${ }^{[12]}$ As the amount of oxy-hemoglobin and deoxy-hemoglobin changes in the brain, the amount of light that is differentially absorbed due to these parameters also changes compared to the rest state (baseline). This change in light absorption is known as the change in optical density ( $\Delta \mathrm{OD})$, and can be calculated by normalizing the light changes incident on a detector from a given source position. The change in optical density $(\Delta \mathrm{OD})$ is defined as

$$
\begin{aligned}
\phi_{i j}^{\lambda}(t) & =\int \phi_{i j}^{\lambda}(0) e^{-\Delta \mu^{\lambda} a b s(\vec{r}) \circ d \vec{r}} \\
\Delta O D_{i j}^{\lambda}(t) & =\operatorname{Ln}\left[\frac{\phi_{i j}^{\lambda}}{\phi_{i j}^{\lambda}(t)}\right]
\end{aligned}
$$

where $\Phi$ (intensity) is the amount of light emitted by a source at position (i) that is incident on a detector at position $(j)$. The term $\Delta \mu_{\text {abs }}\left(\mathrm{cm}^{-1}\right)$ describes the change in the absorption coefficient compared to baseline levels (rest state). In equations (1a) and (1b), each time series recording of 
the light intensity from source position $(i)$ incident on detector position $(j)$ is represented by $(\mathrm{t})$ (sec.), and the wavelength used is represented by $\lambda(\mathrm{nm})$. The integral in Eqn. (1a) are functional path integrals over the volume sampled by the numerous possible photon trajectories through the tissue, which is defined by the stochastic diffusion of light in tissue. The amount of light intensity that is detected is proportional to the amplitude of voltage signal reported by the photon detectors, which assumes that the instability and possibility of detector nonlinearity of the detectors of the NIRS instrument used can be neglected. Detector efficiency, detector analog amplification (Gain), and the instruments absolute incident laser power can be negated by normalizing the intensity measurements to the incident light at the start of the experiment (usually done with the mean of the signal over the course of the study). These factors (detector efficiency, detector analog amplification (Gain), absolute incident laser power) can be negated due to normalizing since these factors appear as scaling factors that multiply both numerator and denominator, as seen in Eqn. (1b). This attribute is an important factor in CW NIRS imaging since it removes the need for calibration of the instrument, but the drawback to normalizing means that CW NIRS is restricted to providing information about the relative changes in hemodynamic evoked responses rather than absolute values of hemoglobin.

It is assumed for optical functional brain measurements that the absorption changes due to hemoglobin are small, and do not affect the path of light through the tissue. ${ }^{[1]}$ With this assumption, absorption changes can be expressed linearly, as a combination of the changes in both oxy-hemoglobin $(\mathrm{HbO})$ and deoxy-hemoglobin $(\mathrm{HbR})$. This is expressed mathematically by replacing the path integral in Eqn. (1a) with the multiplication by the effective mean path length term, which represents the average path of light traveled through the illuminated 
region. ${ }^{[16,17]}$ The relationship formed through the multiplication of Eqn. (1a) with the effective mean path length represents a linear relationship between the changes in optical density and the concentration of absorbing species in the sample, and is referred to as the Beer Lambert Law.

$$
\Delta O D_{i j}^{\lambda}=L_{i j}^{\lambda}\left(\varepsilon_{H b R}^{\lambda} \Delta[H b R]+\varepsilon_{H b o}^{\lambda} \Delta[H b O]\right) \quad \text { Eqn. (2) }
$$

where $\varepsilon\left(\mathrm{M}^{-1} \mathrm{~cm}^{-1}, \mathrm{M}=\right.$ molarity) represents the wavelength dependent extinction coefficient for each hemoglobin species. $L_{i j}(\mathrm{~cm})$ represents the mean path length traveled from the source position $i$ to the detector position $j$.

\section{4- Applications of NIRS}

In 1977, Jobsis ${ }^{[18]}$ was the first to demonstrate the technique of NIRS in measuring cerebral changes. Since Jobsis first demonstrated this technique, it has been applied in various regions of the brain including visual ${ }^{[19-21]}$, auditory ${ }^{[22-24]}$, and somatosensory cortices ${ }^{[25,26]}$, as well as in the motor cortex ${ }^{[27-30]}$, prefrontal cortex ${ }^{[31-34]}$, and in language areas of the brain. ${ }^{[35,36]}$

As previously mentioned, there have been numerous studies performed on the brain. These studies range from performing NIRS on the visual cortex of infants, to measuring motor cortex activation in response to a given stimuli. Other applications include studying visual cortex, auditory cortex, the somato-sensory cortices, and the prefrontal region of the brain. Understanding of the brains function in response to language has been a topic of major interest. In the brain there are two major areas located in the temporal region which are responsible for language. The first area, known as Broca's region, is responsible for language expression. The second region, known as Wernicke's region, is responsible for language reception. NIRS has been applied with success in monitoring the brain to understand the function of language. Cannestra et. al (2003) used NIRS to study the activation in Broca's area in response to 
language stimuli. ${ }^{[37]}$ More recently Ehlis et.al. (2007) showed activation in both prefrontal and temporal regions of the brain in response to language stimuli in both healthy control subjects, and schizophrenic patients. ${ }^{[38]}$ Quaresima et. al (2002). have demonstrated activation in response to language translation tasks specifically in the left inferior cortex (encompassing the Broca's area). ${ }^{[39]}$ Brauer et. al. (2007) performed a study in the perisylvian region, which encompasses both language areas, in which they compared activation induced by language processing tasks in both children and adults, finding differences in the activations between the two groups. ${ }^{[40]}$ Schecklmann et al. (2008) reported the activation as well as the correlation between activations in the temporal region as well as the prefrontal cortex in response to language generation stimuli.

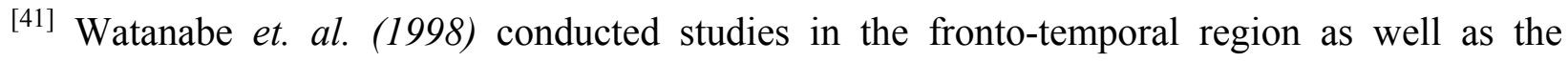
superior temporal region, including Broca's area, measuring the activation as well as the lateralization in these regions. ${ }^{[42]}$ While numerous groups have reported activation, functional connectivity, and/or lateralization (in isolation) for a language-based temporal brain study, no study to date correlates these different aspects of brain mapping. Furthermore, many groups have only looked at either Broca's or Wernicke's area in response to a single language paradigm, while other groups do not even look specifically at these language regions, but rather just the temporal cortex. It is important to understand the functionality of these two regions simultaneously under specific language paradigms, and to use not only activation as a method of measuring the brains activity, but to also correlate the functional connectivity and lateralization as well. By looking at these areas in greater depth, a better picture can be drawn as to how the two language areas of the brain function in response to language. A table below is given showing work done to date in both Broca's and Wernicke's area involving language related tasks, and their results. 
Table 1 below is shown to demonstrate some of the work done to date in the brain in response to language. There have been many groups to study language and brain function, both using NIRS and other imaging modalities (i.e. Functional Magnetic Resonance imaging (fMRI), Positron Emission Tomography(PET), Electromyography (EMG), etc.). ${ }^{[43-46,47,48,49]}$ The table demonstrates that while groups have done this work and reported either activation, functional connectivity, or lateralization, no group has looked at both Broca's and Wernicke's area simultaneously bilaterally, under two separate paradigms, and reported activation, functional connectivity, and lateralization, using NIRS. A separate table is provided in appendix A, table A2 to show some of the work done to date studying language in the brain using non NIRS modalities (i.e. fMRI, PET, EEG, EMG, etc.).

\begin{tabular}{|c|c|c|c|c|}
\hline Title & Author(s) & $\begin{array}{l}\text { Region of } \\
\text { Interest(ROI) }\end{array}$ & Stimuli & Results \\
\hline $\begin{array}{l}\text { "Multichannel near- } \\
\text { infrared spectroscopy } \\
\text { in depression \& } \\
\text { schizophrenia: } \\
\text { cognitive brain study" }\end{array}$ & Suto et. al (2004) & Frontal and Temporal Lobes & Word Fluency & $\begin{array}{l}\text { Time courses differed } \\
\text { between depressed \& } \\
\text { schizophrenic patients, } \\
\text { but marked increase in } \\
\text { HbO compared to control } \\
\text { group( healthy subjects) }\end{array}$ \\
\hline $\begin{array}{l}\text { "Assessing infants' } \\
\text { cortical response to } \\
\text { speech using NIRS" }\end{array}$ & $\begin{array}{l}\text { Bortfield, H., Wruck, E., } \\
\text { Boas,D.(2007) }\end{array}$ & $\begin{array}{l}\text { Left Temporal and Occipital } \\
\text { Regions }\end{array}$ & $\begin{array}{l}\text { Word reception tasks, as } \\
\text { well as visual stimulation }\end{array}$ & $\begin{array}{l}\text { Robust and reliable } \\
\text { patterns of activation in } \\
\text { left temporal region and } \\
\text { primary visual region of } \\
\text { neocortex, increases in } \\
\text { HbO in left temporal } \\
\text { region during speech } \\
\text { exposure }\end{array}$ \\
\hline $\begin{array}{l}\text { "Functional } \\
\text { assessment of Broca's } \\
\text { area using near } \\
\text { infrared spectroscopy } \\
\text { in humans" }\end{array}$ & $\begin{array}{l}\text { Cannestra, A.F, } \\
\text { Wartenburger, I., Obrig, } \\
\text { H.M., Villringer, A. } \\
\text { Toga, A.W(2003) }\end{array}$ & $\begin{array}{l}\text { Language and Motor } \\
\text { cortices of Broca's region }\end{array}$ & $\begin{array}{l}\text { Tongue movement, and } \\
\text { covert visual object } \\
\text { naming, as well as finger } \\
\text { tapping. }\end{array}$ & $\begin{array}{l}\text { HbR was more localized; } \\
\text { Broca's area was easily } \\
\text { distinguished from } \\
\text { neighboring tongue and } \\
\text { motor representation, } \\
\text { HbR preferential NIRS } \\
\text { marker to map language } \\
\text { cortices. }\end{array}$ \\
\hline $\begin{array}{l}\text { Functional near- } \\
\text { infrared spectroscopy: }\end{array}$ & Schecklmann et. & $\begin{array}{l}\text { Prefrontal and parts of } \\
\text { Superior temporal cortex }\end{array}$ & Word generation tasks & $\begin{array}{l}\text { No difference between } \\
\text { three stimuli w/ regard to }\end{array}$ \\
\hline
\end{tabular}




\begin{tabular}{|c|c|c|c|c|}
\hline $\begin{array}{l}\text { A long term reliable } \\
\text { tool for measuring } \\
\text { brain activity during } \\
\text { verbal fluency" }\end{array}$ & $a l(2008)^{[41]}$ & & & $\begin{array}{l}\text { fluency, did see weak } \\
\text { correlation using Pearson } \\
\text { corr. Test. }\end{array}$ \\
\hline $\begin{array}{l}\text { "Non-Invasive } \\
\text { assessment of } \\
\text { language dominance } \\
\text { w/NIRS mapping" }\end{array}$ & Watanabe et. al(1998) $)^{[42]}$ & $\begin{array}{l}\text { Fronto-temporal, and } \\
\text { inferior temporal gyrus, } \\
\text { including Broca's area }\end{array}$ & $\begin{array}{l}\text { Word generation task, } \\
\text { both oral (speaking) and } \\
\text { writing }\end{array}$ & $\begin{array}{l}\text { Healthy subject } \\
\text { dominance observed, } \\
\text { agreed with expected } \\
\text { given the handedness of } \\
\text { subject. Handedness may } \\
\text { not affect cerebral } \\
\text { (hemispheric } \\
\text { dominance). }\end{array}$ \\
\hline $\begin{array}{l}\text { "Cortical activation } \\
\text { during two verbal } \\
\text { fluency tasks in } \\
\text { schizophrenic patients } \\
\text { and healthy controls } \\
\text { as assessed by } \\
\text { multichannel NIRS" }\end{array}$ & $\begin{array}{l}\text { Ellis, A.C., Herrmann, M.J., } \\
\text { Plichta, M. M., Fallgatter, } \\
\text { A.J. }(2007)^{[38]}\end{array}$ & $\begin{array}{l}\text { Frontal Lobe, Fronto- } \\
\text { temporal lobe, Temporal } \\
\text { lobe }\end{array}$ & $\begin{array}{l}\text { Word Generation tasks( } \\
\text { both word naming, and } \\
\text { category naming) }\end{array}$ & $\begin{array}{l}\text { Letter version induced } \\
\text { stronger activation than } \\
\text { category, differences } \\
\text { from phonological } \\
\text { fluency, positive } \\
\text { correlation between task } \\
\text { related activation in both } \\
\text { prefrontal and temporal }\end{array}$ \\
\hline $\begin{array}{l}\text { "Lateral frontal cortex } \\
\text { oxygenation changes } \\
\text { during translation and } \\
\text { language switching } \\
\text { revealed by non } \\
\text { invasive near infrared } \\
\text { multipoint } \\
\text { measurements" }\end{array}$ & $\begin{array}{l}\text { Quaresima, V., Ferrari,M., } \\
\text { van der sluiis, C.P, } \\
\text { Menssen, J., Colier, } \\
\text { W.N.J.M (2002) }\end{array}$ & $\begin{array}{l}\text { Left lateral frontal lobe, } \\
\text { temporal lobe, Broca's area }\end{array}$ & $\begin{array}{l}\text { Asked to translate } \\
\text { language from Native } \\
\text { tongue(L1) to English( L2) } \\
\text { both verbal and written }\end{array}$ & $\begin{array}{l}\text { Evoked } \mathrm{HbO} \text { increase in } \\
\text { left inferior frontal cortex } \\
\text { (including Broca's) } \\
\text { followed as well by small } \\
\text { decrease in HbR, areas } \\
\text { around broca showed no } \\
\text { consistent or uniform } \\
\text { concentration changes to } \\
\text { activation task, Brocas } \\
\text { area is involved in } \\
\text { translational process and } \\
\text { activation is unaffected } \\
\text { by direction of translation }\end{array}$ \\
\hline $\begin{array}{l}\text { "Multi-channel near } \\
\text { infrared spectroscopy } \\
\text { detects specific } \\
\text { interior frontal } \\
\text { activation during } \\
\text { incongruent Stroop } \\
\text { trials" }\end{array}$ & Ehlis et. al(2005) ${ }^{[51]}$ & Lateral Frontal, Temporal & $\begin{array}{l}\text { Stroop color word tasks, } \\
\text { word generation tasks }\end{array}$ & $\begin{array}{l}\text { Similar activation } \\
\text { patterns for } \\
\text { congruent/incongruent } \\
\text { stroop, in superior } \\
\text { frontal. Marked increase } \\
\text { in HbO in this area } \\
\text { indicates specific } \\
\text { activation for } \\
\text { interference trials in } \\
\text { inferior frontal areas of } \\
\text { left hemisphere. }\end{array}$ \\
\hline
\end{tabular}

Table 1: Work done to date on language function in the brain using NIRS.

NIRS is an invaluable tool that has shown its potential in the application of brain imaging. Previous groups have shown that it provides a good spatial and temporal resolution, with the ability of detecting relative changes of both oxy and deoxy-hemoglobin in the brain, in response to various stimuli. ${ }^{[1,3,42,52]}$ To date there have been numerous studies that have looked into the activation, or functional connectivity, or lateralization, in various parts of the brain using both NIRS and other modalities (Table 1, and Tables A1 and A2 of Appendix A). No group to 
date has correlated these three aspects in the temporal region of the brain (which encompass both areas in the brain responsible for language) in response to two specific language paradigms using NIRS. The objective of this study is to perform two separate language based paradigms on normal healthy adults in the temporal region of the brain, and correlating the activation, functional connectivity, and lateralization using NIRS.

The purpose of correlating these aspects is to try to provide a better understanding of how the brain functions in response to language, by giving a more detailed analysis of brain function itself (i.e. how it activates, which areas are correlated, what neuronal pathways are used, and which hemispheres are dominant). The next chapter provides details into the experimental approach, and all the steps taken to accomplish both studies.

\section{Chapter 3-Materials \& Methods}

In order to perform two separate language paradigms on an adult population, certain experimental procedures were necessary. In the following chapter, the instrumentation used in

both experiments is described, as well as the head gear used to hold both sources and fibers in their correct location. Furthermore, the placement system (International EEG 10-20 placement system) is described in detail. Both experimental paradigms used in this experiment are also described (language expression, language reception), as well as the population sampled. The data analyses as well as statistical analyses performed on the results are also described in detail at the end of the chapter, along with the software used to perform both data analysis and statistical analysis. 


\section{1-Instrumentation}

An NIRS based optical imaging system (Imagent ISS Inc., Champaign IL.) (see Figure 2) with 32 sources and four detectors was used to conduct the study. The Imagent system used utilizes four photo-multiplier tube detectors, which offer the ability to detect light signals at a high acquisition rate $(<\mathrm{ms})$. In addition, the Imagent delivers light by using fiber optics, and utilizes frequency domain technology. In addition, Imagent also comes with a computer software known as $\mathrm{BOXY} \mathrm{C}^{\odot}$, which provides information to the user about the optimization of the system. $\mathrm{BOXY}^{\odot}$ provides real-time information regarding signal to noise ratios of the four photomultiplier tubes used by Imagent. When the signal to noise ratio falls below a certain threshold, the BOXY graphical user interface (GUI) indicates which detector fell below the said threshold, and how much light is now being received by the detector. The GUI indicates three separate threshold values, green indicates there is a high signal to noise ratio, yellow indicates that the signal to noise ratio is even, and red indicates that there is a high amount of noise with very minimal signal. For the studies performed in this thesis, any data that was collected with a signal indication other than green was discarded due to poor signal to noise ratio. The Imagent ${ }^{\mathrm{TM}}$ system offers the ability to modulate the sources at high frequencies (for this study frequency= $110 \mathrm{MHz}$ ) allowing for the measurement of three parameters of the detected signal (Averaged Intensity (DC), Intensity (AC), and phase shift). Since Imagent can operate in frequency domain it can provide measurements in either a DC signal or an AC signal. 


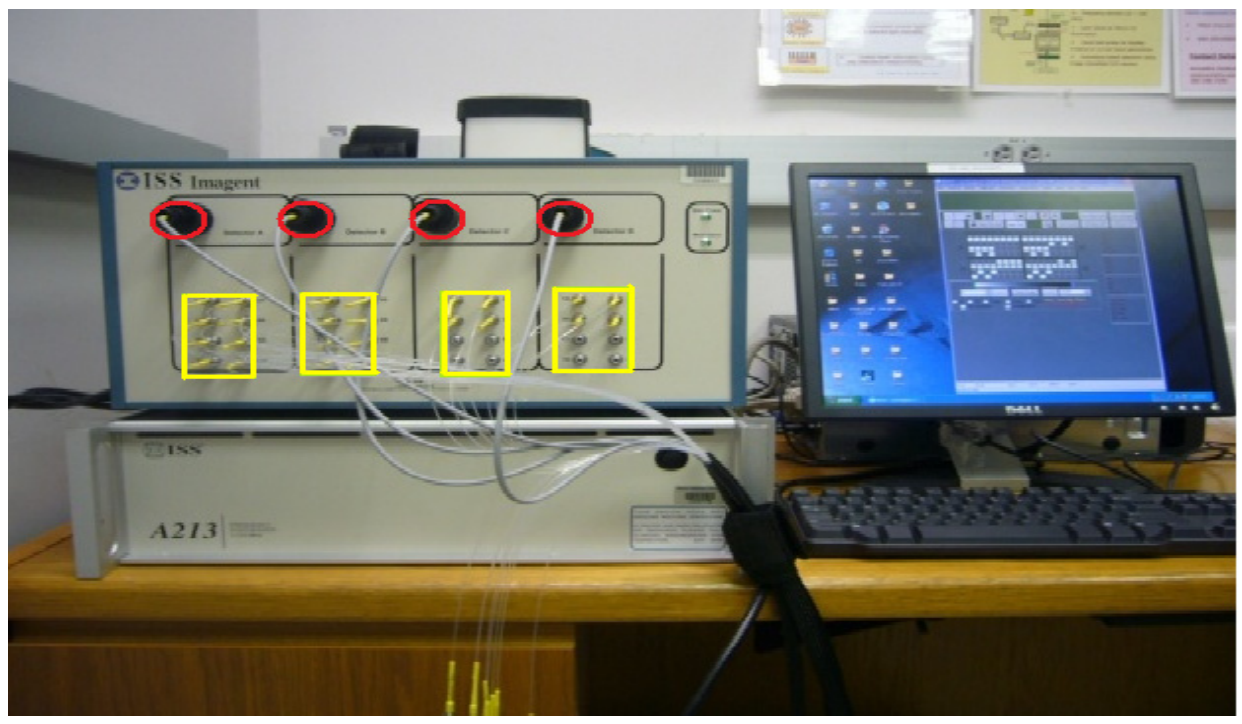

Figure 2. Frequency domain based optical imager, Imagent (ISS, Inc., Champaign, IL). The Imagent was provided by Brain Institute at MCH (Miami Children's Hospital).

For this study the DC signal was chosen since it is the average of the AC signal, and since it can also provide information regarding the relative concentration change in both $\mathrm{HbO}$ and $\mathrm{HbR}$. Fourteen sources and 4 detectors were used, with light being sent at two wavelengths, $690 \mathrm{~nm}$ and $830 \mathrm{~nm}$. The wavelengths were chosen based on the minimal absorption of light by each of the two chromophores of interest, $\mathrm{HbO}$, and $\mathrm{HbR}$. Since each chromophore has a different absorption coefficient at each of the two wavelengths, the relative concentration of both $\mathrm{HbO}$ and $\mathrm{HbR}$ can be calculated. Since this study was conducted in an adult population, sourcedetector separations were $3 \mathrm{~cm}$ for all source detector combinations (chosen such that the detected diffuse optical signals are from the cortical surface and not from the skull). ${ }^{[1,2]}$

The number of sources were chosen so that the entire perisylvian region (Broca's area and Wernicke's area) would be encompassed the maximum number of sources possible, while still maintaining correct source detector separation. The Imagent system is limited in the number 
of photon detectors (4 total photo-multiplier tubes). For the current study, two detectors were placed in the left temporal hemisphere, and two in the right hemisphere, with each detector being surrounded by 4 sources.

In order to properly ensure that a secure contact would be maintained for both sources and detectors with the scalp, and source detector distances would be constant, a custom-built optical head cap was utilized to place the source and detectors on to the temporal regions of the brain.

The cap containing source and detector fibers were in turn covered by a light proof black material to minimize ambient light interference. As previously mentioned, placement of the cap was in the perislyvian region of the brain, with the exact locations of the sources and detectors being dictated by the EEG international 10-20 system. This system is the standard placement system used worldwide in placing EEG optodes to the scalp. The EEG international 10-20 system was utilized in this study to ensure correct placement locations over both Broca's and Wernicke's area. A schematic of the source and detector geometry as well as the placement locations based on the EEG 10-20 system can be seen in Figure 3. 

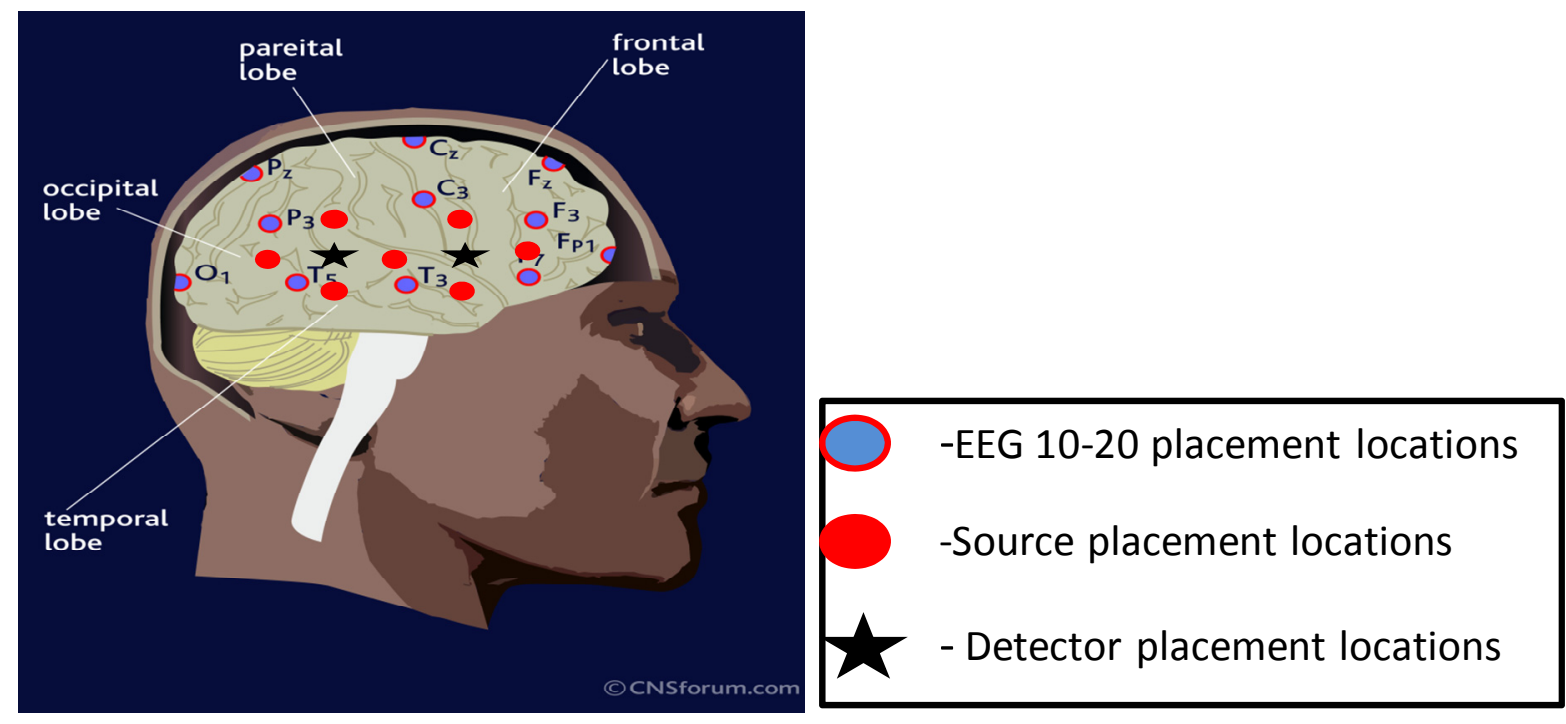

Figure 3. Source detector layout and Source detector placement based on International EEG 10-20 placement.

\section{2 - Participants}

Fifteen healthy right handed adults (between 18-30 years of age) were recruited to participate in the study. Right handedness was not tested, but based on which hand the participants predominately use. The study was Florida International University-Institutional Review Board (FIU-IRB) approved.. Each subject consented to the study, with all subject studies being carried out at Miami Children's Hospital (Miami,FL).

\section{3- Experimental Paradigm}

For this study, two different paradigms were presented: (i) a language generation based paradigm (word expression paradigm), and (ii) a language reception based paradigm (word reception paradigm) (as shown in Figure 4). There are two types of paradigms that are typically chosen when conducting studies in the brain, an event-related paradigm, and a block design paradigm. A block design was chosen for this experiment, rather than an event-related paradigm, 
due to the fact that this study is aimed at looking at detecting changes in the relative concentration of $\mathrm{HbO}$ and $\mathrm{HbR}$ over a given period of time in response to language stimuli. Both paradigms followed a block based experimental design, with a total of 5 blocks in each paradigm, and each paradigm being repeated twice (i.e. two replicates) for each subject. Both paradigms were designed with the help of Dr. Gustavo Rey, a neurophysiologist at Miami Children's Hospital.
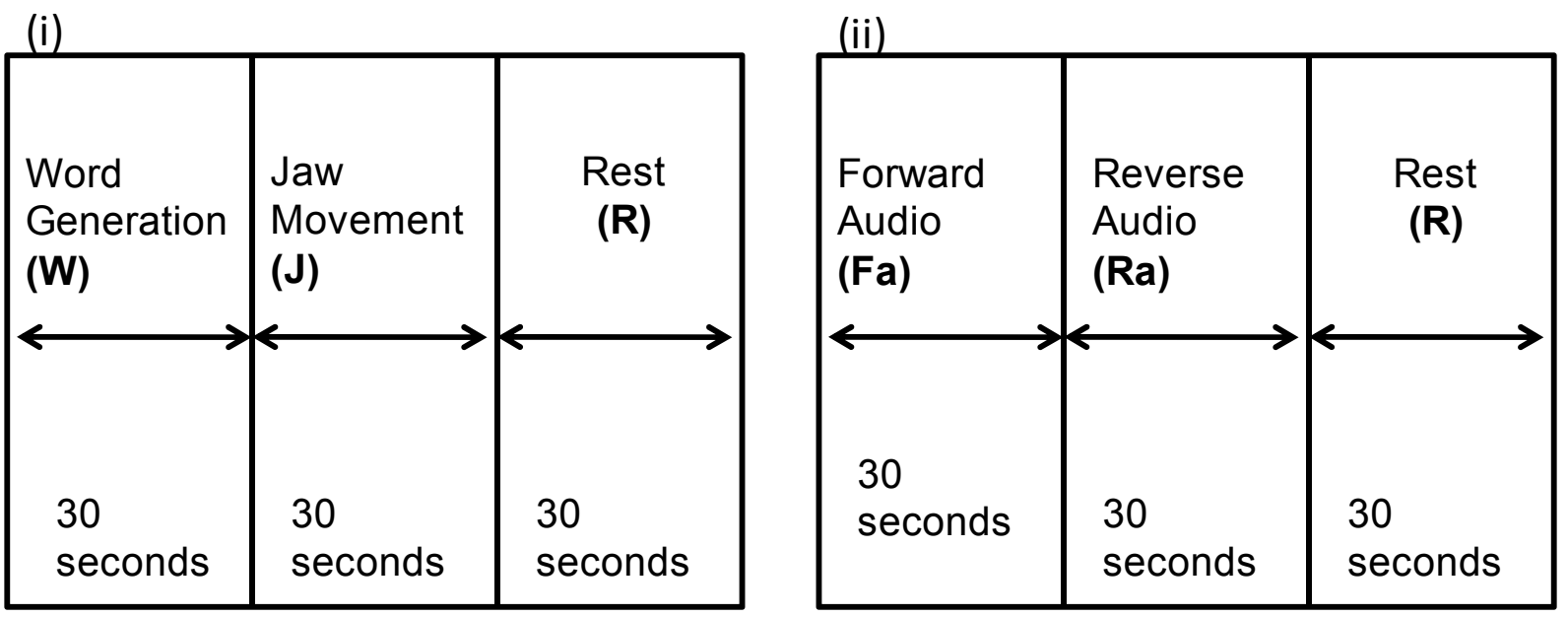

Figure 4: A diagram of the (i) Word Expression Paradigm, and (ii) Word Reception Paradigm.

\subsubsection{Word Expression Paradigm}

A five block experimental design was developed, with each block consisting of three 30second stimulus. The three stimuli include Word generation (W.G), Jaw movement (J.M), and 
Rest (R) conditions. During Word generation, subjects were asked to speak out as many words possible using a specific alphabet given by the presenter. The same set of letters was used in each of the two repeated studies among all subjects. During Jaw movement stimulus, the subjects were asked to move their jaws randomly as though they spoke without actual speaking. This stimulus was used as a baseline to differentiate (if any) between the hemodynamic responses from brain activation in response to a language-based stimuli and random jaw movement during speaking. Finally, the subjects were asked to rest for 30 -seconds, when they were asked to look at the blank wall facing then and clear their minds.

\subsubsection{Word Reception Paradigm}

A five block experimental design was utilized for this study. Each block consisted of three 30-second stimuli, which include Forward audio (F.A), Reverse audio (R.A), and Rest (R) conditions. In this paradigm subjects were asked to listen to two separate audio files. Each subject listened to the same audio files for a given replicate, in order to maintain consistency. The passages heard by the subjects were all rated at a $6^{\text {th }}$ grade reading level, taken from the Florida Comprehensive Assessment Test (FCAT) (http://www.fcat.fldoe.org) for $6^{\text {th }}$ graders. All of the audio files used in this study were also modulated such that they were read at the same frequency, using a free online text to speech software, known as Natural Soft $^{\odot}$, (http://www.naturalreaders.com/index.htm ) in order to minimize any variability. Additionally, all subjects heard the files at the same volume through a set of headphones. During Forward audio stimulus, the subjects heard a reading passage that was read to them by a computer reader, with a woman's voice. During Reverse audio stimulus, readers heard the same passage as in Forward audio, but in the reverse order (from the last word to the first word). The reverse audio 
task was designed to be a comparison task to the forward audio task. In the forward audio task subjects were able to comprehend everything being read to them. The reverse audio task incorporated to see how the brain functioned when the subject was unable to comprehend any language being read to them, to compare results to those where a subject is able to understand everything, to see if there is any difference in the response of the brain. Finally, the subjects were asked to rest for 30-seconds, when they were asked to look at the blank wall facing them and clear their minds.

\subsection{Data Analysis}

Data analysis was carried out to understand the brain activation, functional connectivity, and cortical lateralization in the temporal brain region in response to the two language-based paradigms.

Before any post processing analysis can take place, the raw optical signal collected was transformed into the evoked hemodynamic response. The raw optical signal that was collected was light that has been attenuated after passing through cerebral tissue.

As the light enters the cerebral tissue, it is attenuated by two dominant chromophores (oxy-hemoglobin (HbO), and deoxy-hemoglobin (HbR)). The amount of light that exits the tissue is a lower percentage of light than was originally launched into the tissue and the amount of attenuation is a function of the amount present of these two chromophores in the tissue at the time the light was launched.

In order to calculate the change in the optical density, which will be described in further detail below, the raw optical signal must first be processed. The theory of how the optical density 
is derived is given in detail Section 2.2. The steps given below are performed only after the reflected intensity from the tissue has been obtained. The following steps are used to convert the raw optical signal to tissue oxygenation:

Step 1: The raw optical data obtained is filtered using a bandpass filter $(0.3 \mathrm{~Hz}-0.0016 \mathrm{~Hz})$. This filtering is done to remove any physiologic noise, which can contaminate the data. Figure $5^{[3]}$ below shows physiological fluctuations in optical signals taken while recording a subject during a rest task. Table 2 below is also provided to show the time scale and frequency range for the different types of biological signals that can affect brain signals.

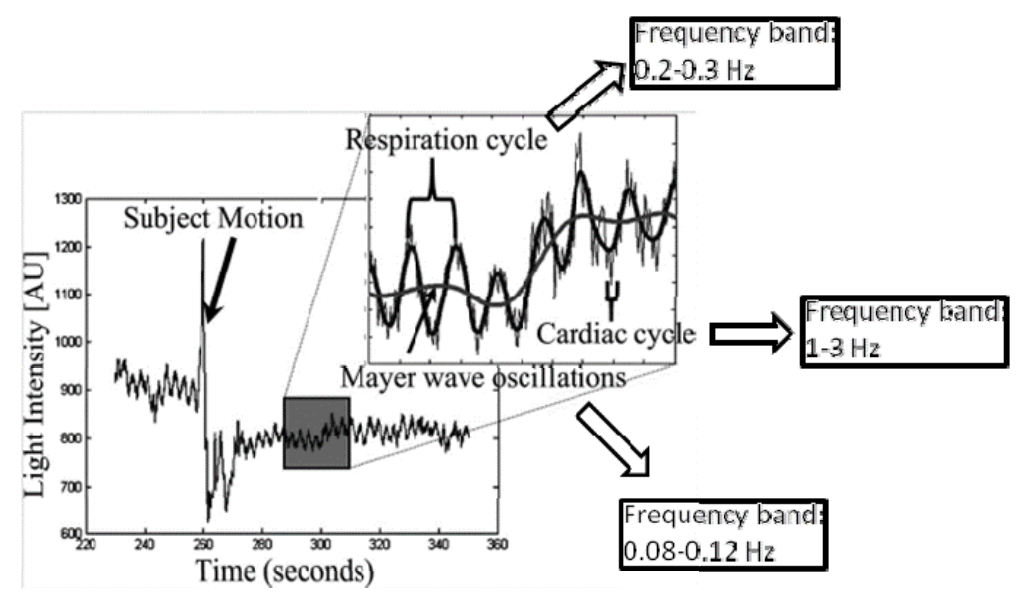

Figure 5: This figure shows the noise that is induced in the NIRS signal from respiratory cycles, cardiac cycles and Mayer wave oscillations. Also to note is the motion artifact that is present during this study due to subject motion. (Adapted from Huppert et. al (2009) ${ }^{[2]}$

\begin{tabular}{|l|l|l|}
\hline Physiological Signals & Response Timescale & Frequency Band \\
\hline Heart rate & 1 sec period & $1,2,3 \mathrm{~Hz}$ \\
\hline Respiration & & \\
& & $0.2-0.3 \mathrm{~Hz}$ \\
\hline
\end{tabular}




\begin{tabular}{|l|l|l|}
\hline Mayer waves & $\sim 10 \mathrm{sec}$ period & $0.08-0.12 \mathrm{~Hz}$ \\
\hline "Fast" signal & $\sim 0.050 \mathrm{sec}$ & $20+\mathrm{Hz}$ \\
\hline Hemodynamic response & $\sim 0.2-10 \mathrm{sec}$ & $0.1-5 \mathrm{~Hz}$ \\
& & \\
\hline
\end{tabular}

Table 2: Different types of biological signals and their frequency range.

Step 2: The filtered data is normalized further to provide the relative change by dividing the optical data (over the entire time series) by its mean across all five blocks.

$$
\text { Normalized DC data }=\frac{\phi(\mathrm{t}, \lambda)}{\phi_{o}(t)} \quad \text { Eqn.3 }
$$

Where $\phi(t, \lambda)$ is photon fluence at time $t$, and $\phi_{o}(t)$ is mean photon fluence at time $t$.

Step 3: The normalized data is then used to calculate change in optical density for each wavelength.

$\operatorname{delta}(\Delta)$ optical density $(\operatorname{doD})=-\log \frac{\phi(\mathrm{t}, \lambda)}{\phi_{o}(t)} \quad$ Eqn. 4

Step 4: The doD is used to calculate delta concentration from the Modified Beer-Lambert law as shown in Eq. 2, 3, 4 5, and 6.

The change in the amount of detected light is also known as the change in the optical density $(\Delta \mathrm{OD})$, which is linearly proportional to small changes in the absorption coefficient. Equation 4 shows how to calculate the change in optical density using the natural logarithm, where $\Phi_{0}$ denotes the average detected photon fluence of light through the tissue experiencing the 
absorption change. The details of the modified beer lambert law as well as the change in the optical density are provided in chapter 1 under the theory of NIRS.

Using this equation, the wavelength dependent absorption coefficients can then be calculated using the equations below.

$$
\begin{aligned}
& \Delta O D \lambda_{1}=\varepsilon_{H b R}^{\lambda 1} * L *[H b R]+\varepsilon_{H b O}^{\lambda 1} * L *[H b O] \quad \text { Eqn.5 } \\
& \Delta O D \lambda_{2}=\varepsilon_{H b R}^{\lambda 2} * L *[H b R]+\varepsilon_{H b O}^{\lambda 2} * L *[H b O] \quad \text { Eqn.6 }
\end{aligned}
$$

In these equations, $\varepsilon_{\mathrm{HbR}}^{\lambda 1} \& \varepsilon_{\mathrm{HbR}}^{\lambda 2}$ represent the extinction coefficients of deoxyhemoglobin at $830 \mathrm{~nm}$, which corresponds to wavelength $1\left(\lambda_{1}\right)$, and at $690 \mathrm{~nm}$ (wavelength $2, \lambda_{2}$ ). The extinction coefficients for oxy-hemoglobin are represented by, $\varepsilon_{\mathrm{HbO}}^{\lambda 1} \& \varepsilon \underset{\mathrm{HbO}}{\lambda 2}$, where $\lambda_{1}$ is $830 \mathrm{~nm}$ and $\lambda_{2}$ is $690 \mathrm{~nm}$. L represents the effective average pathlength of light through the tissue experiencing the absorption change. $\mathrm{L}$ can be broken down into the source detector separation, which is measured along the surface of the head, multiplied by a quantity known as the differential pathlength factor. ${ }^{[3]}$

$$
\Delta H b X=\left(e^{T} e\right)^{-1} e^{T}[\Delta O D] \quad \text { Eqn.7 }
$$

$\Delta \mathrm{HbX}$, in this equation represents a vector which contains the changes in oxy-hemoglobin ( $\mathrm{HbO})$ and the changes in deoxy-hemoglobin (HbR). The $e$ is a matrix which contains the absorption coefficients of both oxy-hemoglobin and deoxy-hemoglobin, at $690 \mathrm{~nm}$ and $830 \mathrm{~nm}$. $\Delta \mathrm{OD}$ is the vector of change in optical density calculated using equations 5 and 6 above, respectively.

$$
\Delta H b T=\Delta H b O+\Delta H b R \quad \text { Eqn. } 8
$$


The equation above shows the relative total hemoglobin concentration $(\Delta H b T)$, which is equal to the sum of the relative concentration changes in both oxy-hemoglobin $(\Delta \mathrm{HbO})$ and deoxy-hemoglobin $(\Delta \mathrm{HbR})$.

As mentioned below, the optical signals acquired in real time are processed using HomER $^{\mathrm{TM}}$ (Hemodynamic Evoke Response). HomER ${ }^{\mathrm{TM}}$ (is a Matlab ${ }^{\circledR}$ graphical user interface, which performs the filtering algorithms, normalization, and averaging of the signal acquired for each subject trial. HomER is a free public domain software offered, and can be found at (http://www.nmr.mgh.harvard.edu/PMI/resources/homer/home). HomER ${ }^{\text {TM }}$ also calculates both the change in the optical density, and the relative concentration changes in $\mathrm{HbO}, \mathrm{HbR}$, and $\mathrm{HbT}$. Once the individual trials have been filtered, normalized, and averaged, the averaged hemodynamic response can be extracted, according to its respective channel (source-detector pair), and saved for further post processing. Figure 6 below shows an image of the graphical user interface known as HomER ${ }^{\mathrm{TM}}$. Further details of HomER's functionality are given in Appendix B. The 30 second average was also compared to the 15 second average using HomER to assess if the thirty second block average is truly reflective of the hemodynamic response function throughout the thirty second average. The results of this comparison are given in Appendix C (Tables C1-C6). 


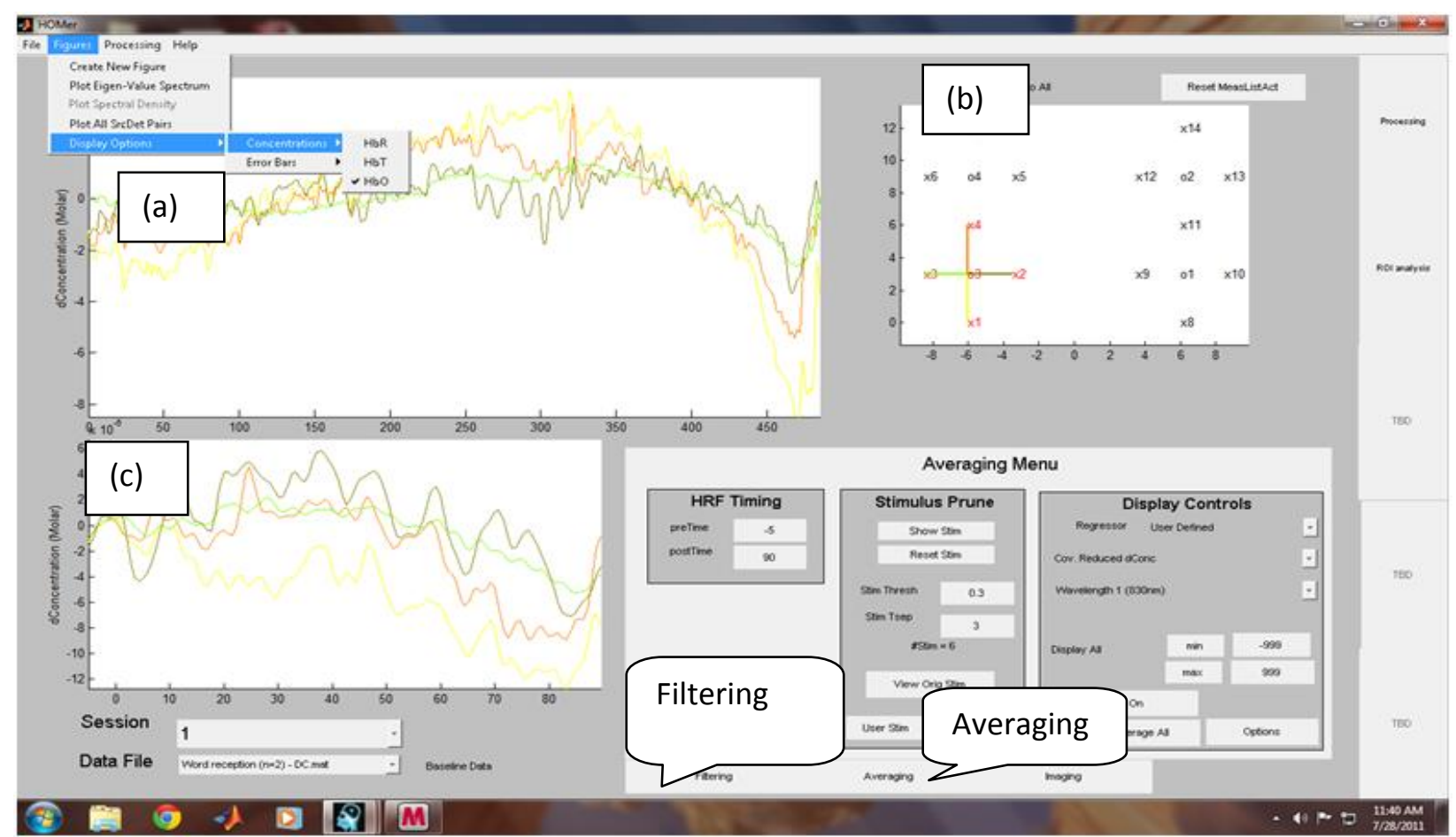

Figure 6. Screen shot of the HomER program. The layout of the HomER program is based around an interactive graphical display of the NIRS probe, shown in the upper right (b). The user specifies this probe geometry within the data file imported into HomER as described in the text. By selecting source (displayed as "x") or detector ("o") positions on this probe layout, the user navigates through the display of their data. The original data are shown in (a) and the average evoked response is shown in (c).

\subsubsection{Activation Analysis}

The acquired optical signals in real-time were processed using, HomER ${ }^{\mathrm{TM}}$ (Hemodynamic Evoked Response, http://www.nmr.mgh.harvard.edu/PMI/resources/homer/home) as well as inhouse developed codes in Matlab 2010a. HomER is unable to process more than one experimental trial at a time. Furthermore HomER cannot average more than one file together to give an average of more than one subject. In order to average the relative concentration changes of both $\mathrm{HbO}$ and $\mathrm{HbR}$ for all the subject trials $(\mathrm{n}=30)$, custom codes were developed in Matlab 
2010a. Data processed by HomER for all subjects were extracted, and averaged together using Matlab 2010a. Matlab codes were also developed to plot the averaged relative concentration changes for all subjects (Activation analysis). Additionally, Matlab codes were developed to perform both the functional connectivity and lateralization analysis on the averaged $(\mathrm{HbO}, \mathrm{HbR}$, and/or $\mathrm{HbT}$ ) data across all subject trials. Once the signals were filtered, the signals were processed and averaged across the five blocks, to give one 90 second block consisting of the relative change in hemoglobin concentration (dConc.) (i.e. across the three 30 seconds stimuli) for each subject and replicate. The averaged hemodynamic signals (in terms of $\mathrm{HbO}, \mathrm{HbR}$, or $\mathrm{HbT}$ ) from each subject and replicate was further averaged across all subjects and both replicates in order to obtain grant average signals for each paradigm. From 30 trials (i.e. 15 subjects $\times 2$ replicates), only 19 trials were used during the grand average in each paradigm, because 11 out of 30 trials had poor or weak optical signals. These poor/weak signals could be either from ambient light interference, hair interference, and/or poor scalp optode contact. Boxy® has a computer interface software that represents each source detector pair with a colored box. Once Imagent is turned on, the Boxy® software also begins to monitor the amount of light that is being received by the detectors. When a study takes place, the software was used to monitor the amount of light being received by each detector during the full length of the study. When the detectors are receiving light with a high signal to noise ratio, the interface shows green in the boxes representing individual source-detector pairs, yellow when the signal to noise level drops to a cautionary point and red when the signal is below the signal to noise ratio. For the 11 trials that were removed, the software showed red for the majority of the study, after it had commenced. 
Activation (or hemodynamic changes) was measured across each source detector channel (shown in Figure 1), for both the left and right fronto-temporal regions of the brain and plotted in terms of $\mathrm{HbT}$ (grand averaged across the 19 trials).

\subsubsection{Functional Connectivity Analysis}

Functional connectivity is fundamentally a statistical concept. Functional connectivity is defined as the "temporal correlation between spatially remote neurophysiological events". ${ }^{[35,36]}$ Functional connectivity measures simultaneous coupling between two time series. It does not give us information about whether two units are connected or not i.e. it is agnostic to the directional or causal relationship between two time series. Functional connectivity is often calculated between all elements of a system, regardless of whether these elements are connected by direct structural links. On the other hand effective connectivity attempts to describe or make inferences about the direction of influence between regions. ${ }^{[35,36]}$

$$
\text { Correlation coefficient }=\frac{\sum_{i=1}^{n}\left(X_{i}-\bar{X}\right)\left(Y_{i}-\bar{Y}\right)}{\sqrt{\sum_{i=1}^{n}\left(X_{i}-\bar{X}\right)^{2}\left(Y_{i}-\bar{Y}\right)^{2}}}
$$

The correlation analysis was performed by computing the Pearson's correlation coefficient, as shown in Eqn. 9 where $\bar{X}$ is the mean (across a 30-second stimuli) of the averaged hemodynamic response $(\mathrm{HbO}, \mathrm{HbR}$, or $\mathrm{HbT})$ corresponding to a given channel in the left temporal region; $\bar{Y}$ is mean (across a 30-second stimuli) of the averaged hemodynamic response ( $\mathrm{HbO}, \mathrm{HbR}$, or $\mathrm{HbT}$ ) corresponding to a given channel in the right temporal region; $X_{i}$ and $Y_{i}$ are the HRFs (HbO, $\mathrm{HbR}$, or $\mathrm{HbT}$ ) at each time sequence corresponding to the left and right hemispheres of the 
temporal region, respectively. Additionally, $n$ is the number of time sequence (limited by the NIRS instrument data acquisition speed) in each stimulus.

In the present study, functional brain connectivity was performed to characterize the ways in which the left and right, as well as posterior and anterior fronto-temporal regions were functionally integrated or works in synchrony in response to language-based cognitive stimuli. This study is carried out by computing zero lagged correlation to elucidate the correlation and synchrony in the hemodynamic response and hence the connectivity in the neuronal circuit between the left and right, and posterior and anterior fronto-temporal region.

Once the functional connectivity analysis is performed, a lateralization analysis is performed on the data from both paradigms to see which hemispheres are dominant in each channel during the three tasks in each paradigm.

\subsubsection{Lateralization Analysis}

Lateralization is the localization of function attributed to either the right side or left side of the brain. This means that the brain has a tendency for certain processes to be more highly developed on one side of the brain over the other (i.e. left or right). As humans, there is a tendency to use one half of the brain more than the other, which develops into "brain dominance" (the predisposition to think and act using either the left or right hemisphere predominantly) in early childhood. ${ }^{[53,54]}$ This dominance is determined by genetics, experiences, and environment. ${ }^{[53]}$ But, just because an individual has selected a dominant hemisphere does not mean that the other hemisphere is absent in thought processes and actions. Even though the brain does not trust its non-dominant half, the non-dominant hemisphere occasionally leads in thought 
processes and actions. ${ }^{[53]}$ The use of the laterality index allows for the determination of the dominant hemisphere to a given thought process or stimuli. The laterality index relies on total hemoglobin to determine dominance due to the fact that total hemoglobin takes into account all of the blood present in a given hemisphere at any time. The formula used to determine the laterality index $(L(t))$ is given below:

$$
L(t)=\frac{\mathrm{HbT}(\mathrm{t})_{\text {Left Temporal }}-\mathrm{HbT}(\mathrm{t})_{\text {Right Temporal }}}{\left|\mathrm{HbT}(\mathrm{t})_{\text {Left Temporal }}\right|+\left|\mathrm{HbT}(\mathrm{t})_{\text {Right Temporal }}\right|} \quad \text { Eqn. } 10
$$

The laterality index will be obtained from the grand averaged $\mathrm{HbT}$ data for 19 trials for each time point. These time-series laterality index values will further be averaged across each 30 -sec stimuli of each paradigm in order to obtain a single laterality index value for a given stimuli and paradigm. Based on the above formula, a positive laterality index corresponds to left temporal dominance, else a right temporal dominance. A near zero (i.e. $-0.25 \leq \mathrm{L}(\mathrm{t}) \leq 0.25$ ) laterality index value indicates there is no dominance (Bilateral dominance) between the channel pairs. ${ }^{[42,}$ 54]

\subsubsection{Regional Analysis}

A regional analysis was also performed on both the word expression paradigm, and the word reception paradigm. By performing a regional analysis, there are now four distinct regions that have been created. By creating four regions, each representing one language area of the brain, comparisons can be made between these regions, not only between hemispheres ( left vs. right) as in the channel-wise study, but also between language areas in the same hemisphere, and between language areas in other hemispheres ( i.e. left/right posterior/anterior regions). The main goal in performing the regional analysis was to see how the four language areas were 
connected during each paradigm, not just left vs. right hemispheres, but as mentioned previously, across all four regions. These comparisons were not possible when looking at the channel-wise data, since it is often difficult to try and compare individual channels in the same manor. Since each of the four regions described above were created from information from four channels (source-detector pairs), they are able to provide a general overview of how each region is functioning. Since a functional connectivity analysis was performed on the regional data, an activation analysis as well as a lateralization analysis was also performed to see if the information provided from the regional analysis was same or different with respect to the channel-wise data., The information provided from these analyses allows for a more general view of the data, since it is represented by only four regions, and not 16 channels. A full analysis of how the regional analysis is formulated is given in Appendix D. The four channel and three channel regional average and standard deviations for $\mathrm{HbO}, \mathrm{HbR}$, and $\mathrm{HbT}$ for both the word expression and word reception paradigm are given in tables D1-D6 of appendix D. Tables D7D12 are given in appendix D to show that channels 4 and 5 are to be considered significantly different based on comparison of these channels two channels to respective four channel mean. Tables D13-18 is given in appendix D to show that no channels are significantly different from respective regional average, after exclusion of channels 4 and 5. 


\section{5- Statistical Analysis:}

\subsection{1- Power Analysis}

As previously mentioned in section 3.4.1, 11 trials out of 30 were removed from the grand average for each paradigm due to weak optical signal. A power analysis was performed after the removals of these trials to determine how likely the grand average of 19 trials were to represent the entire population. Power analysis tells of the probability of rejecting the null hypothesis (null hypothesis: two stimuli are equal) when the null hypothesis is true. Power analysis depends upon three factors: difference between means, residual variance, and sample size. In order to determine the difference between means of two samples (i.e. stimuli 1 vs. stimuli 2), the Cohen's d value must be calculated. The Cohen's D value gives the measure of how different two means of two different stimuli are compared to the standard deviation within the stimuli. The Cohen's d value is calculated from the following formula:

$$
\text { Cohen's } d=\frac{\overline{x_{1}}-\overline{x_{2}}}{s}
$$

Where $\mathrm{s}$ is equal to:

$$
\sqrt{\frac{\left(n_{1}-1\right) s_{1}^{2}+\left(n_{2}-1\right) s_{2}^{2}}{n_{1}+n_{2}}}
$$

where $\mathrm{s}_{1}^{2}$ is equal to:

$$
\frac{1}{n_{1}-1} \sum_{i=1}^{n_{1}}\left(x_{1, i}-\bar{x}_{1}\right)^{2}
$$


and $\mathrm{s}_{2}{ }^{2}$ is equal to :

$$
\frac{1}{n_{2}-1} \sum_{i=1}^{n_{2}}\left(x_{2, i}-\bar{x}_{2}\right)^{2}
$$

In the following equations, $\bar{x}_{1}$ and $\bar{x}_{2}$ represent the mean of the hemodynamic response function across 30 seconds for each of the two stimuli. $x_{1, i}$ and $x_{2, i}$ represent the hemodynamic response functions at time $\mathrm{t}$ for each of the two stimuli. The term $n$ is the sample size for each of the two stimuli, which, for all cases in both paradigms n was equal to 19 . Using equations 11-14, the Cohen's d value was determined for $\mathrm{HbO}, \mathrm{HbR}$, and $\mathrm{HbT}$, for both the word expression and word reception paradigm. For the word expression paradigm, the Cohen's d values were 0.96 for $\mathrm{HbO}, 0.89$ for $\mathrm{HbR}$, and 0.93 for $\mathrm{HbT}$. For the word reception paradigm, the Cohen's d values were 0.91 for $\mathrm{HbO}, 0.94$ for $\mathrm{HbR}$, and 0.88 for $\mathrm{HbT}$. Using these values, along with the sample size of 19, the power of our experiments can be determined by using Table 3 below.

\section{Sample Size Required for a Two-Sample $t$ Test ${ }^{a}$ to Achieve a Given Level of Power for a Given Effect Size and Alpha of .05}

\begin{tabular}{|c|c|c|c|}
\hline \multirow[b]{2}{*}{ Power } & \multicolumn{3}{|c|}{ Effect Size (Cohen's $d$ ) } \\
\hline & .2 & .5 & .8 \\
\hline .25 & 84 & 14 & 6 \\
\hline .50 & 193 & 32 & 13 \\
\hline .60 & 246 & 40 & 16 \\
\hline .70 & 310 & 50 & 20 \\
\hline .80 & 393 & 64 & 26 \\
\hline .90 & 526 & 85 & 34 \\
\hline .95 & 651 & 105 & 42 \\
\hline .99 & 920 & 148 & 58 \\
\hline
\end{tabular}

Taken from Cohen (1977).

NOTE: Entry in the table is the sample size for each of the two groups.

Table 3: Power Analysis look-up table based on Cohen's predictive values for a $95 \%$ confidence interval. ${ }^{[55]}$ 
From the table, it can be seen that with a Cohen's d value greater than 0.8 , as is all the cases for both the word expression and word reception paradigm, with a sample size of 19 , that the power is 0.7 . In other words, power analyses indicated that the sample sizes of 19 in this study can provide $70 \%$ likelihood or higher for detecting high effect size $(>0.8)$.

\subsection{2- Consistency Test: Grand Average mean vs. Individual subject means}

In order to ensure that the grand average of 19 trials reflected the average of each individual subject, statistical analysis was performed. A paired student's t-test was used to confirm whether or not the grand average reflected each individual subject average. Using the ttest function in Microsoft Excel, with a 95\% confidence interval, and unequal variance, the pvalues were calculated for each individual subject's average compared to the total grand average of all the subjects' trials to check for consistency between individual subjects and the grand average. If the $\mathrm{p}$-values were less than $0.05\left(\mathrm{p}_{\mathrm{i}}<0.05\right)$ then the two mean sets were considered significantly different. The results from the consistency test showed there were no differences between any individual subject average and the grand average of 19 subject trials (i.e. $\mathrm{p}>0.05$ ).

\subsection{3- Differences in Hemodynamic Response Across Stimuli}

Once the grand averaged data was verified that it reflected the individual subject's data sets, the data was analyzed to see whether the three treatments were the same or different (i.e. the stimuli). The three stimuli for the Word Expression paradigm (Word Generation, Jaw Movement, and Rest) and the Word Reception paradigm (Forward Audio, Reverse Audio, Rest) were compared against one another within each paradigm to ascertain if the activation is significantly different with respect to stimuli. Hence, a three paired p-test is performed to 
determine statistically if there is any significant difference across the three pairs of data sets. The p-values computed for each paired stimuli were computed using both a student's t-test, which has been previously described. Another option was to employ the Analysis of Variance (ANOVA) test. However, ANOVA is typically applicable for large sample size ( $>25-30)$. Since our current sample size is 19, ANOVA analysis was not performed. The ANOVA was computed using Microsoft Excel software. The ANOVA analysis performed was a single factorial experimental design, with a 95\% confidence interval, with two tails. The analysis provides a test of hypothesis that each sample is drawn from the same underlying probability distribution against the alternative hypothesis that underlying probability distributions are not the same for all samples. A single factorial design was chosen for the ANOVA due to the fact that only two stimuli means are being compared at any given time. Once the p-values have been computed, either through a Student's t-test, or an ANOVA, the three paired p-test analysis was performed. The ANOVA p-values were not used due to the fact that the ANOVA is not suited for small sample sizes. Microsoft Excel indicates that if the sample size is smaller than 25, it is better to use the Student's T-test. This is due to the fact that the ANOVA has a greater probability of committing a Type I error ( rejecting null hypothesis when it is true). For the three paired $\mathrm{p}$ analysis, $\mathrm{p}$-values were computed using both ANOVA, and Student's T-test, but the three paired $\mathrm{p}$ analysis was performed using only those values computed from a Student's T-test The steps to compute the three paired p-values are given below:

1) The p-value (Stimuli 1 vs. Stimuli 2, Stimuli 1 vs. Stimuli 3, and Stimuli 2 vs. Stimuli 3) of each 30 second stimuli for $(\mathrm{HbO}, \mathrm{HbR}$, and $\mathrm{HbT})$ is computed.

2) The p-value of the given pair of data is sorted in ascending order. 
3) Each p-value is compared with the inequality given by Eqn. 15 If the inequality given by Eqn. 15 is not met then the correlation is considered not significant at the given statistical level of significance.

$$
p_{i} \leq \frac{\alpha}{1+k-i}
$$

Where $p_{i}$ corresponds to the $\mathrm{p}$-value of the particular pair among the total numbers of pair; $\alpha$ is the statistical level of significance; $\mathrm{k}$ is the total number of pair in the comparison and $i$ corresponds to the particular pair among the total numbers of pair.

Summary

A NIRS based optical imaging system (Imagent ISS Inc., Champaign IL), provided by Miami Children's Hospital was used to conduct both language studies. 15 normal right handed adult volunteers were recruited to participate in both language studies. Using facilities provided by Miami Children's Hospital, and custom built head caps (at the Optical Imaging Laboratory), both experiments were carried out on the adult population recruited for the study. Data analysis was carried out using a software known as HomER, a free public domain software offered, which can be found at (http://www.nmr.mgh.harvard.edu/PMI/resources/homer/home). Once data analysis was complete, custom built Matlab 2010a codes were used to perform statistical analyses on the grand averaged files of 15 subjects with 2 replicates for both language studies. Of the 30 trials, 11 trials were removed due to poor signal to noise ratio (as determined by a graphical user interface software $\mathrm{BOXY}^{\circledR}$ ). Custom codes were also implemented to carry out the activation analysis, functional connectivity analysis, and lateralization analysis for both language studies. The results of these analyses are presented in the following chapters. 
Chapter 4- Results and Discussion: Word Expression Paradigm

The following chapter describes in detail the results of the word expression paradigm. The activation, functional connectivity, and lateralization for the channel-wise study are first described. Then the regional analysis results from the word expression paradigm in terms of activation, functional connectivity, and lateralization are described. The chapter then focuses on the discussion of the results of both the channel-wise as well as regional analysis. The results from this chapter are based on a word expression paradigm, which is focused on the generation of speech in the fronto-temporal regions of the brain.

\subsection{Word Expression Paradigm}

\subsection{1-Activation Analysis}

Figure 7 provides the activation plots (in terms of both $\mathrm{HbO}$ (red) and HbR (blue) for all the channels in the left and right temporal regions obtained in response to the word expression paradigm. Observing channels (5-8) (both left and right hemisphere) which correspond to the anterior fronto-temporal region (encompassing Broca's area), the oxygenated hemoglobin activation varies across all three stimuli, with significant differences in activation across all three stimuli (i.e. $\mathrm{p}<0.05$ from 3-paired t-test). Tables E1-E3 in Appendix E) provides p-values for $\mathrm{HbO}, \mathrm{HbR}$, and $\mathrm{HbT}$, respectively, across all channels for all three stimuli during the word expression paradigm in order to demonstrate these significant differences. 


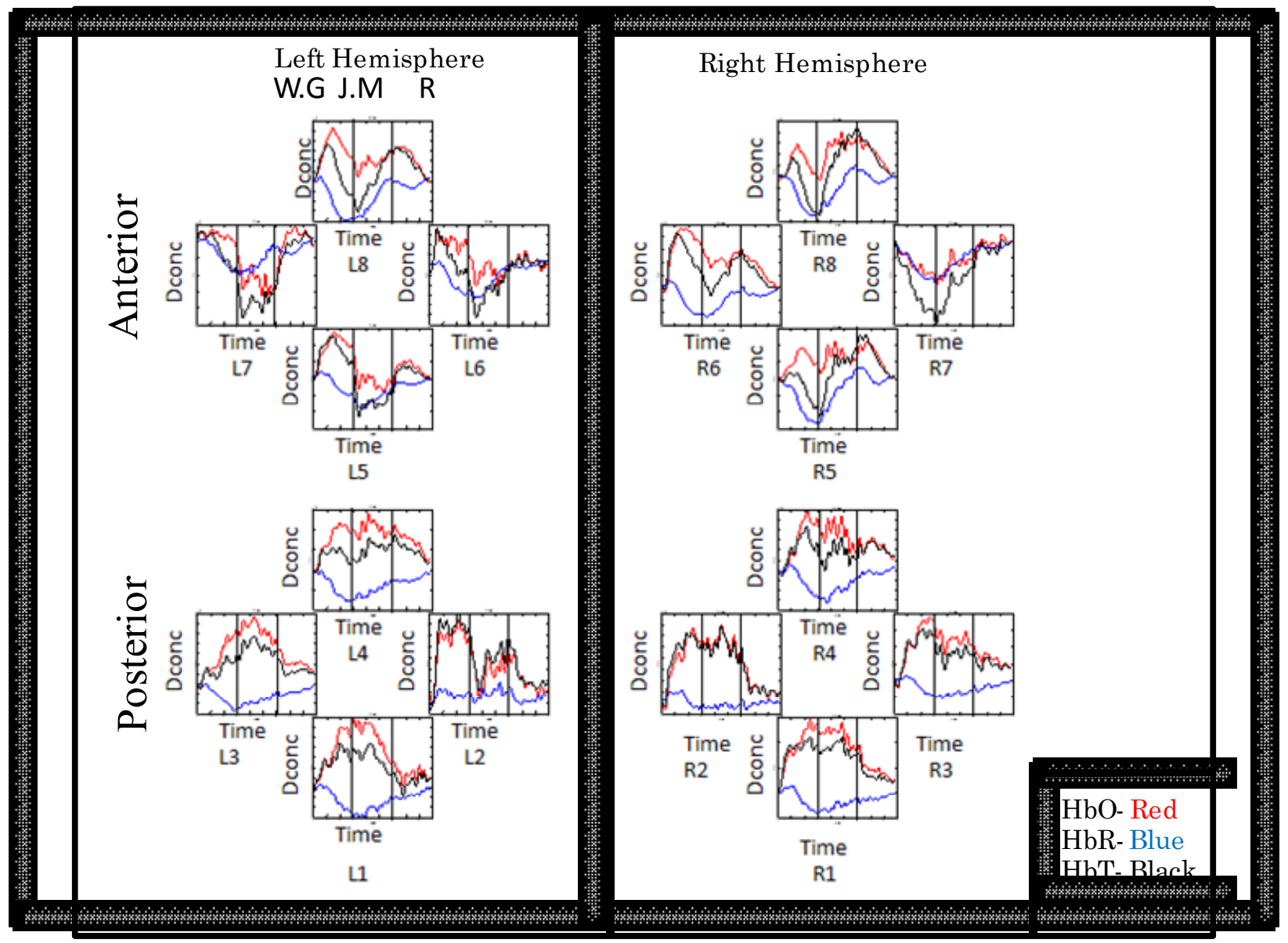

Figure 7. Word Expression Paradigm activation plots. The plots show oxygenated (red) and deoxygenated (blue) hemoglobin activation in the left and right fronto-temporal regions of the brain during the Word Expression Paradigm. All plots are shown on the same scale. The y-axis corresponds to the relative change in hemoglobin, while time is shown on the x-axis. The plots are divided into the three 30 second stimuli (from left to right), Word Generation (W.G), Jaw Movement (J.M), Rest (R). All plots are set to the same scale, with the y scale ranging from $\left(-2 \times 10^{-5}-2 \times 10^{-5}\right)$

Observing channels (1-4) which correspond to the posterior fronto-temporal region (left and right hemisphere) (encompassing Wernicke's area), the activation for oxygenated hemoglobin (HbO, red) again differs across all three stimuli. In both regions, the activation tends to be highest during the word generation stimuli in the majority of the channels, while the 
activation tends to be lower during the rest stimuli, where it tends to return to the baseline. The extent of activation is based on comparing activation task means to the baseline rest means for all channels. Table 4 provides the mean concentrations of $\mathrm{HbO}, \mathrm{HbR}$ and $\mathrm{HbT}$ for each 30 second stimuli, across all channels during the word expression paradigm. Looking at Table 4 the highest activation occurs during the word generation task, for $\mathrm{HbO}$, in two channels in the left hemisphere of the anterior fronto-temporal region (encompassing Broca's area) (channel L8), and in channel R6 in the right homologue of Broca's region during the same stimuli.

\begin{tabular}{|c|c|c|c|c|c|c|c|c|c|}
\hline \multicolumn{10}{|c|}{ Word Expression Paradigm Mean Values for each 30 second stimuli } \\
\hline \multirow[t]{2}{*}{ Channel } & \multicolumn{3}{|c|}{ Word Generation } & \multicolumn{3}{|c|}{ Jaw Movement } & \multicolumn{3}{|c|}{ Rest } \\
\hline & $\mathrm{HbO}$ & $\mathrm{HbR}$ & $\mathrm{HbT}$ & $\mathrm{HbO}$ & $\mathrm{HbR}$ & $\mathrm{HbT}$ & $\mathrm{HbO}$ & $\mathrm{HbR}$ & $\mathrm{HbT}$ \\
\hline $\mathrm{L} 1$ & 7.1E-06 & $-1.8 \mathrm{E}-06$ & $5.2 \mathrm{E}-06$ & $1.1 \mathrm{E}-05$ & $-4.2 \mathrm{E}-06$ & $6.0 \mathrm{E}-06$ & $2.8 \mathrm{E}-06$ & $-1.4 \mathrm{E}-06$ & $1.3 \mathrm{E}-06$ \\
\hline $\mathrm{L} 2$ & $1.0 \mathrm{E}-05$ & $1.3 \mathrm{E}-06$ & $1.2 \mathrm{E}-05$ & $6.0 \mathrm{E}-06$ & $1.6 \mathrm{E}-06$ & 7.7E-06 & $4.2 \mathrm{E}-06$ & $7.3 \mathrm{E}-07$ & $4.9 \mathrm{E}-06$ \\
\hline L3 & $4.8 \mathrm{E}-06$ & $-2.2 \mathrm{E}-06$ & $2.6 \mathrm{E}-06$ & $1.1 \mathrm{E}-05$ & $-3.5 \mathrm{E}-06$ & $7.3 \mathrm{E}-06$ & $4.3 \mathrm{E}-06$ & $-1.5 \mathrm{E}-06$ & $2.9 \mathrm{E}-06$ \\
\hline L4 & 7.5E-06 & $-4.1 \mathrm{E}-06$ & $3.4 \mathrm{E}-06$ & $1.0 \mathrm{E}-05$ & $-5.1 \mathrm{E}-06$ & $5.2 \mathrm{E}-06$ & $6.9 \mathrm{E}-06$ & $-2.0 \mathrm{E}-06$ & $4.9 \mathrm{E}-06$ \\
\hline $\mathrm{L} 5$ & $1.9 \mathrm{E}-05$ & $-2.5 \mathrm{E}-06$ & $1.6 \mathrm{E}-05$ & $-1.5 \mathrm{E}-06$ & $-1.3 \mathrm{E}-05$ & $-1.4 \mathrm{E}-05$ & $6.0 \mathrm{E}-06$ & $-2.6 \mathrm{E}-06$ & $3.4 \mathrm{E}-06$ \\
\hline L6 & $1.0 \mathrm{E}-05$ & $-6.3 \mathrm{E}-06$ & $3.7 \mathrm{E}-06$ & $-3.5 E-06$ & $-9.7 E-06$ & $-1.3 \mathrm{E}-05$ & $1.2 \mathrm{E}-06$ & $-1.4 \mathrm{E}-07$ & $1.1 \mathrm{E}-06$ \\
\hline $\mathrm{L} 7$ & $1.6 \mathrm{E}-06$ & $-3.5 \mathrm{E}-06$ & $-1.9 \mathrm{E}-06$ & $-1.1 \mathrm{E}-05$ & $-6.4 \mathrm{E}-06$ & $-1.8 \mathrm{E}-05$ & $5.5 \mathrm{E}-07$ & $-2.0 \mathrm{E}-06$ & $-1.4 \mathrm{E}-06$ \\
\hline L8 & $1.6 \mathrm{E}-05$ & $-9.6 \mathrm{E}-06$ & $6.3 \mathrm{E}-06$ & 9.3E-06 & $-9.2 \mathrm{E}-06$ & $8.5 \mathrm{E}-08$ & $1.1 \mathrm{E}-05$ & $-1.2 \mathrm{E}-06$ & $9.4 \mathrm{E}-06$ \\
\hline R1 & $9.2 \mathrm{E}-06$ & $\begin{array}{l}-9.7 E-07 \\
\end{array}$ & $8.2 \mathrm{E}-06$ & $1.1 \mathrm{E}-05$ & $-2.7 \mathrm{E}-06$ & $8.7 E-06$ & $4.6 \mathrm{E}-06$ & $-1.1 \mathrm{E}-06$ & $3.5 \mathrm{E}-06$ \\
\hline $\mathrm{R} 2$ & $8.0 \mathrm{E}-06$ & $7.4 \mathrm{E}-07$ & $8.8 \mathrm{E}-06$ & 1.1E-05 & $-6.8 \mathrm{E}-07$ & 1.0E-05 & $3.1 \mathrm{E}-06$ & $-1.5 \mathrm{E}-07$ & $3.0 \mathrm{E}-06$ \\
\hline R3 & $4.3 \mathrm{E}-06$ & $-4.3 \mathrm{E}-07$ & $3.9 \mathrm{E}-06$ & $4.9 \mathrm{E}-06$ & $-1.6 \mathrm{E}-06$ & $3.2 \mathrm{E}-06$ & $2.5 \mathrm{E}-06$ & $-5.6 \mathrm{E}-07$ & $2.0 \mathrm{E}-06$ \\
\hline R4 & $5.7 \mathrm{E}-06$ & $-1.5 \mathrm{E}-06$ & $4.2 \mathrm{E}-06$ & $7.2 \mathrm{E}-06$ & $-4.1 \mathrm{E}-06$ & $3.1 \mathrm{E}-06$ & $4.6 \mathrm{E}-06$ & $-3.9 \mathrm{E}-07$ & $4.2 \mathrm{E}-06$ \\
\hline R5 & $5.0 \mathrm{E}-06$ & $-6.8 \mathrm{E}-06$ & $-1.8 \mathrm{E}-06$ & $6.4 \mathrm{E}-06$ & $-4.3 \mathrm{E}-06$ & $2.1 \mathrm{E}-06$ & $6.0 \mathrm{E}-06$ & $5.3 \mathrm{E}-07$ & $6.6 \mathrm{E}-06$ \\
\hline R6 & 1.7E-05 & $-4.1 \mathrm{E}-06$ & $1.3 \mathrm{E}-05$ & $1.2 \mathrm{E}-05$ & $-6.5 \mathrm{E}-06$ & $5.0 \mathrm{E}-06$ & $7.1 \mathrm{E}-06$ & $-2.1 \mathrm{E}-06$ & $5.0 \mathrm{E}-06$ \\
\hline R7 & $-6.4 \mathrm{E}-06$ & $-6.8 \mathrm{E}-06$ & $-1.3 \mathrm{E}-05$ & $-7.4 \mathrm{E}-06$ & $-7.0 \mathrm{E}-06$ & $-1.4 \mathrm{E}-05$ & $-5.8 \mathrm{E}-07$ & $-1.5 \mathrm{E}-06$ & $-2.1 \mathrm{E}-06$ \\
\hline R8 & $5.9 \mathrm{E}-06$ & $-8.5 E-06$ & $-2.6 \mathrm{E}-06$ & $1.3 \mathrm{E}-05$ & $-5.2 \mathrm{E}-06$ & 7.6E-06 & $9.2 \mathrm{E}-06$ & $-9.8 \mathrm{E}-08$ & $9.1 \mathrm{E}-06$ \\
\hline
\end{tabular}

Table 4: A table of the mean values for each of the three stimuli across all 16 channels for HbO, HbR, and HbT during the Word Expression Paradigm. The red font indicates the channel which recorded the largest amplitude of change for both the left and right hemisphere.

Observing channels (5-8), for deoxygenated hemoglobin (HbR, blue) the activation also differs across all three stimuli, with all three stimuli showing significant differences $(p<0.05)$. The activation tends to drop during the first stimuli, then rises through the jaw movement stimuli, and 
finally returns to baseline during the rest stimuli. Observing channels (1-4), there are significant differences in activation across the three stimuli for deoxygenated hemoglobin ( $\mathrm{HbR}$, blue) (TableE2 in Appendix E). Observing table 4 which shows the mean across each stimuli for each channel for $\mathrm{HbO}, \mathrm{HbR}$, and $\mathrm{HbT}$, the highest activation across all three tasks occurred in the anterior regions (both left and right, $\mathrm{Ch} .5-8$ ) for $\mathrm{HbO}$, as well as $\mathrm{HbT}$. Figure 7 also provides the activation plots (in terms of $\mathrm{HbT}$ ) for each channel in the left and right temporal regions obtained in response to the word expression paradigm. The $\mathrm{HbO}$ plot followed a similar trend as the $\mathrm{HbT}$ in all the experiments, and hence these activation plots also represent the same trends as in $\mathrm{HbO}$ data. Observing the channels (5-8) corresponding to the anterior fronto-temporal region (which encompass Broca's area, and its right hemisphere analog) the activation varies across all the three stimuli (i.e. Word generation vs. Jaw movement, Word generation vs. Rest, and Jaw movement $v s$. Rest) (Tables E1-E3 of Appendix E. Observing the channels (1-4) corresponding to the posterior fronto-temporal region (encompassing Wernicke's area, and its right hemisphere analog), there are significant difference between the word generation and jaw movement's activation plot $(\mathrm{p}<0.05)$.

The activation results from the word expression paradigm are consistent with previous findings from other groups. (Cannestra et.al ${ }^{[37]}$, Schecklman et. al ${ }^{[41]}$, Ehlis et. al ${ }^{[38,51]}$, Petersson et. al ${ }^{[57]}$, and Frith et. al ${ }^{[58]}$ ) all showed significant differences between each of their stimuli in regards to language in the anterior fronto-temporal region (encompassing Broca's area). Cannestra et. al (2000) reported a trend in activation plots, calling it a "boxcar trend", in response to a word naming task in Broca's region. ${ }^{[56]}$ This trend can also be seen in the activation plots presented in figure 8 for channels (5-8), which correspond to Broca's region, as 
well as in channels (1-4), which correspond to Wernicke's region. The "Boxcar" trend is simply a rising and falling of the plot line, where it creates the effect of a hump or a boxcar. Furthermore, Cannestra et. al(2000) also reported in the same paper that Broca's region tends to show higher activation across all stimuli. ${ }^{[56]}$ For the word expression study, the highest activations (mean average) found were occurring in channels which correspond to Broca's region (Ch5-Ch8). In another study performed by Schecklmann et. al (2008), who performed a verbal fluency task in part of the temporal cortex using NIRS, reported seeing increases in both $\mathrm{HbO}$ and $\mathrm{HbT}$ for the word generation task, and a marked decrease in HbR for the same task. ${ }^{[41]}$ Furthermore their results showed that there were differences in activation across the three stimuli they used, and also found the highest activation occurred during the word generation task, which is in line with the findings of Cannestra et. al (2000), who used intra-operative intrinsic signals. Ehlis et. al (2007) found that during a verbal fluency task, when compared to rest tasks, the activation in terms of $\mathrm{HbO}$ was much higher, although they did not specifically target the anterior fronto-temporal region (encompassing Broca's area), but rather the fronto-temporal region, which also encompasses parts of Broca's region. ${ }^{[38]}$ Furthermore they reported that the word generation task (word naming task) showed higher activation, than their control task, which was to recite categories of words written in front of them. The same research group also reported differences across all three stimuli in this region with regards to the means of activation, comparatively.

These findings seem to be in line with the results from our activation analysis during the word expression paradigm, where there were differences in all three stimuli (word generation v. jaw movement, word generation $\mathrm{v}$. rest, and jaw movement v. rest) across all 
channels (see Tables E1-E3 of Appendix E. Furthermore, these findings are also consistent with the fact that during the word expression paradigm, the highest activation seen in any channel (based on the mean of the stimuli for each channel, for $\mathrm{HbO}, \mathrm{HbR}, \mathrm{HbT}$ ), when comparing the activation tasks (word generation, jaw movement) to the control rest task, was found during the word generation task, for HbO, in Broca's region (as shown in Table 4).

Petersson et. al (2004) presented grammatical and non-grammatical strings to subjects, focusing on the anterior fronto-temporal region (encompassing Broca's area), and found that Broca's area became activated during a grammatical task. Furthermore the activation was marked by increases in $\mathrm{HbO}$, and decreases in HbR. ${ }^{[57]}$ Frith et. al (1991) who performed word generation tasks on the prefrontal and temporal cortices, concluded that word generation was associated with bilateral activation of the fronto-temporal cortex ${ }^{[58]}$ All of these findings are in accordance with the results from the current word expression study, where there is marked increase in both $\mathrm{HbO}$ and $\mathrm{HbT}$ during a word generation task, with a decrease in $\mathrm{HbR}$ during the same task. Also the highest activations during the word expression paradigm occur during a word generation task, and are found to originate in Broca's region. In summary, all the stimuli presented tend to differ with respect to one another, not just in Broca's region but across all channels. Cannestra et al (2000) reported that during a word generation task, Wernicke's region tends to become activated as well, when comparing their activation tasks to their control task. They concluded that Wernicke's region becomes activated, but not to the extent that Broca's region activates, when comparing activation tasks to control tasks. These findings lead Cannestra et. al(2000) to believe that Wernicke's region is more dynamic than previously thought. ${ }^{[56]}$ Observing Figure 7 for the word expression paradigm, channels 1-4, which 
correspond to Wernicke's region, show some activation. This activation is less than those for channels 5-8 though, which correspond to Broca's region, when comparing activation tasks (word generation, jaw movement) to the control resting task.

The results from the word expression study show that during the verbal fluency task (word generation task) there is an increase in the relative concentrations of $\mathrm{HbO}$ across all channels, and all four regions. During the verbal fluency task (word generation task) there is also a decrease in the relative concentration of $\mathrm{HbR}$ across all channels, and all four regions. This increase in $\mathrm{HbO}$, and decrease in HbR signifies that there is possibly a high demand for oxygen by the brain during the generation of words.

\subsection{2 -Functional Connectivity Analysis}

Figure 8 shows the ( left vs. right) functional connectivity results of $\mathrm{HbO}$ for the three stimuli in the temporal regions of the brain during the word expression paradigm. Observing channels 5-8, which correspond to the anterior fronto-temporal region (encompassing Broca's area), there is no change in the connectivity pattern (i.e. extent of correlation between left and right hemispheres as illustrated by connectivity diagram) during the jaw movement stimuli vs. the rest stimuli. Only in channels $1-4$, which correspond to the posterior fronto-temporal region (encompassing Wernicke's area) are there observed differences in connectivity patterns when comparing the jaw movement stimuli against the rest stimuli. Observing channels 6,7 , and 8 during the word generation stimuli there is a difference in the connectivity patterns when comparing this stimuli to both the jaw movement and rest stimuli. These channels are thought to 
contribute to the language task independent of the jaw movement. Observing channels 1-4 which correspond to the posterior fronto-temporal region (encompassing Wernicke's area), the connectivity patterns differ across all stimuli.

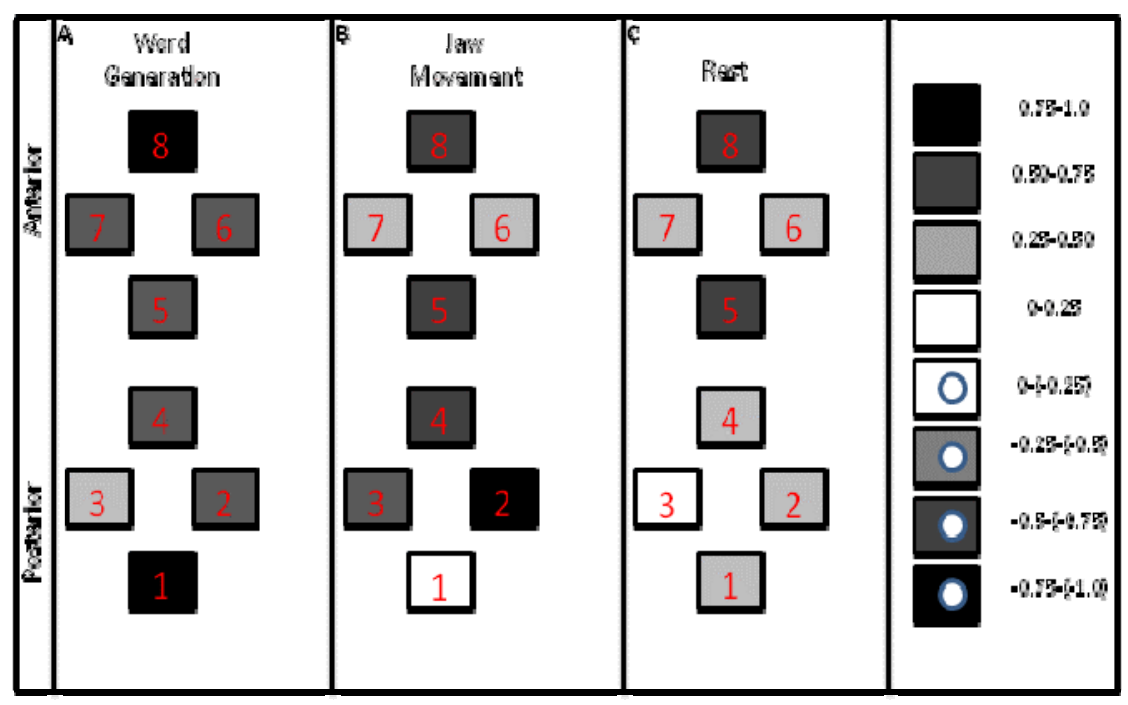

Figure 8. Oxy-hemoglobin Functional Connectivity Diagram. The diagram shows the correlation for channels 1-8 between the left and right hemisphere during the Word Expression Paradigm for HbO. The boxes are shaded according to the scale on the right hand of the figure, which corresponds to the correlation values calculated for the left and right hemisphere during the Word Expression Paradigm.

Figure 9 shows the ( left $v s$. right) functional connectivity results of $\mathrm{HbR}$ for the three stimuli in the temporal regions of the brain during the word expression paradigm. Observing channels 6 and 7, which correspond to the anterior fronto-temporal regions (encompassing Broca's area) there are differences in these channels when comparing the connectivity patterns of the jaw movement stimuli to rest stimuli. Furthermore, observing channels 1-4 which correspond to the posterior fronto-temporal region (encompassing Wernicke's area), there are differences between the connectivity patterns when comparing the jaw movement stimuli to the rest stimuli. Observing channels 5, 7, and 8 during the word generation stimuli, there are differences in the 
connectivity patterns of these channels when comparing to both the jaw movement stimuli, and the rest stimuli. Only channel 4 shows a difference in the connectivity pattern during word generation stimuli when comparing it to both the jaw movement and rest stimuli.

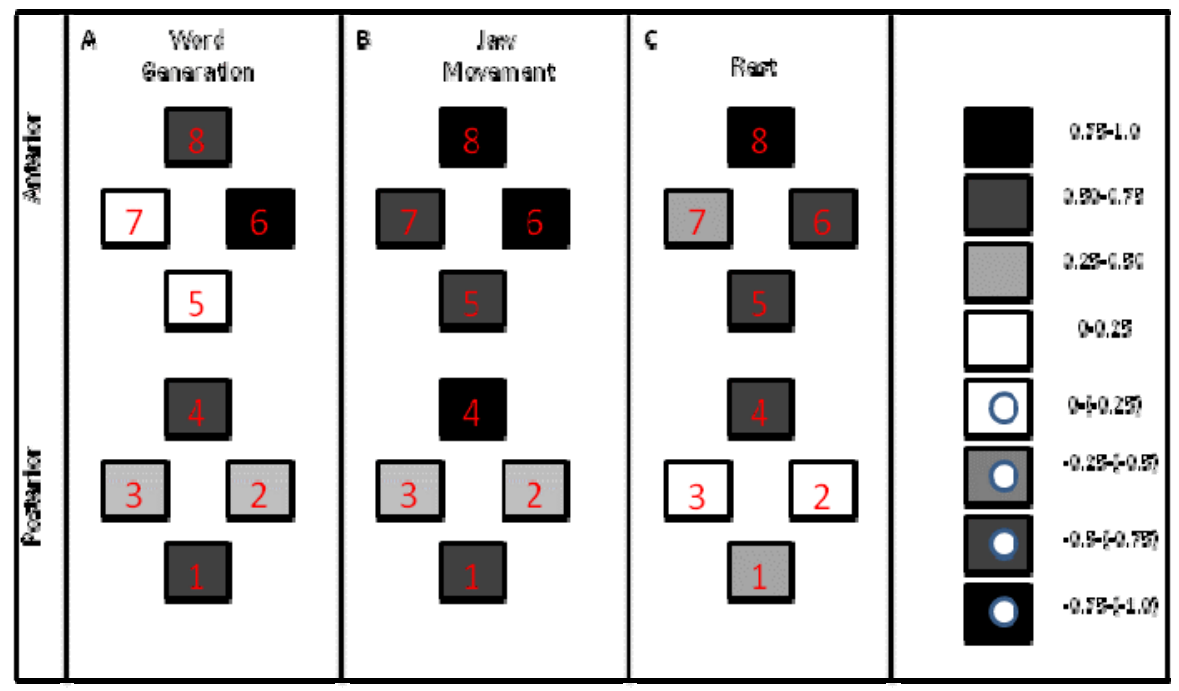

Figure 9. Deoxy-hemoglobin Functional Connectivity Diagram. The diagram shows the correlation for channels 1-8 between the left and right hemisphere during the Word Expression Paradigm for HbR. The boxes are shaded according to the scale on the right hand of the figure, which corresponds to the correlation values calculated for the left and right hemisphere during the Word Expression Paradigm.

Figure 10 shows the (left vs. right) functional connectivity results of $\mathrm{HbT}$ for the three stimuli in the temporal regions of the brain during the word expression paradigm. Observing channels 5 and 6 there are differences in the connectivity patterns of these two channels when comparing the word generation stimuli to the jaw movement stimuli. Observing channels 6 and 7, and channels 2 and 3, there are again differences in the connectivity pattern of these channels during jaw movement stimuli comparing to rest stimuli. Observing the connectivity patterns of word generation vs. jaw movement in the posterior fronto-temporal region, channel 2 is the only channel in this region that shows a difference. 


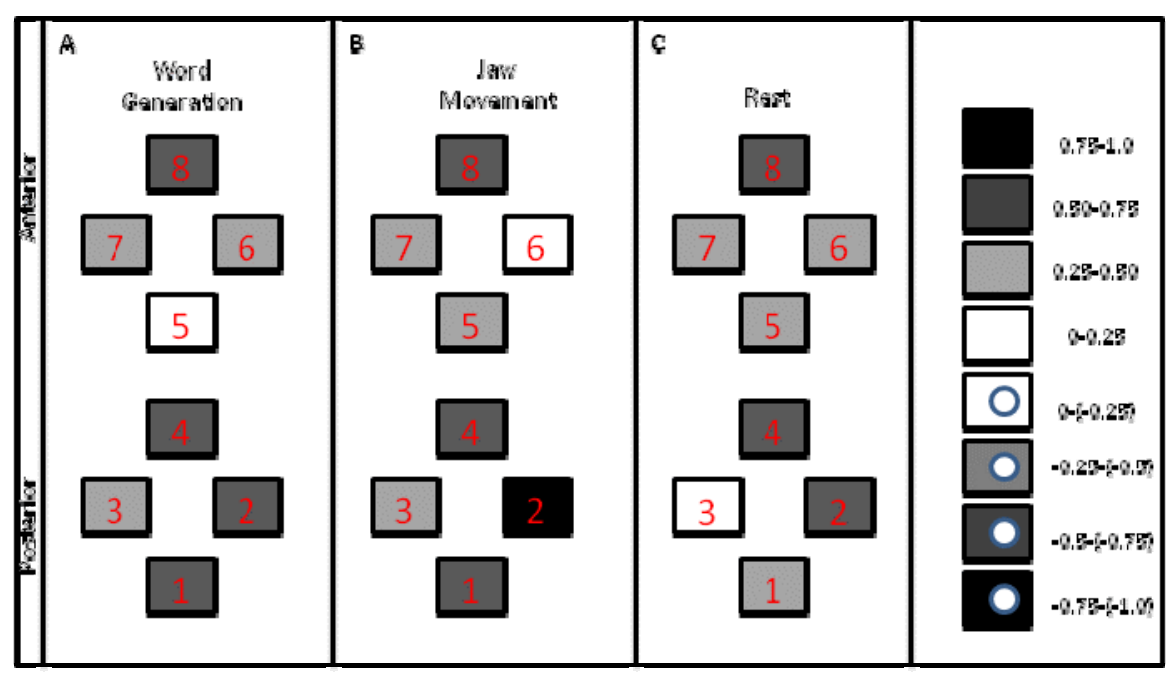

Figure 10.Total Hemoglobin Functional Connectivity Diagram. The diagram shows the correlation for channels 1-8 between the left and right hemisphere during the Word Expression Paradigm for HbT. The boxes are shaded according to the scale on the right hand of the figure, which corresponds to the correlation values calculated for the left and right hemisphere during the Word Expression Paradigm.

Overall the both the anterior and posterior fronto-temporal regions (encompassing both Broca's and Wernicke's area) show a stronger correlation during the word generation task, compared to the rest task for HbO. Zhang et. al (2010) using NIRS described similar results when comparing a language related verbal fluency task to a resting state. ${ }^{[35]}$ In their study, they found that while there is significant correlation during a rest task, the correlation between language areas becomes significantly higher during a language task. ${ }^{[35]}$

In summary only in the anterior fronto-temporal region, the region encompassing Broca's area, are there changes in the connectivity patterns (i.e. extent of correlation between left and right hemispheres as illustrated by connectivity diagrams), which occur when comparing the word generation stimuli to both the jaw movement and rest task. These changes in connectivity patterns were not observed in the posterior fronto-temporal region, the region which encompasses Wernicke's area. This change in connectivity patterns signify that the brain is 
using a different neuronal pathway to process language during the word generation task, than when performing a jaw movement task, or baseline rest. During the word generation task, the two hemispheres become strongly correlated, which would suggest that these two regions (left and right) seem to work with one another to some extent in the formulating and generating words. While during the jaw movement task, the two hemispheres of the anterior region become less correlated for $\mathrm{HbO}$, but there is a strong correlation for $\mathrm{HbR}$, which would suggest again a new pathway being utilized in these two regions. Interestingly though, when observing figure 8 in the anterior region for $\mathrm{HbO}$, there is no change in the amount of correlation or the connectivity pattern observed for either the jaw movement task, or the rest task, which implies that the pathways taken during these two tasks are relatively the same.

\subsection{3 -Lateralization Analysis}

Figure 11 depicts the results from the cortical lateralization study in the fronto-temporal region of the brain during the word expression paradigm. The anterior fronto-temporal region encompassing the Broca's area in the left hemisphere shows more dominance during the word generation (or activation) stimuli. This result concurs with the differences in the amplitude activation (mean, refer to table 3) between the left and right hemispheres during the word generation stimuli. There was no dominance (or bilateral activation) in the posterior frontotemporal region encompassing Wernicke's area during word generation stimuli. 


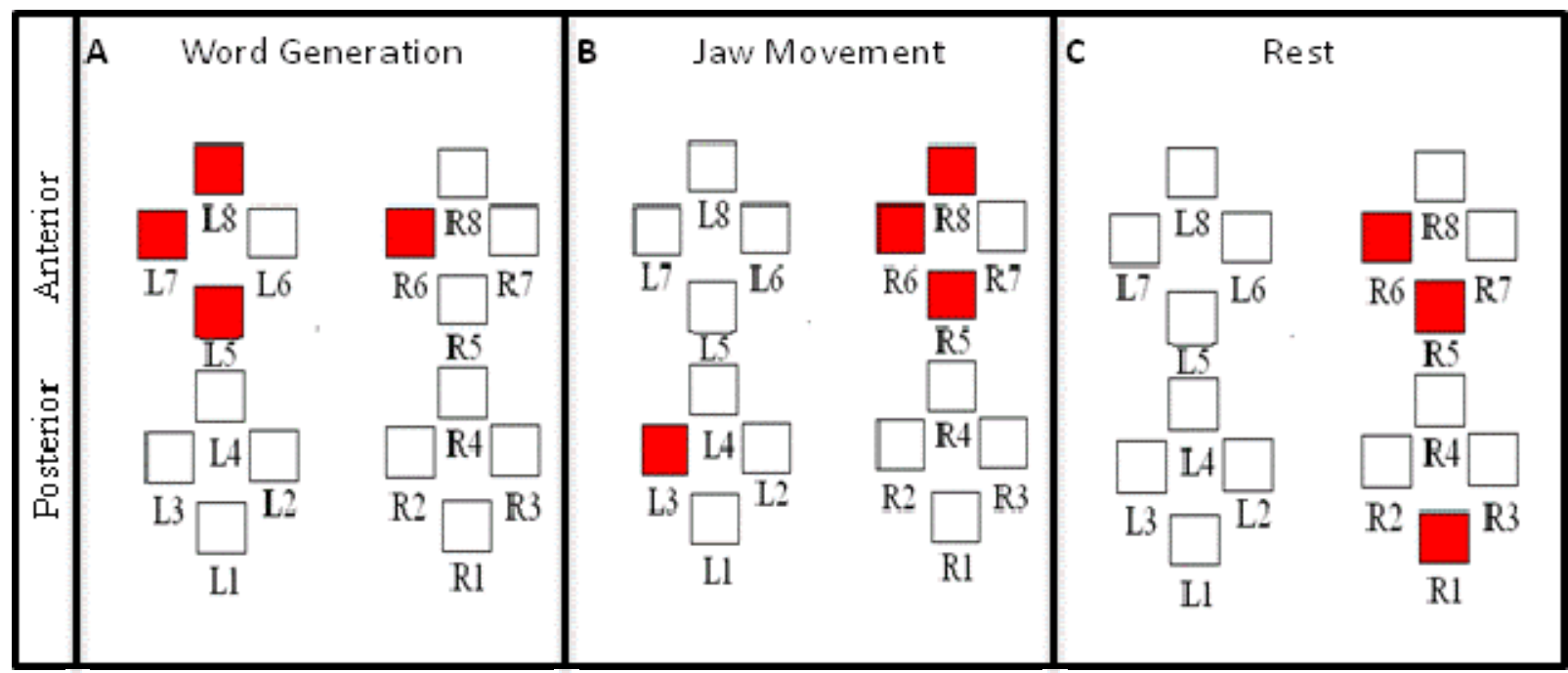

Figure 11. Lateralization Index Diagram. The following diagram shows the lateralization results for the three stimuli during the Word Expression Paradigm in the temporal region. The boxes that are shaded correspond to a dominate channel. Those boxes left unshaded correspond to a bilateral lateralization index( no dominance).

This is supported by the amplitude of activation (mean, table 3) in posterior frontotemporal region (encompassing Wernicke's area and its right hemisphere analog) in the left and right hemispheres during the word generation stimuli, which were relatively close together. This is further supported by the correlation observed in this region during word generation stimuli, where a moderately positive correlation was observed (figure 10).

During the jaw movement, the right anterior fronto-temporal region (encompassing right analog of Broca's region) was dominant. This also concurs with the differences in the amplitude of activation (mean, refer to table 3 ) during this task in both left and right hemispheres, where the mean was higher amongst the channels corresponding to the right anterior fronto-temporal region (encompassing right analog of Broca's area), rather than the left anterior region encompassing Broca's area. During this task it so happens that there is also strong positive correlations between 
the posterior fronto-temporal regions encompassing Wernicke's area and its right hemisphere analog (during jaw movement, as shown in figure 10). During the rest conditions, the right temporal region was relatively dominant over the left temporal region. This is an outcome of the differences in the (left vs. right) temporal connectivity across multiple channels in Broca and Wernicke region, where there was only a minimal positive correlation as observed from connectivity studies, observed in those channels showing dominance (Ch.1,Ch.5,Ch.6), as well as an outcome of the differences in the (left vs. right) temporal amplitude of activation (mean, refer to table 3) during the rest task in these channels.

During the language task (word generation) in the word expression paradigm, the left hemisphere shows more lateralization (dominance) than the right hemisphere. Similar results were also reported by Zhang et. al(2010) who used a verbal fluency task, where subjects generated verbs based on concrete nouns they were given, also found that the left hemisphere was dominant during a language task. ${ }^{[35]}$ They also found that the left hemisphere was dominant during a resting state condition. ${ }^{[35]}$ These results do not concur with the results found in the current study during the rest condition. This may be explained by the fact that during both rest conditions (of the Word Expression Paradigm,) subjects were thinking about the previous verbal fluency tasks given to them. This was confirmed after the study when subjects were asked to describe what they thought about during their rest period. All subjects confirmed that they were thinking of previous responses given during the verbal fluency task, and other responses that they could have given. 


\subsubsection{1 -Regional Activation Analysis}

As previously mentioned in section 3.4.3 the regional analysis looks at four distinct areas in the fronto-temporal region of the brain. These areas include the left anterior (LA) (encompassing Broca's area), the left posterior (LP) (encompassing Wernicke's area), and the right anterior (RA), and the right posterior (RP) of the fronto-temporal region of the brain.

Figure 12 provides the regional averaged activation plots (in terms of $\mathrm{HbO}$ (red), $\mathrm{HbR}$ (blue), and $\mathrm{HbT}($ black)) for the four regions in the left and right fronto-temporal regions obtained in response to the word expression paradigm. The results from the regional analysis are similar to those obtained during the channel-wise activation analysis for the word expression paradigm. Observing the activation in the left anterior region (encompassing left Broca's area) HbO shows an increase in amount of activation, while HbR shows a decrease. 


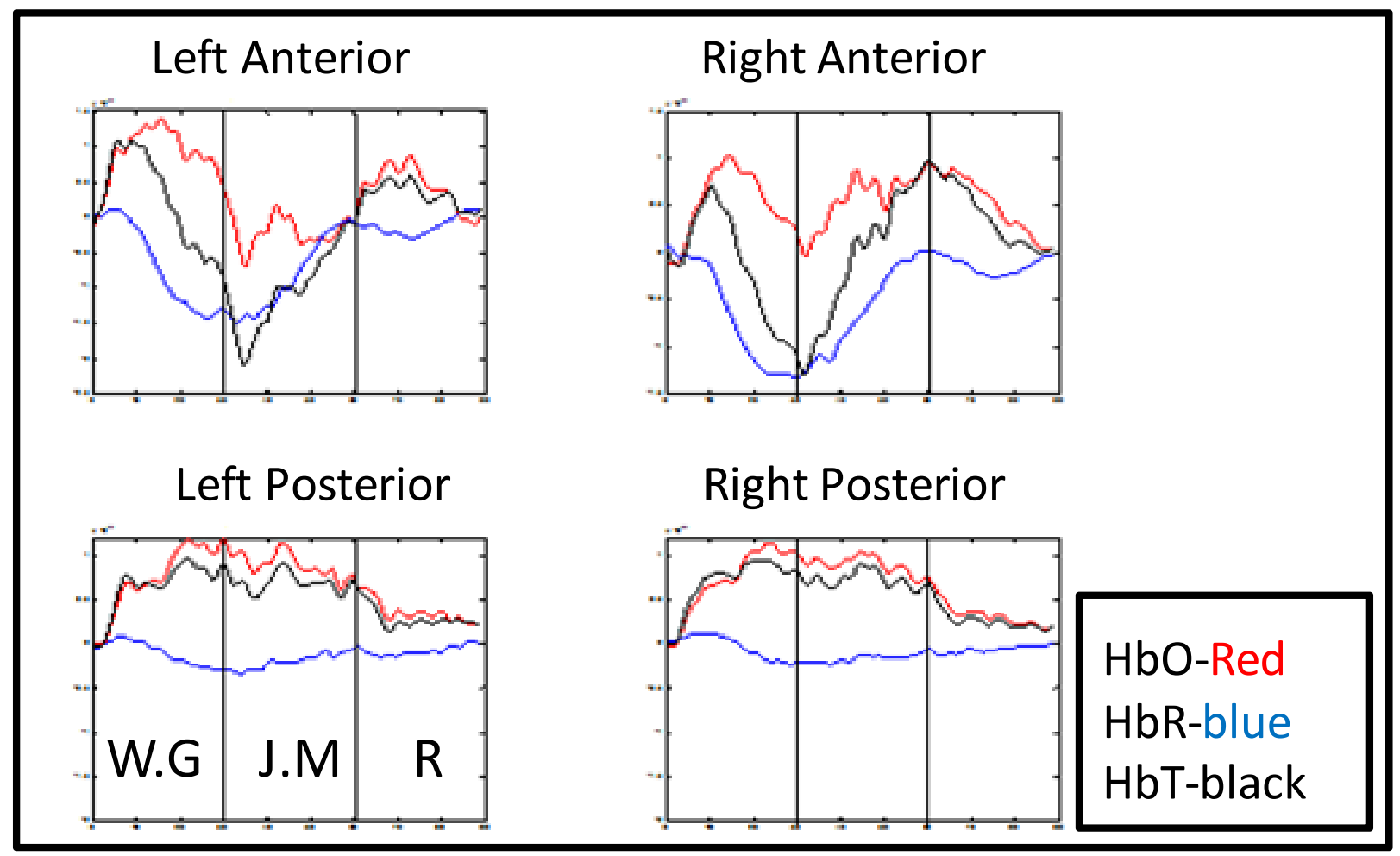

Figure 12. Regional Word Expression Paradigm activation plots. The plots show oxygenated (red) and deoxygenated (blue) hemoglobin activation in the left and right temporal regions of the brain during the Word Expression Paradigm. All plots are shown on the same scale. The y-axis corresponds to the relative change in hemoglobin, while time is shown on the $\mathrm{x}$-axis. The plots are divided into the three 30 second stimuli (from left to right), Word Generation (W.G), Jaw Movement (J.M), Rest (R). The y-axis represents the relative concentration change in hemoglobin species (abu). The x-axis represents time in seconds. All plots are set to the same scale, with the $y$-scale ranging from $\left(-2 \times 10^{-5}-2 \times 10^{-5}\right)$.

This trend can also be seen in the right anterior fronto-temporal region (encompassing Broca's area), as well as in the posterior regions (both left and right, encompassing Wernicke's area, as well as its right hemisphere analog) during the word generation task. During the jaw movement task there is a decrease in the overall mean amplitude of activation for both $\mathrm{HbO}$ and 
$\mathrm{HbR}$ across all four regions. The activation pattern is similar to the word generation task, where $\mathrm{HbO}$ shows an increase, and $\mathrm{HbR}$ a decrease. The overall activation trend shows a "boxcar" trend previously described in section 4.1.1.

The amplitude of activation (mean, Table 5) is highest for $\mathrm{HbO}$ in the left anterior frontotemporal region (encompassing Broca's area) during the word generation task. For all four regions, the three stimuli are statistically significantly different (i.e. word generation vs. jaw movement, word generation vs. rest, jaw movement vs. rest, $\mathrm{p}<0.05$, for $\mathrm{HbO}, \mathrm{HbR}$, and $\mathrm{HbT}$ as shown in Tables E4-E6 of Appendix E, respectively). The results from the averaged regional activation analysis are the same as those seen during the channel-wise activation analysis during the word expression paradigm.

\begin{tabular}{|c|c|c|c|c|c|c|c|c|c|}
\hline \multicolumn{10}{|c|}{ Word Expression Paradigm Mean Values for each 30 second stimuli } \\
\hline Region & \multicolumn{3}{|c|}{ Word Generation } & \multicolumn{3}{|c|}{ Jaw Movement } & \multicolumn{3}{|c|}{ Rest } \\
\hline & $\mathrm{HbO}$ & $\mathrm{HbR}$ & $\mathrm{HbT}$ & $\mathrm{HbO}$ & $\mathrm{HbR}$ & $\mathrm{HbT}$ & $\mathrm{HbO}$ & $\mathrm{HbR}$ & $\mathrm{HbT}$ \\
\hline Left Anterior & 9.03E-06 & $-6.63 \mathrm{E}-06$ & 66 & $-1.85 \mathrm{E}-06$ & $-8.24 \mathrm{E}-06$ & 05 & 4.26E-06 & $-1.12 \mathrm{E}-06$ & BE-06 \\
\hline Left Po & $7.51 \mathrm{E}-06$ & $-9.49 E-07$ & $6.94 \mathrm{E}-06$ & $8.97 \mathrm{E}-06$ & $-1.99 \mathrm{E}-06$ & 7.03E-06 & $3.67 E-06$ & $-7.36 \mathrm{E}-07$ & $3.16 \mathrm{E}-06$ \\
\hline $\begin{array}{l}\text { Right } \\
\text { Anterior }\end{array}$ & $5.31 \mathrm{E}-06$ & $-6.65 E-06$ & $-1.09 \mathrm{E}-06$ & $5.72 \mathrm{E}-06$ & $-6.08 \mathrm{E}-06$ & $-1.17 E-06$ & $5.13 E-06$ & $-1.28 \mathrm{E}-06$ & 4.24E-06 \\
\hline $\begin{array}{l}\text { Right } \\
\text { Posterior }\end{array}$ & $7.26 \mathrm{E}-06$ & $-2.69 \mathrm{E}-07$ & $6.59 \mathrm{E}-06$ & $8.97 \mathrm{E}-06$ & $-1.64 \mathrm{E}-06$ & 7.37E-06 & $3.30 \mathrm{E}-06$ & $-6.08 \mathrm{E}-07$ & $2.93 \mathrm{E}-06$ \\
\hline
\end{tabular}

Table 5: A table of the mean values for each of the three stimuli across all 4 regions for $\mathrm{HbO}, \mathrm{HbR}$, and $\mathrm{HbT}$ during the Word Expression Paradigm.

In summary the regional activation plots for the word expression paradigm follow trends similar to those observed in the channel-wise activation plots (see figure 7). The highest amplitude of activation was seen for $\mathrm{HbO}$ in the left anterior fronto-temporal region (encompassing Broca's area) during the word generation stimuli. HbO shows a marked increase in the amplitude of activation during all three stimuli, while HbR shows a decrease in the 
amplitude of activation. Amplitude of activation was also lowest during the rest task for $\mathrm{HbO}$, $\mathrm{HbR}$, and $\mathrm{HbT}$ (Table 5). The majority of the regional activation averages were found to not be statistically significantly different from the channel-wise activation data during the word expression paradigm ( i.e. $\mathrm{p}>0.05$, TablesE4-E6 in Appendix E). The only exceptions occurred for the $\mathrm{HbT}$ data between the regional average and channel L7 during both the word generation and jaw movement stimuli, channel L8 during the word generation stimuli, channel R6 during the rest stimuli, and channel R7 during jaw movement. These channels were found to be statistically significantly different from the regional average during these stimuli (i.e. $p<0.05$ ) for $\mathrm{HbT}$ only.

The results from the word expression study show that during the verbal fluency task (word generation task) there is an increase in the relative concentrations of $\mathrm{HbO}$ across all channels, and all four regions. During the verbal fluency task (word generation task) there is also a decrease in the relative concentration of $\mathrm{HbR}$ across all channels, and all four regions. This increase in $\mathrm{HbO}$, and decrease in $\mathrm{HbR}$ signifies that there is a high demand for oxygen by the brain during the generation of words.

\subsubsection{1 -Regional Functional Connectivity Analysis}

Figures 13,14 , and 15 shows the regional functional connectivity results of $\mathrm{HbO}, \mathrm{HbR}$, and $\mathrm{HbT}$, respectively, during the three stimuli in the fronto-temporal regions (left anterior (LA), right anterior (RA), left posterior (LP), right posterior (RP)) of the brain during the word expression paradigm. Observing figure 13, which is an illustration of the functional connectivity during the word expression paradigm for $\mathrm{HbO}$, there is a change in the connectivity during each of the three stimuli. During the word generation task the left vs. right connectivity is very strong, 
both in the anterior fronto-temporal region (encompassing Broca's area), as well as in the posterior fronto-temporal region (encompassing Wernicke's area).

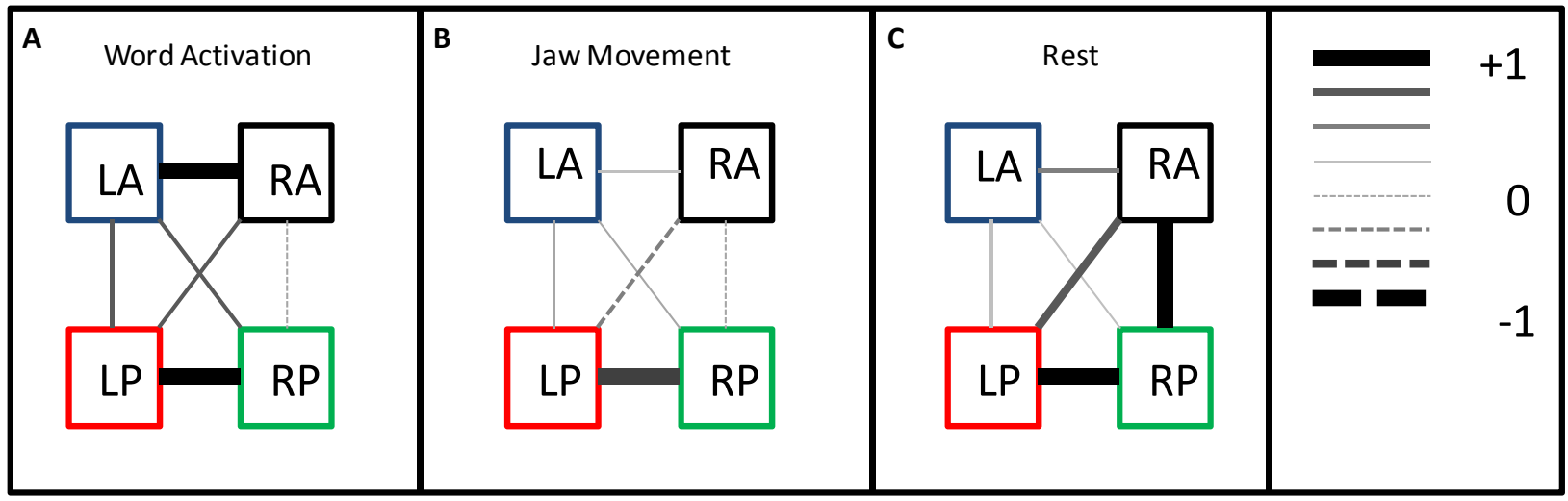

Figure 13. Oxy-hemoglobin Functional Connectivity Diagram. The diagram shows the correlation for the four regions between the left and right hemisphere during the regional Word Expression Paradigm for HbO. The lines are shaded according to the scale on the right hand of the figure, which corresponds to the correlation values calculated for the left and right hemisphere during the Word Expression Paradigm.

There is a moderate correlation between the LA/RA and LP/RP temporal regions, and a weak positive cross correlations between LA vs. RP, and RA vs. LP temporal regions. . During the jaw movement stimuli there is a weak correlation between left and right hemispheres, as well as weak positive and negative cross correlations (LA vs. RP, RA vs. LP) between left and right fronto-temporal regions. During the rest stimuli, the right anterior and posterior temporal regions ( RA vs. RP) (encompassing both right analogs of Broca and Wernicke's areas) show a strong positive correlation, as well as left vs. right posterior fronto-temporal regions (LP vs. RP) ( encompassing Wernicke's area, and its right hemisphere analog).

Observing figure 14, which is an illustration of the functional connectivity during the word expression paradigm for $\mathrm{HbR}$, there is no change in the connectivity during both the word 
activation stimuli, as well as the jaw movement stimuli. During the rest task there is a change in both the left vs. right connectivity (LA vs. RA, LP vs. RP), as well as in the cross correlations (RA vs. LP, LA vs. RP), where the connectivity becomes weaker, and in the right hemisphere it becomes a weak negative correlation (RA vs. RP). Overall, HbR shows strong positive correlations across all four regions, left and right, as well as cross-correlations during both the

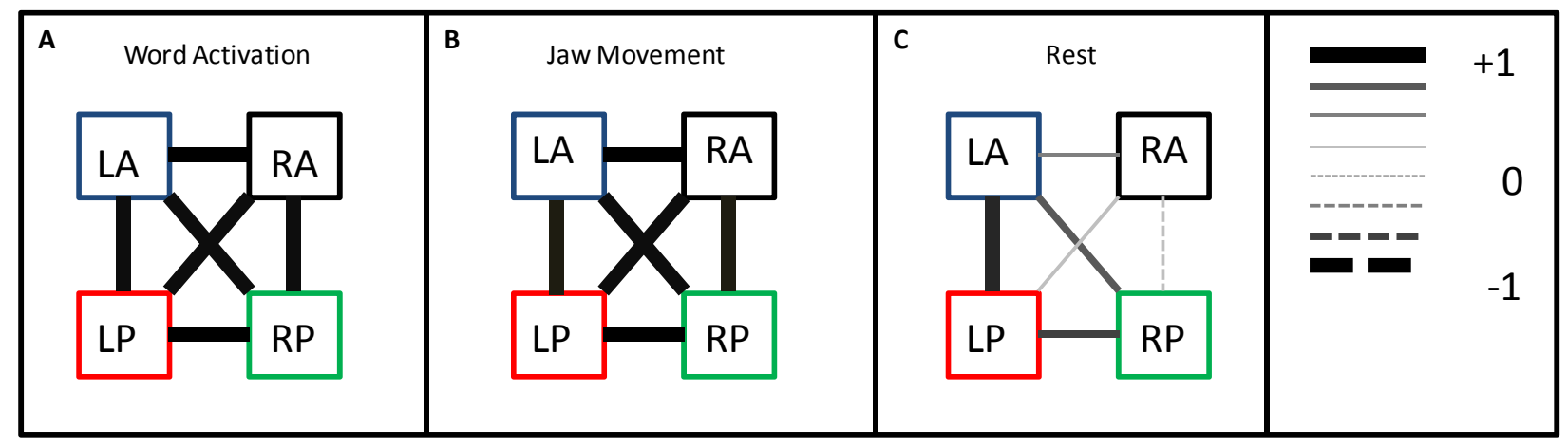

Figure 14. Deoxy-hemoglobin Functional Connectivity Diagram. The diagram shows the correlation for the four regions between the left and right hemisphere during the Word Expression Paradigm for HbR. The lines are shaded according to the scale on the right hand of the figure, which corresponds to the correlation values calculated for the left and right hemisphere during the Word Expression Paradigm.

word generation stimuli, as well as jaw movement stimuli. The connectivity during the rest task is overall weaker and shows more of a random pattern.

The result of $\mathrm{HbT}$ functional connectivity analysis for word expression paradigm is shown in Figure 15 It can be observed from figure 15 which corresponds to functional connectivity during word generation task, that there is a strong functional connectivity or synchrony in the activity between the left anterior region (LA vs. RA) (which encompasses Broca's area and its right homologue) and between the left posterior fronto-temporal region (LP vs. RP), encompassing Wernicke's area and its right analog. 


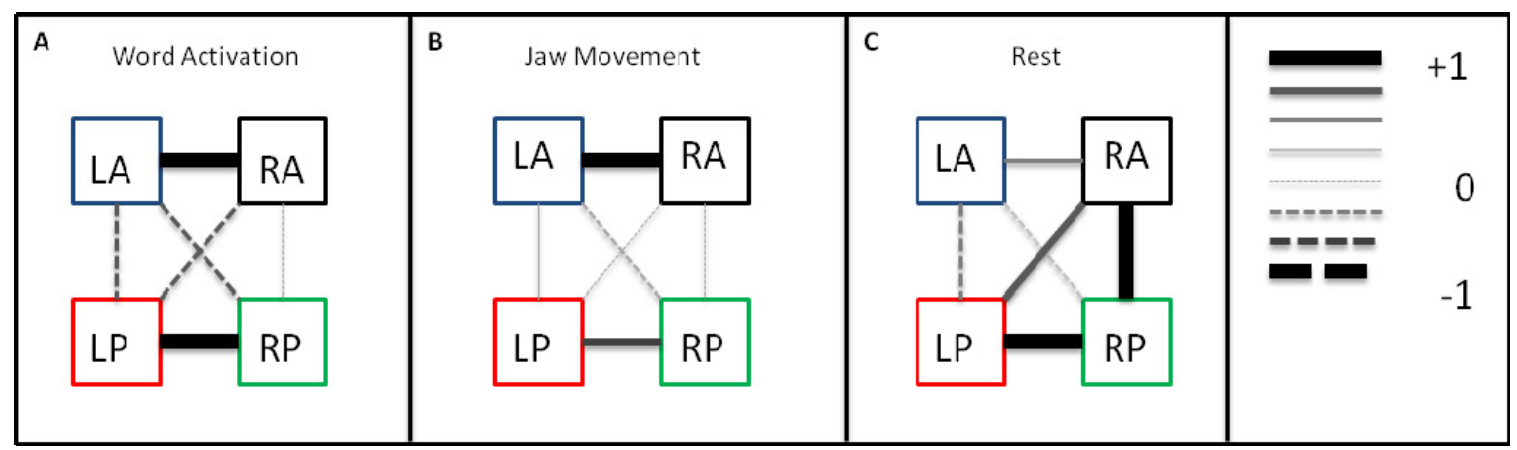

Figure 15.Total Hemoglobin Functional Connectivity Diagram. The diagram shows the correlation for the four regions between the left and right hemisphere during the Word Expression Paradigm for HbT. The lines are shaded according to the scale on the right hand of the figure, which corresponds to the correlation values calculated for the left and right hemisphere during the Word Expression Paradigm.

During jaw movement task strong functional connectivity (as shown in Figure 15) is found between the regions of the brain whose hemodynamic response function is prone to be affected by the jaw movement i.e. the left and right anterior regions. The functional connectivity between left and right posterior regions during jaw movement task weakens as compared to the functional connectivity between these regions during word generation task. During rest task strong functional connectivity can be observed between the right anterior and left posterior but not so between the left anterior and left posterior. Strong functional connectivity was also observed between left and right anterior regions during the rest task.

The connectivity pattern observed for the regional analysis for $\mathrm{HbO}$ resembles the connectivity patterns observed for the channel-wise analysis. During the word activation task, there is a strong correlation between the left and right hemispheres of both the anterior and posterior regions. Observing the jaw movement task, there is a drop in the correlation of both the left and right anterior regions. Observing the rest task, there is a strong correlation between the 
right anterior (RA) and posterior region (RP), as well as between the left (LP) and right (RP) posterior region. These changes between the connectivity patterns across all three tasks suggest that there are again different neuronal pathways being utilized by the brain during a language task, which differ from those taken during both jaw movement and rest tasks. Observing the connectivity patterns for $\mathrm{HbR}$, there is no change in the extent of correlation, or connectivity patterns between the language task, and the jaw movement task. For both the word generation task and jaw movement task all four regions show a high correlation with one another. During the rest task though, there is a drop in the extent of correlation amongst these 4 regions. The neuronal pathways taken by HbR during the rest task differ from those used both in the word generation and jaw movement task, as observed by the changing connectivity patterns of figure 14. The results for $\mathrm{HbT}$ show that both the left and right posterior and anterior regions are highly correlated. Furthermore, during the jaw movement task the anterior region shows a very strong correlation between left and right hemispheres, which is opposite to the results observed for $\mathrm{HbO}$. During the rest task, again the correlations observed between the right temporal regions, and between the right anterior and left posterior regions are similar to those seen for $\mathrm{HbO}$. Across all three stimuli, there are differences in connectivity patterns which suggest again that a different pathway is being taken during language tasks, over jaw movement and rest tasks, but very interestingly is that both $\mathrm{HbO}$ and $\mathrm{HbT}$ show similar connectivity patterns for rest task. This may suggest that while resting and thinking of nothing the same pathways are being utilized. 


\subsubsection{1 -Regional Lateralization Analysis}

Figure 16 depicts the results from the regional cortical lateralization study in the temporal region of the brain during the word expression paradigm.

\begin{tabular}{|c|c|c|c|c|c|c|c|c|}
\hline \multirow[t]{2}{*}{ A } & \multicolumn{2}{|c|}{ Word Generation } & B & \multicolumn{2}{|c|}{ Jaw Movement } & \multirow[t]{3}{*}{ C } & \multicolumn{2}{|c|}{ Rest } \\
\hline & LB & RB & & LB & RB & & LB & $\mathrm{RB}$ \\
\hline & LW & RW & & LW & RW & & LW & RW \\
\hline
\end{tabular}

Figure 16. Lateralization Index Diagram. The following diagram shows the lateralization results for the three stimuli during the regional Word Expression Paradigm in the temporal region. The boxes that are shaded correspond to a dominate region. Those boxes left unshaded correspond to a bilateral lateralization index no dominance).

The results from the regional analysis are similar to those obtained during the channelwise analysis described in section 4.1.3. During the word generation stimuli the left anterior fronto-temporal region (encompassing Broca's area) shows dominance. All other regions during the word generation stimuli show bilateral dominance (i.e. no dominance at all). During the jaw movement stimuli, the dominance shifts to the right hemisphere, where the right anterior frontotemporal region (encompassing the right analog of Broca's area) becomes dominant. During the rest stimuli no region shows dominance, and overall there is bilateral lateralization during the rest task. These results are consistent with the results seen during the channel-wise data (section 4.3.1). 


\subsection{4-Discussion}

During the word expression paradigm the brain becomes activated during the verbal fluency task. The activation plots show that there is an increase in the amount of $\mathrm{HbO}$, with a decrease in HbR across all channels. Furthermore, the highest amplitude of activation occurs in the left anterior region encompassing Broca's area (both channel-wise and regionally). The functional connectivity also shows that during the word expression paradigm there is a strong left vs. right correlation in both the anterior region (encompassing Broca's area and its right analog), as well as in the posterior region (encompassing Wernicke's area and its right analog) for $\mathrm{HbO}$ and $\mathrm{HbR}$, as well as $\mathrm{HbT}$ during the word generation task. Interestingly, there are differences as well in the connectivity patterns across all three stimuli for $\mathrm{HbO}, \mathrm{HbR}$, and $\mathrm{HbT}$, which suggests that during each stimuli there are different neuronal pathways being utilized. Regional functional connectivity results showed strong positive left vs. right correlations, but for $\mathrm{HbO}$ and $\mathrm{HbT}$ there were no real correlations between regions in the same hemisphere, or across hemispheres (i.e. LA vs. RP, and LP vs. RA). Only in HbR are there strong positive correlations across all regions with no change in connectivity patterns. This may suggest that for a verbal fluency task, as well as resting tasks and jaw movements, $\mathrm{HbR}$ is using the same neuronal pathways. Lateralization results suggest that there is more blood in the left hemisphere during the verbal fluency task, namely in the anterior region (LA) (encompassing Broca's area), while during the jaw movement task there is more blood residing in the RA region, as well as during rest (suggested by channelwise data).

Overall, it is believed that during a word generation task, the left anterior region (encompassing Broca's area) plays a major role in the generation of language for right handed 
individuals. The activation results suggest that while the verbal fluency task does cause the brain to increase oxygen supplies, there is very little oxygen consumption occurring during this stimulus. This may be due to the fact that oxygen is supplied faster than it can be depleted (or consumed). Overall the activity of the brain suggests that the task given may be a simple task, which causes minimal exertion in the execution of naming words by the brain. The posterior region also seems to play a role in the generation of language, as suggested by the activation results, as well as previous findings from other groups ${ }^{[37]}$. Although the posterior region (encompassing Wernicke's area and its right analog) follow similar trends to that of the anterior region, the extent (amplitude of activation) and blood flow to the posterior region during a verbal fluency task (from lateralization analysis) is less than that seen in the anterior region.

Results from the word expression show that the left anterior fronto-temporal region (encompassing Broca's area) becomes preferentially activated, and dominant during a verbal fluency task. Results also show that the left posterior fronto-temporal region also becomes activated, yet not to the extent that the anterior region activates. This region encompasses Wernicke's area, an area which was previously thought to be responsible for language reception only. The results from Cannestra et. al(2003), as well as the results from the current study show that while Wernicke is not solely responsible for language expression, it does serve some function during language expression tasks in the temporal region of the brain. ${ }^{[37]}$ 


\section{Chapter 5- Results and Discussion: Word Reception Paradigm}

\section{1- Word Reception Paradigm}

The following chapter describes in detail the results of the word reception paradigm. The activation, functional connectivity, and lateralization for the channel-wise study are first described. Then the regional analysis results from the word reception paradigm in terms of activation, functional connectivity, and lateralization are described. The chapter then focuses on the discussion of the results of both the channel-wise as well as regional analysis. The results from this chapter are based on a language reception paradigm, which is focused on the passive listening of both comprehensible and non-comprehensible speech in the temporal regions of the brain.

5.1.1 -Activation Analysis

Figure 17 provides the activation plots (in terms of $\mathrm{HbO}$ (red), $\mathrm{HbR}$ (blue), and $\mathrm{HbT}$ (black)) for each channel in the left and right fronto-temporal regions obtained in response to the word reception paradigm. 


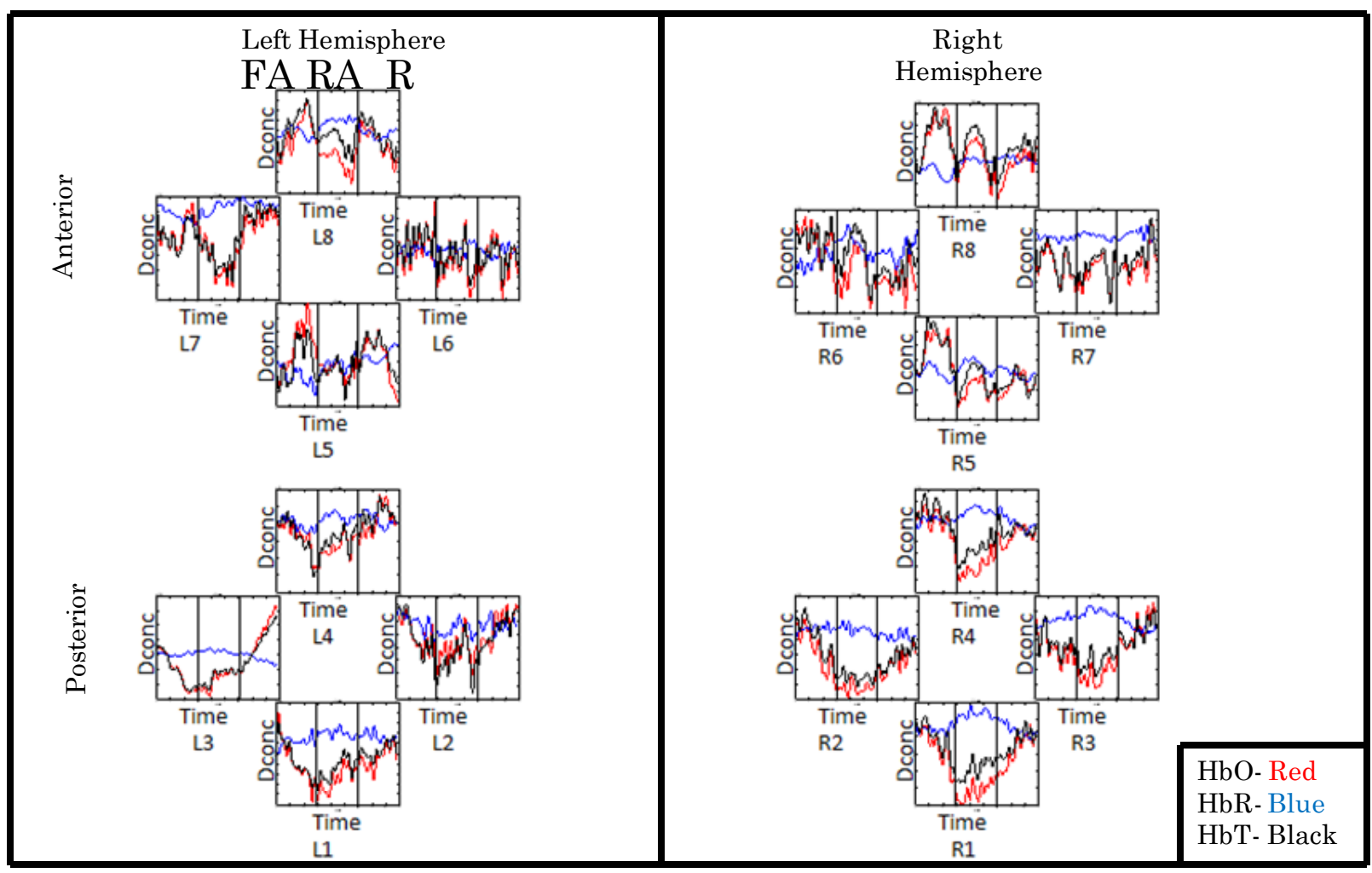

Figure 17. Word Reception Paradigm activation plots. The plots show the oxygenated (red) and deoxygenated (blue) hemoglobin activation in the left and right temporal regions of the brain during the Word Expression Paradigm. All plots are shown on the same scale. The y-axis corresponds to the relative change in hemoglobin, while time is shown on the x-axis. The plots are divided into the three 30 second stimuli (from left to right), Forward Audio, Reverse Audio, Rest. All plots are set to the same scale, with the y scale ranging from $\left(-2 \times 10^{-5}-2 \times 10^{-5}\right)$.

The activation differs across all three stimuli for $(\mathrm{HbO}, \mathrm{HbR}$, and $\mathrm{HbT})$, i.e. $(\mathrm{p}<0.05)$ (Tables F1-F3 of Appendix F). Observing channels 5-8, the activation (mean) tends to be highest during the forward audio tasks (table 6) where the subject is able to listen and comprehend. The activation then seems to drop during the reverse audio task, and returns during rest baseline. Observing channels 1-4 which correspond to Wernicke's area and its right analog, 
the activation pattern seems to initially begin a gradual decrease, with the lowest activation occurring during the reverse audio task, before ascending back to baseline during the rest task.

Noguchi et. al (2002) performed a study where subjects listened to spoken language and were asked to make linguistic decisions with their eyes closed. ${ }^{[59]}$ They performed this study in the Inferior frontal region, as well as the fronto-temporal region. They found that there was high activation in $\mathrm{HbO}$, compared to rest, in the left frontal gyrus, as well as in the left temporal region, but they did not look at the right homologous regions. Observing figure 17, the activation in terms of $\mathrm{HbO}$ is higher than compared to rest in several channels in the left region (Ch.5, Ch.7, Ch.8), but also this trend occurs in the right region (Ch.5, Ch.7, Ch.8).

Brauer et. al (2008) also language performed studies in the perisylvian region, including the superior temporal cortex, and the inferior frontal cortex. ${ }^{[40]}$ Subjects from this study were asked to listen to short spoken sentences, with an average length of 2 seconds. They observed activation in the anterior fronto-temporal region (which included Broca's area and its right analog) during the stimuli, but also observed bilateral activation in the inferior frontal and superior temporal cortices. They concluded that for semantic language processing, Broca's region is primarily involved. Observing Channels L6 and R6 from the word expression activation study, there is a bilateral activation in the superior temporal cortices. Brauer also reported seeing bilateral activation in these locations. Bilateral activation is where the mean from an activation task is compared to the control rest task, in the same location or channel, in both the left and right hemisphere. But bilateral activation does not just occur in the superior temporal cortices, but across all channels of the fronto-temporal region during a word reception paradigm, when comparing our activation tasks to our rest (control) task (see Table 6). 
Sato et. al (1999) chose to present a dichotic listening paradigm, where subjects were asked to track targets across three stimuli, one being just an audio tone, the other a repeat sentence, and finally listening to a repeat sentence while listening to a story. ${ }^{[36]}$ They observed both the left and right temporal cortices. They found that the activity for the story task was localized in the left superior temporal cortices. They also found that $\mathrm{HbO}$ was much higher for the story task, when compared to the repeat task. The story task also showed a marked increase in $\mathrm{HbO}$, and a decrease in $\mathrm{HbR}$. Looking at the results in Figure 17 for the word reception paradigm, in channels (L8, L5, R6, R8, R5) during the forward audio task, there is an increase in the concentration of $\mathrm{HbO}$, and a decrease in the concentration of HbR. For all the channels corresponding to the anterior fronto-temporal region (Ch.5-Ch.8), the highest activation during the word reception paradigm occurred in the forward audio task (Table 6). Table 6 shows the mean for all of the channels for each of the three stimuli (Forward Audio, Reverse Audio, and Rest) for $\mathrm{HbO}, \mathrm{HbR}$, and $\mathrm{HbT}$.

Nakai et. al (1999) used a listening comprehension paradigm of sentences, both in the subject's native language, and in a non-native language. ${ }^{[60]}$ They monitored both Broca's region and Wernicke's region during the paradigm. The results showed that Wernicke's region became activated during all three stimuli. Furthermore, they found that the activation was nearly identical for the native language, when compared to the non-native language, but activation was slightly higher during the native language. Observing Figure 17 which illustrates the activation results from the word reception paradigm shows that Wernicke's region does become activated for both the forward audio stimuli and the reverse audio stimuli across both the left and right hemispheres when comparing them to the control rest task. 


\begin{tabular}{|c|c|c|c|c|c|c|c|c|c|}
\hline \multicolumn{10}{|c|}{ Word Reception Paradigm Mean Values for each 30 second stimuli } \\
\hline \multirow[t]{2}{*}{ Channel } & \multicolumn{3}{|c|}{ FA } & \multicolumn{3}{|c|}{ RA } & \multicolumn{3}{|c|}{ Rest } \\
\hline & $\mathrm{HbO}$ & $\mathrm{HbR}$ & $\mathrm{HbT}$ & $\mathrm{HbO}$ & $\mathrm{HbR}$ & $\mathrm{HbT}$ & $\mathrm{HbO}$ & $\mathrm{HbR}$ & $\mathrm{HbT}$ \\
\hline L1 & $-1.8 \mathrm{E}-06$ & $1.89 \mathrm{E}-07$ & $-1.7 \mathrm{E}-06$ & $-3 E-06$ & 9.84E-07 & $-2.4 \mathrm{E}-06$ & $-1.4 \mathrm{E}-06$ & $6.63 \mathrm{E}-07$ & $-7.1 \mathrm{E}-07$ \\
\hline L2 & $-1 \mathrm{E}-06$ & $-8.3 \mathrm{E}-08$ & $-1.1 \mathrm{E}-06$ & $-2.2 \mathrm{E}-06$ & $-6.5 \mathrm{E}-07$ & $-2.9 \mathrm{E}-06$ & $-4.6 \mathrm{E}-07$ & $-2.8 \mathrm{E}-07$ & $-7.4 \mathrm{E}-07$ \\
\hline L3 & $-2.3 \mathrm{E}-06$ & $6.33 \mathrm{E}-08$ & $-2.2 \mathrm{E}-06$ & $-3.4 \mathrm{E}-06$ & $4.4 \mathrm{E}-07$ & $-3 \mathrm{E}-06$ & $1.96 \mathrm{E}-06$ & $-5.6 \mathrm{E}-07$ & $1.39 \mathrm{E}-06$ \\
\hline L4 & $-7.3 \mathrm{E}-07$ & $-6.1 \mathrm{E}-08$ & $-7.9 \mathrm{E}-07$ & $-1.4 \mathrm{E}-06$ & 3.87E-07 & $-9.6 \mathrm{E}-07$ & $1.83 \mathrm{E}-07$ & $2.33 \mathrm{E}-07$ & 4.16E-07 \\
\hline L5 & 1.07E-06 & $-1 \mathrm{E}-06$ & $4.38 \mathrm{E}-08$ & $-4.2 \mathrm{E}-07$ & $-1.7 \mathrm{E}-07$ & $-5.9 E-07$ & $2.7 \mathrm{E}-07$ & $3.8 \mathrm{E}-07$ & $6.51 \mathrm{E}-07$ \\
\hline L6 & $1.71 \mathrm{E}-06$ & $4.65 \mathrm{E}-07$ & $2.18 \mathrm{E}-06$ & $-1.7 \mathrm{E}-06$ & $7.85 \mathrm{E}-07$ & $-9.1 \mathrm{E}-07$ & $-7.7 \mathrm{E}-07$ & $2.94 \mathrm{E}-07$ & $-4.7 \mathrm{E}-07$ \\
\hline L7 & $-1.4 \mathrm{E}-06$ & $-2.8 \mathrm{E}-07$ & $-1.7 \mathrm{E}-06$ & $-3.4 \mathrm{E}-06$ & 2.43E-07 & $-3.1 \mathrm{E}-06$ & $-7.8 \mathrm{E}-07$ & 5.13E-07 & $-2.6 \mathrm{E}-07$ \\
\hline L8 & $7.58 \mathrm{E}-07$ & 8.03E-07 & $1.56 \mathrm{E}-06$ & $-1.8 \mathrm{E}-06$ & 1.99E-06 & 1.74E-07 & $-1.2 \mathrm{E}-07$ & $7.31 \mathrm{E}-07$ & $6.13 E-07$ \\
\hline R1 & $-5.9 E-07$ & $4.12 \mathrm{E}-07$ & $-1.8 \mathrm{E}-07$ & $-3.2 \mathrm{E}-06$ & $1.31 \mathrm{E}-06$ & $-1.9 \mathrm{E}-06$ & $-7.9 \mathrm{E}-07$ & $4.25 \mathrm{E}-07$ & $-3.6 \mathrm{E}-07$ \\
\hline $\mathrm{R} 2$ & $-6.6 \mathrm{E}-07$ & $7.69 \mathrm{E}-07$ & $1.13 \mathrm{E}-07$ & $-3.2 \mathrm{E}-06$ & $7.32 \mathrm{E}-07$ & $-2.5 \mathrm{E}-06$ & $-1.5 \mathrm{E}-06$ & $2.6 \mathrm{E}-07$ & $-1.2 \mathrm{E}-06$ \\
\hline R3 & $-7.8 \mathrm{E}-07$ & $1.66 \mathrm{E}-07$ & $-6.1 \mathrm{E}-07$ & $-1.7 \mathrm{E}-06$ & 4.44E-07 & $-1.2 \mathrm{E}-06$ & $-2.3 \mathrm{E}-07$ & $-4.7 E-08$ & $-2.7 \mathrm{E}-07$ \\
\hline R4 & 4.79E-07 & $5.8 \mathrm{E}-07$ & $1.06 \mathrm{E}-06$ & $-3 E-06$ & $1.28 \mathrm{E}-06$ & $-1.7 \mathrm{E}-06$ & $-5.8 \mathrm{E}-07$ & $4.43 \mathrm{E}-07$ & $-1.4 \mathrm{E}-07$ \\
\hline R5 & $1.75 \mathrm{E}-06$ & $1.46 \mathrm{E}-07$ & $1.9 \mathrm{E}-06$ & $-9.8 \mathrm{E}-07$ & $6.3 \mathrm{E}-07$ & $-3.5 \mathrm{E}-07$ & $-6.4 \mathrm{E}-07$ & $8.16 \mathrm{E}-08$ & $-5.5 E-07$ \\
\hline R6 & $7.36 \mathrm{E}-07$ & $9.6 \mathrm{E}-08$ & $8.32 \mathrm{E}-07$ & $-4.3 \mathrm{E}-07$ & $6.3 \mathrm{E}-07$ & $2.02 \mathrm{E}-07$ & $-7.3 \mathrm{E}-07$ & $3.65 \mathrm{E}-07$ & $-3.7 \mathrm{E}-07$ \\
\hline R7 & $-2.3 \mathrm{E}-06$ & $2.43 \mathrm{E}-07$ & $-2 E-06$ & $-3 E-06$ & 4.47E-07 & $-2.6 \mathrm{E}-06$ & $-2.1 \mathrm{E}-06$ & $5.5 \mathrm{E}-07$ & $-1.6 \mathrm{E}-06$ \\
\hline R8 & 3.09E-06 & $-2.9 \mathrm{E}-08$ & $3.06 \mathrm{E}-06$ & $9.79 \mathrm{E}-07$ & $9.66 \mathrm{E}-07$ & $1.95 \mathrm{E}-06$ & $5.02 \mathrm{E}-07$ & $1.13 \mathrm{E}-06$ & $1.64 \mathrm{E}-06$ \\
\hline
\end{tabular}

Table 6: A table of the mean values for each of the three stimuli across all 16 channels for $\mathrm{HbO}, \mathrm{HbR}$, and $\mathrm{HbT}$ during the Word Reception Paradigm.

During the word reception paradigm there is an increase in the concentration of $\mathrm{HbR}$, and a decrease in the concentration of $\mathrm{HbO}$ during both the forward audio task, and reverse audio task (of the word reception paradigm) in the posterior region (encompassing Wernicke's area and its right analog). This suggests that during a receptive task the brain, while demanding oxygen ( $\mathrm{HbO}$ ), the brain is actually consuming the oxygen supply faster than it can be supplied. This possibly suggests that during receptive tasks the brain is consuming some of its oxygen supplies to comprehend what is being spoken. This becomes more evident in the reverse audio task, where the concentration of $\mathrm{HbR}$ is highest, and the concentration of $\mathrm{HbO}$ is lowest. During this task, the passage being spoken was designed not to be understood, since it was read from last 
word to the first word. The activation trend shows that in the posterior region there is a larger increase in the concentration of $\mathrm{HbR}$, and a large decrease in the concentration of $\mathrm{HbO}$ present. This suggests that while the brain works hard (than at rest) to understand something it can comprehend, it works even harder to try and understand something it cannot comprehend, as evident by the large consumption of $\mathrm{HbO}$ during these tasks.

\subsection{2 -Functional Connectivity Analysis}

Figure 18 shows the (left $v s$. right) functional connectivity results of $\mathrm{HbO}$ for the three stimuli in the fronto-temporal regions of the brain during the word reception paradigm. Observing channels 5-8 which correspond to the anterior fronto-temporal region (encompassing Broca's area and its right analog), there are no differences in the correlations observed for both the forward audio stimuli, and the reverse audio stimuli.

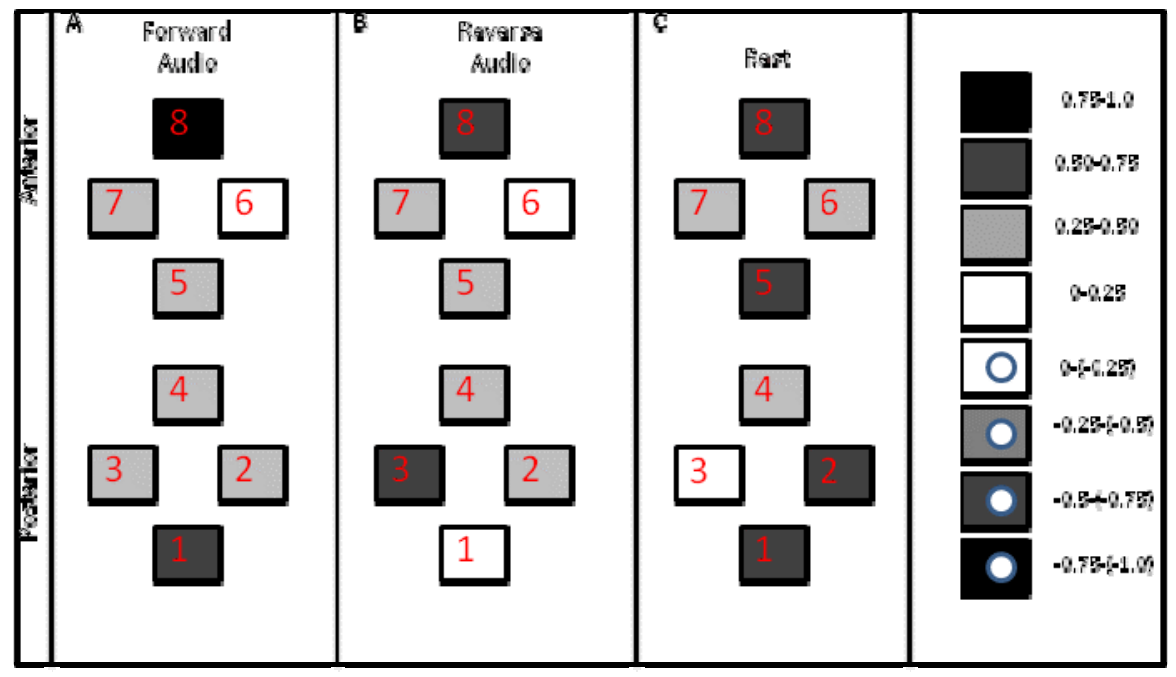

Figure 18. Oxy-hemoglobin Functional Connectivity Diagram. The diagram shows the correlation for channels 1-8 between the left and right hemisphere during the Word Reception Paradigm for HbO. The boxes are shaded according to the scale on the right hand of the figure, which corresponds to the correlation values calculated for the left and right hemisphere during the Word Reception Paradigm. 
When comparing the connectivity patterns of both the forward and reverse audio stimuli w.r.t rest stimuli, there are differences in all the channels, except channel 7. When comparing the connectivity patterns in channels 1-4 for either the forward audio stimuli or the reverse audio stimuli, to one another or to the rest stimuli, there are differences in the connectivity patterns. Wernicke's area seems to be more sensitive to these stimuli than Broca's area as observed from the change in connectivity patterns.

Figure 19 shows the (left $v s$. right) functional connectivity results of HbR for the three stimuli in the temporal regions of the brain during the word reception paradigm. Observing channels 5-8, which correspond to the anterior fronto-temporal region (encompassing Broca's area and its right analog) there are no changes when comparing forward audio stimuli connectivity patterns to reverse audio stimuli connectivity patterns. But when comparing either of these two connectivity patterns to that obtained during the rest stimuli there are differences. Observing channels 1-4, which correspond to the posterior fronto-temporal region (encompassing Wernicke's area and its right analog), there are insignificant changes when comparing the connectivity patterns of the forward audio stimuli vs. reverse audio stimuli or rest stimuli. 


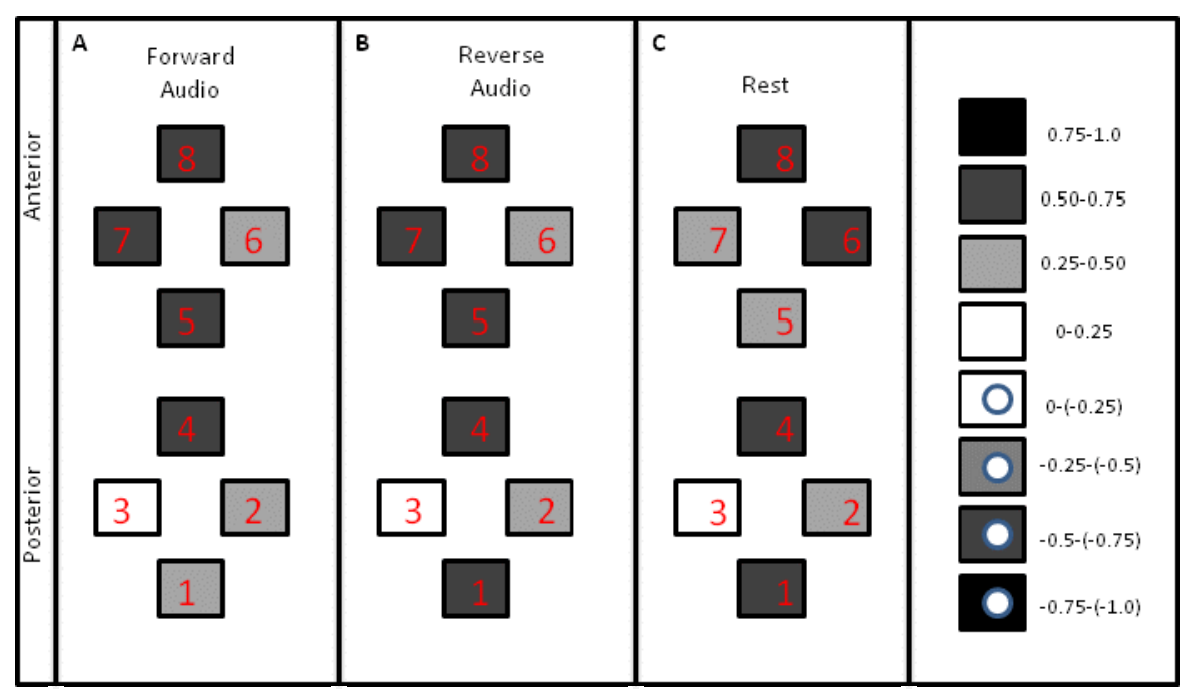

Figure 19. Deoxy-hemoglobin Functional Connectivity Diagram. The diagram shows the correlation for channels 1-8 between the left and right hemisphere during the Word Reception Paradigm for HbR. The boxes are shaded according to the scale on the right hand of the figure, which corresponds to the correlation values calculated for the left and right hemisphere during the Word Reception Paradigm.

Figure 20 shows the (left vs. right) functional connectivity results of $\mathrm{HbT}$ for the three stimuli in the fronto-temporal regions of the brain during the word reception paradigm. Observing channels 5-8, which correspond to the anterior fronto-temporal region, there are changes in the connectivity patterns between the forward audio, reverse audio, and rest stimuli. Observing channels 1-4, which correspond to the posterior fronto-temporal region, the only difference in the connectivity pattern comes from reverse audio stimuli. The connectivity pattern observed during the forward audio stimuli and rest stimuli are similar for these channels. 


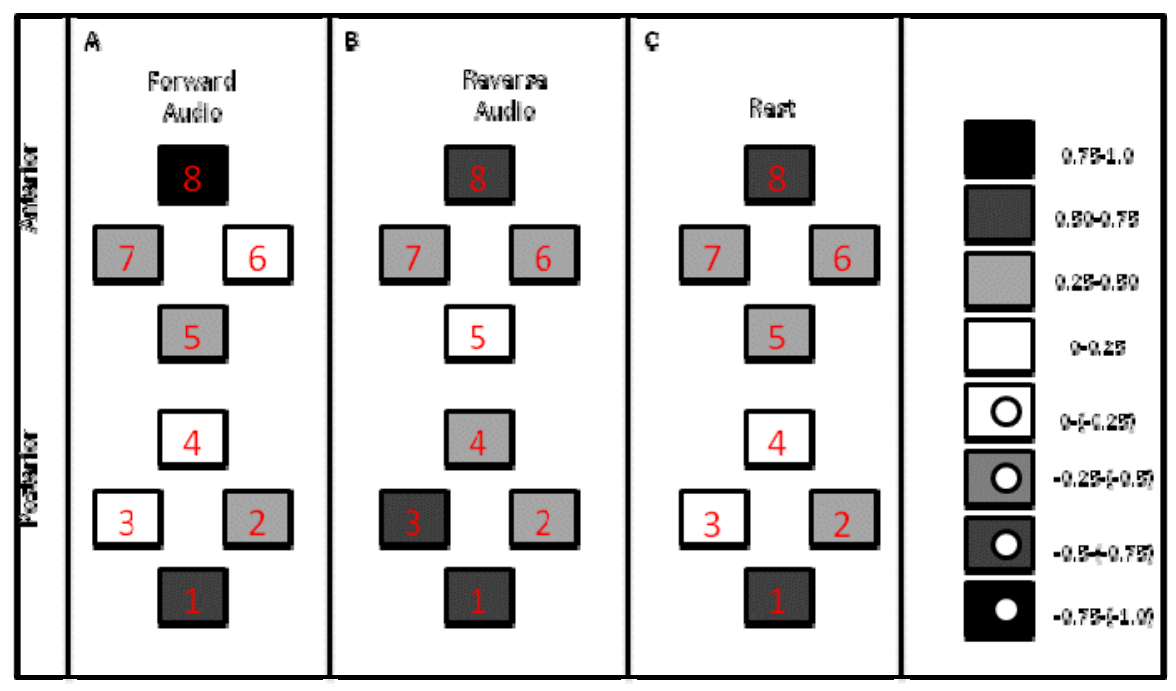

Figure 20. Total hemoglobin Functional Connectivity Diagram. The diagram shows the correlation for channels 1-8 between the left and right hemisphere during the Word Reception Paradigm for HbT. The boxes are shaded according to the scale on the right hand of the figure, which corresponds to the correlation values calculated for the left and right hemisphere during the Word Reception Paradigm.

The results from the word reception functional connectivity analysis correlate to similar studies performed using MRI by Hampson et. al (2002). ${ }^{[47]}$ In their study, subjects were asked to listen to a story being narrated to them, with several rest periods during the story. The results showed that there were correlations between both Broca's regions and Wernicke's regions during both the rest task, as well as during the story task. Further analysis showed that the correlations were significantly higher during the story task compared to rest task in these regions. ${ }^{[47]}$

In summary, the connectivity patterns change in both the anterior fronto-temporal region, and the posterior fronto-temporal region during both language stimuli (forward audio, reverse audio) when comparing to rest stimuli. The changes in connectivity during the word reception paradigm are unlike the changes observed in the word expression paradigm where only the anterior fronto-temporal region showed changes in connectivity. Observing $\mathrm{HbO}$, there is no 
change in the connectivity patterns in either the anterior or posterior region during the forward audio and reverse audio task. This suggests that during both a language reception paradigm that is comprehensible, and one that is not, there is no difference in pathways taken by the brain in the temporal regions. The pattern does change during the rest task, which suggests that a new pathway is being taken when the brain is at rest (i.e. not thinking of anything).

Observing figure 19, which depicts the correlation patterns for HbR during the word reception pattern there is no change in the connectivity pattern in both the anterior and posterior region during both the forward and reverse audio task. The only change in the pattern occurs during rest task in the anterior fronto-temporal region, but in the posterior region again there is no change except channel 1 . This lack of difference in the connectivity pattern suggests that there is no real change in the pathways taken by either the anterior fronto-temporal or posterior frontotemporal regions while listening to either comprehensible or non-comprehensible speech, the same areas stay correlated to one another, and the same pathways are utilized by the brain (both for $\mathrm{HbO}$ and $\mathrm{HbR})$.

Observing figure 20, which illustrates the connectivity of $\mathrm{HbT}$ across all three stimuli during the word reception paradigm, there are changes in the connectivity patterns in the anterior region between forward audio and reverse audio tasks, but there is no difference between the reverse audio task and rest task. In the posterior region, the connectivity pattern is the same during both the forward audio task, and rest task, but different during the reverse audio task. This suggests that in the anterior region there are no differences in the pathway taken between the reverse audio task and the rest task, but during the forward audio task there is a different pathway being utilized. On the contrary in the posterior region, the same pathways are being utilized 
during the forward audio task, and rest task, but when the language becomes noncomprehensible during the reverse audio task, a new pathway is chosen by the brain in the posterior region, and then goes back to the pathways previously used in the forward audio task during the rest task.

\subsection{3 -Lateralization Analysis}

Figure 21 shows the results from the cortical lateralization study in the fronto-temporal region of the brain during the word reception paradigm. During the forward audio task, both the anterior fronto-temporal region and the posterior fronto-temporal region show dominance in the right hemisphere, with right posterior (encompassing the right analog of Wernicke) showing dominance across all channels in this region.

The (left vs. right) amplitude of activation (mean, refer to table 5) is higher across all channels corresponding to the right posterior fronto-temporal region. Since the connectivity of this region is very minimal, and in some cases, there is no connectivity (refer to figure 20), along with the fact that there is a higher activation in the right posterior temporal region leads to cortical dominance in the right posterior fronto-temporal region. 


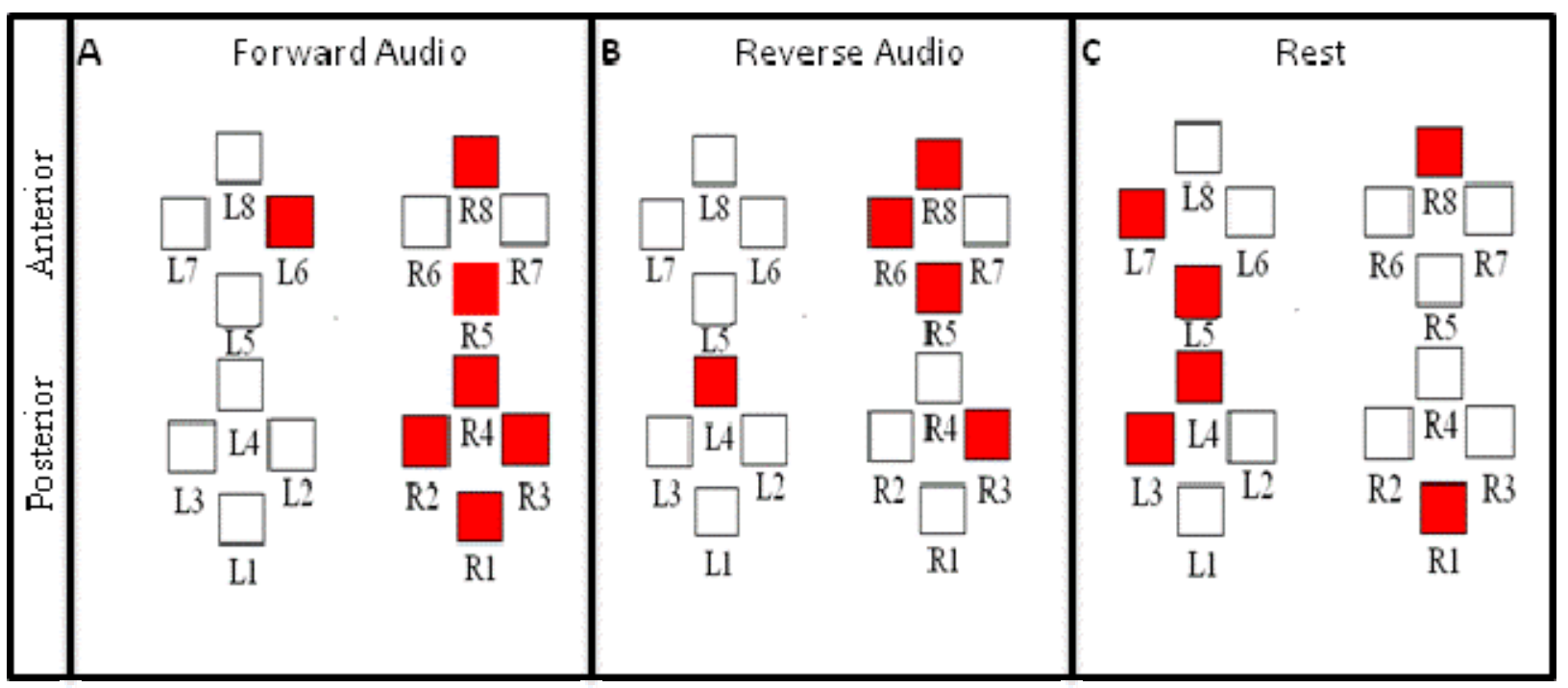

Figure 21. Lateralization Index Diagram. The following diagram shows the lateralization results for the three stimuli during the Word Reception Paradigm in the temporal region. The boxes that are shaded correspond to a dominate channel. Those boxes left unshaded correspond to a bilateral lateralization index( no dominance).

During the reverse audio task the right Broca's region is relatively more dominant than the rest of the temporal region imaged. The dominance during reverse audio randomly occurs in the posterior region, but the right anterior fronto-temporal region is highly dominant, and is due to the fact that the amplitude of activation is much higher in the right region, than the homologous left region ( refer to table6).

Under rest conditions, the left fronto-temporal region was significantly dominant over the right fronto-temporal. These results do not concur from the rest condition results of the word expression paradigm (shown in Figure 15). On the contrary, the word reception paradigm has the rest condition after a jargled reverse audio stimuli, where the brain is possibly truly in rest without any prior influence of jaw movement or thoughts of word generation (as in word expression paradigm). 
These rest condition results coincide with previous results reported by Zhang et. al (2010), who found that during rest conditions, the left hemisphere becomes dominant. ${ }^{[35]}$ Furthermore the results from the current study show that the right hemisphere is dominant both during a passive listening task, as well as a non-comprehensible passive listening task. Lurito et. al (2000) found that passive listening to narrative text induced the least hemispheric lateralization. ${ }^{[52]}$ Furthermore, Caplan et. al (2000) found that listening to recorded stories involves topic following that appears to induce right hemisphere activation. ${ }^{[61]}$ The results from the word reception paradigm are similar to those findings reported by both Caplan et. al (2000) and Lurito et. al (2000), where there is a right hemisphere dominance being seen during passive listening language tasks. Paquette et. al ( 2010) used narrative story tasks (both known and unknown) presented to the subjects both in their native language, and an unknown language. ${ }^{[62]}$ They found during these receptive listening tasks that the left hemisphere shows dominance during a receptive listening task. This finding is opposite to the results seen in the current study, where the right hemisphere was found to be dominant during a receptive listening task.

\subsubsection{1 -Regional Activation Analysis}

Figure 22 represents the activation plots (in terms of $\mathrm{HbO}$ (red), $\mathrm{HbR}$ (blue), and $\mathrm{HbT}$ (black)) for the four regions in the left and right fronto-temporal regions obtained in response to the word reception paradigm. The results from the regional data analysis are the same as the findings in the channel -wise data (as described in Section 5.1.1). 


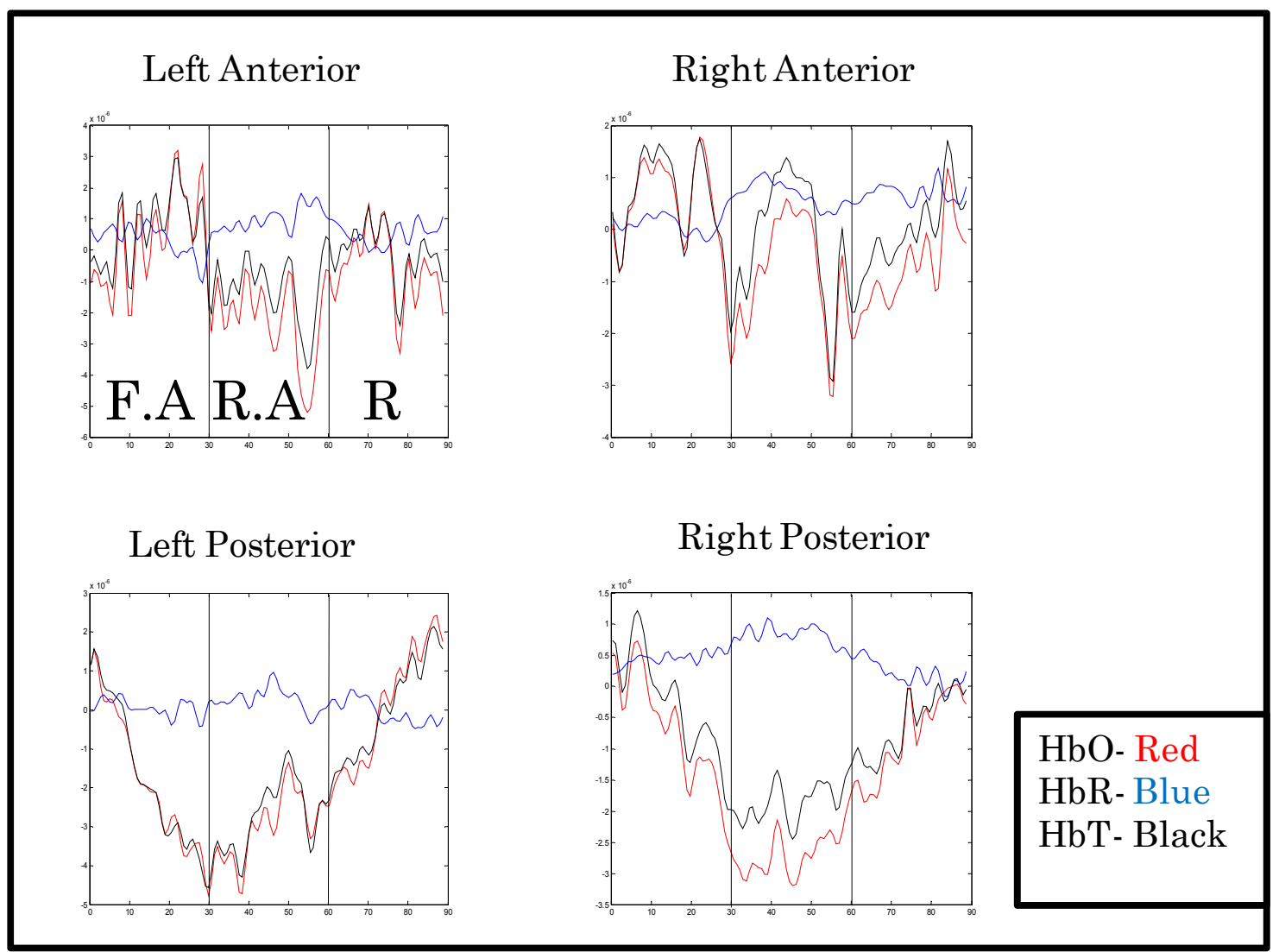

Figure 22. Regional Word Reception Paradigm activation plots. The plots show the oxygenated (red) and deoxygenated (blue) hemoglobin activation in the left and right temporal regions of the brain during the Word Expression Paradigm. All plots are shown on the same scale. The y-axis corresponds to the relative change in hemoglobin, while time is shown on the x-axis. The plots are divided into the three 30 second stimuli (from left to right), Forward Audio (F.A), Reverse Audio (R.A), Rest (R). All plots are set to the same scale, with the y-scale ranging from $\left(-2 \times 10^{-5}-2 \times 10^{-5}\right)$.

The mean obtained in the regional analysis is statistically not significantly different from the means obtained in the channel-wise study during the word reception paradigm $(\mathrm{p}>0.05$, TableF4-F6 in Appendix F). Observing both the left and right anterior regions (encompassing Broca's area, and its right hemisphere analog) there is an increase in activation for $\mathrm{HbO}$, and a 
decrease in activation for $\mathrm{HbR}$ during the forward audio task. Observing the activation in this region during the reverse audio task, there is a decrease in $\mathrm{HbO}$ and an increase in $\mathrm{HbR}$. Observing the posterior regions (both left and right, encompassing Wernicke's area and right analog) there is a decrease in $\mathrm{HbO}$, and an increase in $\mathrm{HbR}$ both during the forward audio task, and reverse audio task. During the reverse audio task there is a larger increase in $\mathrm{HbR}$, and larger decrease in $\mathrm{HbO}$ when compared to the forward audio task, in the same region, as well as compared to the anterior fronto-temporal region (encompassing Broca's area and right analog) as well. The highest amplitude of (mean) activation (as shown in table 7) occurred in the left posterior temporal region (encompassing Wernicke's area) during the reverse audio task for $\mathrm{HbO}$.

\begin{tabular}{|c|c|c|c|c|c|c|c|c|c|}
\hline \multicolumn{10}{|c|}{ Word Expression Paradigm Mean Values for each 30 second stimuli } \\
\hline Region & \multicolumn{3}{|c|}{ Forward Audio } & \multicolumn{3}{|c|}{ Reverse Audio } & \multicolumn{3}{|c|}{ Rest } \\
\hline & $\mathrm{HbO}$ & $\mathrm{HbR}$ & $\mathrm{HbT}$ & $\mathrm{HbO}$ & $\mathrm{HbR}$ & $\mathrm{HbT}$ & $\mathrm{HbO}$ & $\mathrm{HbR}$ & $\mathrm{HbT}$ \\
\hline Left Anterior & $3.12 \mathrm{E}-07$ & $3.24 \mathrm{E}-07$ & $1.66 \mathrm{E}-08$ & $-2.26 \mathrm{E}-06$ & $1.01 \mathrm{E}-06$ & $-3.38 \mathrm{E}-08$ & $-5.50 \mathrm{E}-07$ & $4.99 \mathrm{E}-07$ & $-1.07 \mathrm{E}-09$ \\
\hline $\begin{array}{c}\text { Left } \\
\text { Posterior }\end{array}$ & $-1.80 \mathrm{E}-06$ & $6.01 \mathrm{E}-08$ & $-4.54 \mathrm{E}-08$ & $-3.00 \mathrm{E}-06$ & $2.57 \mathrm{E}-07$ & $-7.18 \mathrm{E}-08$ & $1.08 \mathrm{E}-07$ & $-6.42 \mathrm{E}-08$ & $-4.87 \mathrm{E}-10$ \\
\hline $\begin{array}{c}\text { Right } \\
\text { Anterior }\end{array}$ & $4.41 \mathrm{E}-07$ & $1.16 \mathrm{E}-07$ & $1.45 \mathrm{E}-08$ & $-8.64 \mathrm{E}-07$ & $6.76 \mathrm{E}-07$ & $-3.93 \mathrm{E}-09$ & -7.40E-07 & $6.88 \mathrm{E}-07$ & $-2.43 \mathrm{E}-09$ \\
\hline $\begin{array}{c}\text { Right } \\
\text { Posterior }\end{array}$ & $-7.25 \mathrm{E}-07$ & $4.55 \mathrm{E}-07$ & $-7.04 \mathrm{E}-09$ & $-2.66 \mathrm{E}-06$ & $8.19 \mathrm{E}-07$ & $-4.85 \mathrm{E}-08$ & $-8.00 \mathrm{E}-07$ & 2.07E-07 & $-1.59 \mathrm{E}-08$ \\
\hline
\end{tabular}

Table 7: A table of the mean values for each of the three stimuli across all 4 regions for $\mathrm{HbO}, \mathrm{HbR}$, and $\mathrm{HbT}$ during the Word Reception Paradigm. The red font indicates the region which recorded the largest amplitude of change for both the left and right hemisphere.

The three stimuli are statistically significantly different from one another (i.e. $p<0.05$, forward audio vs. reverse audio, forward audio vs. rest, reverse audio vs. rest) for all four regions and for $\mathrm{HbO}, \mathrm{HbR}$, and $\mathrm{HbT}$ data (tables F4-F6 in Appendix F). These findings are also 
similar to findings from previous groups using receptive language paradigms (as described in section 5.1.1).

\subsubsection{1 -Regional Functional Connectivity Analysis}

Figures 23,24 , and 25 show the regional functional connectivity results of $\mathrm{HbO}, \mathrm{HbR}$, and $\mathrm{HbT}$ during the three stimuli in the fronto-temporal regions of the brain during the word reception paradigm. Observing figure 23 , which is a diagram of the functional connectivity of $\mathrm{HbO}$ during the word reception paradigm, the connectivity pattern differs across all three stimuli. During the forward audio stimuli, there is a very strong positive correlation between the left and right posterior regions.

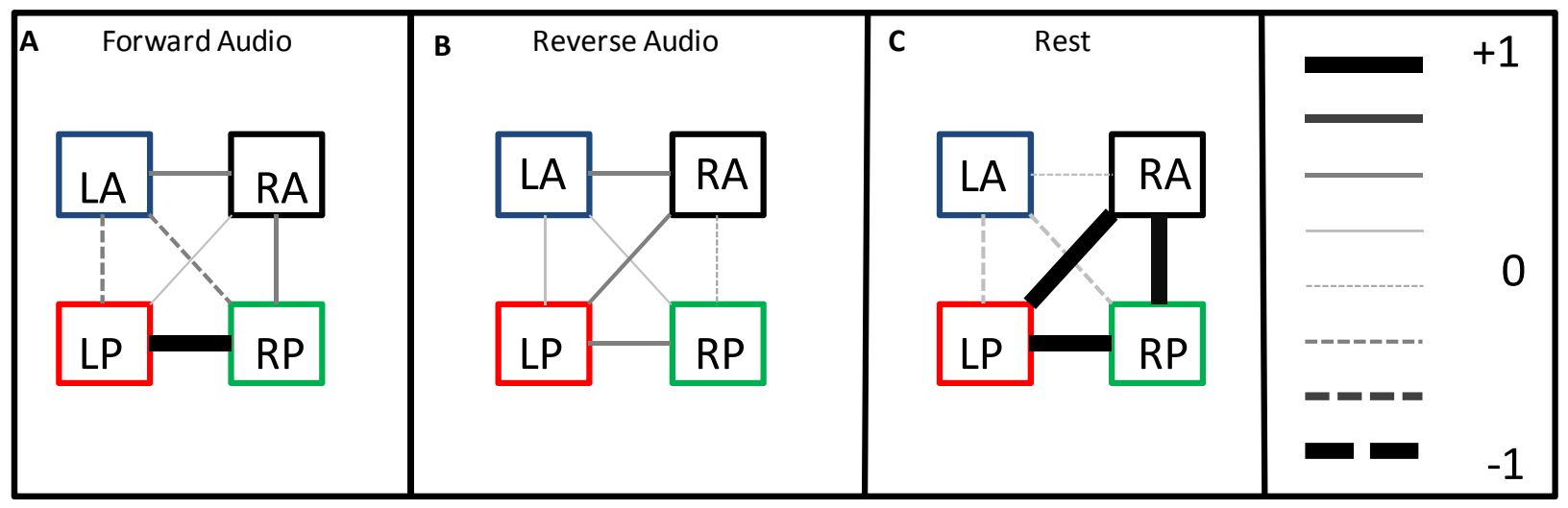

Figure 23. Oxy-hemoglobin Functional Connectivity Diagram. The diagram shows the correlation for the four regions between the left and right hemisphere during the regional Word Reception Paradigm for HbO. The lines are shaded according to the scale on the right hand of the figure, which corresponds to the correlation values calculated for the left and right hemisphere during the regional Word Reception Paradigm.

During the reverse audio stimuli, the connectivity between posterior regions become weaker, but the cross correlations as well as the left anterior (encompassing Broca's area) and the 
left posterior (encompassing Wernicke's area) temporal regions show stronger correlations compared to the forward audio task. The only exception is occurs when comparing RP vs. RA temporal regions, where there is a small negative correlation. When comparing RP vs. RA from the forward audio task, the same two regions showed stronger positive correlations, than during the reverse audio task. During the rest stimuli there are strong correlations between the left and right posterior regions, the left posterior and right anterior, as well as in the right anterior and right posterior regions.

Observing figure 24, which is a diagram of the functional connectivity of HbR during the word reception paradigm, the connectivity pattern differs across all three stimuli. There is a very weak positive correlation during the forward audio task between left and right regions (i.e. LA vs. RA, LP vs. RP), as well as negative correlations between regions (LA vs. RP, RA vs. LP). During the reverse audio stimuli, connectivity in the right hemisphere strengthens, as does the left vs. right posterior fronto-temporal region. In the anterior region, the left vs. right connectivity (i.e. LA vs. RA) becomes negatively correlated during the same task.

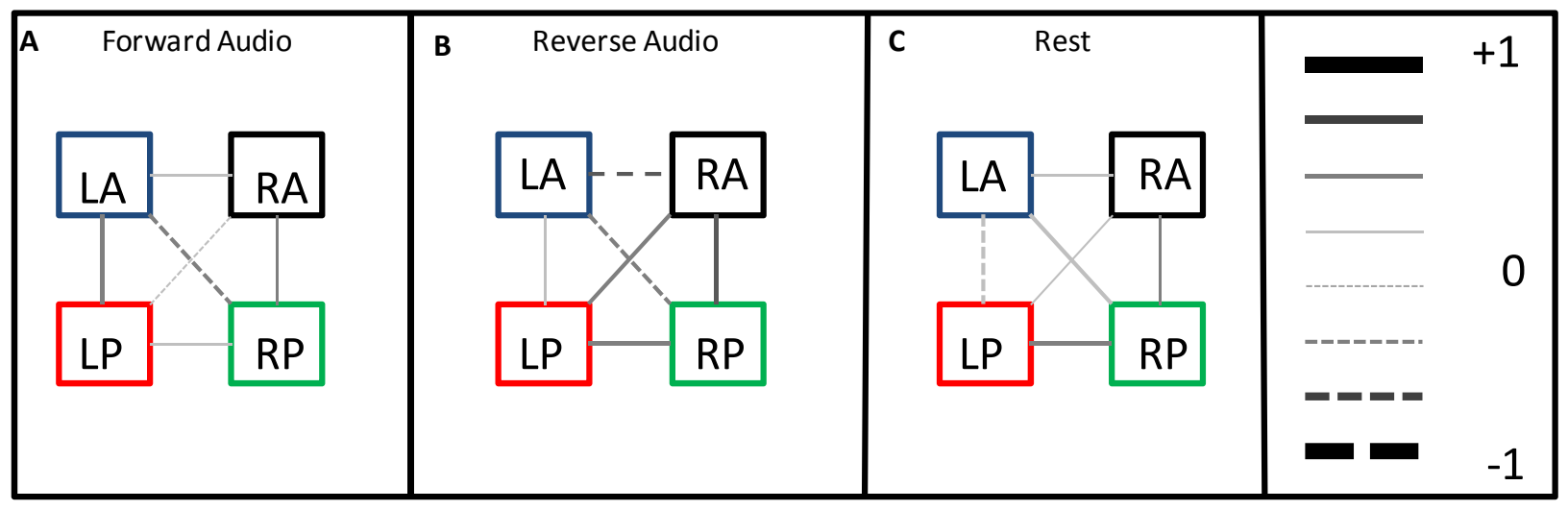

Figure 24. Deoxy-hemoglobin Functional Connectivity Diagram. The diagram shows the correlation for the four regions between the left and right hemisphere during the regional Word Reception Paradigm for HbR. The lines are shaded according to the scale on the right hand of the figure, which corresponds to the correlation values calculated for the left and right hemisphere during the regional Word Reception Paradigm. 
During the rest task, the connectivity pattern shows minimal positive correlations for the majority of the comparisons, with the left hemisphere (anterior vs. posterior, encompassing both Broca's and Wernicke's areas) showing a very weak negative correlation.

Observing figure 25 , which is a diagram of the functional connectivity of $\mathrm{HbT}$ during the word reception paradigm, the connectivity pattern differs across all three stimuli. It can be observed from Figure 25 during forward audio task, that there is a strong functional connectivity or synchrony in the activity only between the left and right posterior fronto-temporal regions. During reverse audio task, weak functional connectivity (as shown in Figure 25) can be observed between all the regions under investigation. The functional connectivity pattern observed during the rest task in this word reception paradigm is similar to that observed during the word generation paradigm (refer to Figure 15 for word expression connectivity pattern for $\mathrm{HbT}$ ).

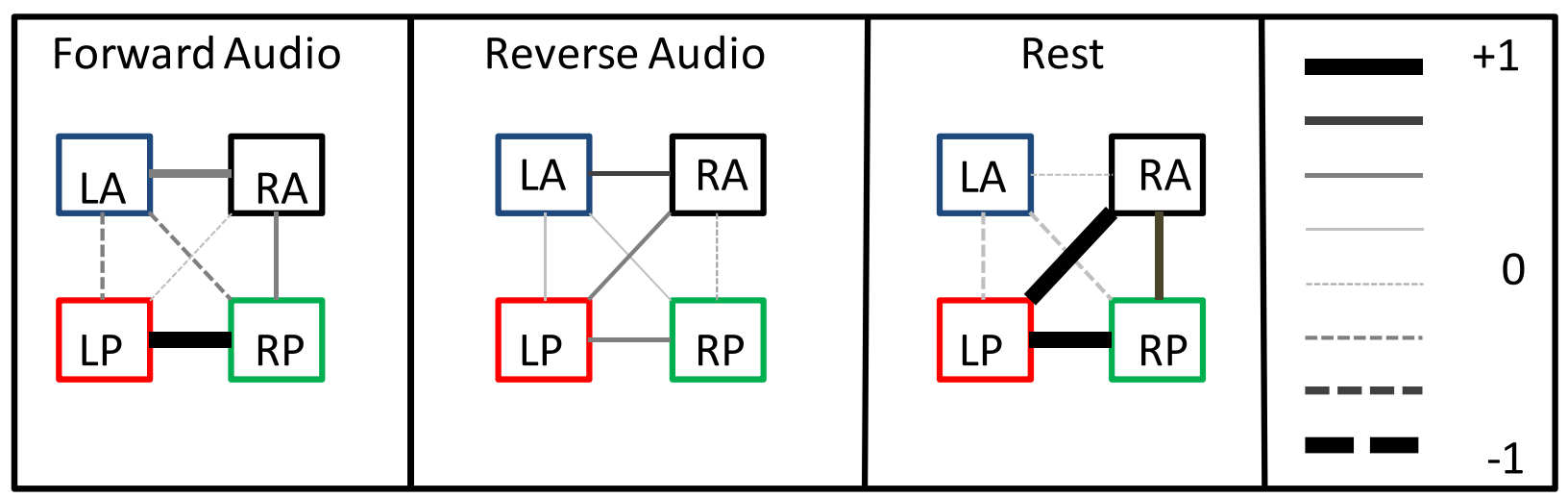

Figure 25. Total hemoglobin Functional Connectivity Diagram. The diagram shows the correlation for the four regions between the left and right hemisphere during the regional Word Reception Paradigm for HbT. The lines are shaded according to the scale on the right hand of the figure, which corresponds to the correlation values calculated for the left and right hemisphere during the regional Word Reception Paradigm. 
Observing the connectivity patterns of $\mathrm{HbO}$ during the word reception paradigm in figure 23, there are differences across all three tasks. Interestingly there is a strong correlation in the posterior fronto-temporal region during the forward audio task. Yet, during the reverse audio task there is a change in the connectivity pattern, as well as a drop in correlation in this region. This suggests that during a forward audio task the brain is taking a different neuronal pathway than during the reverse audio task, and it seems to be more correlated when the language task is one that can be understood (Forward audio), rather than one that is jargled and noncomprehensible (reverse audio). Observing the rest task, again there is a difference in the connectivity pattern observed, when compared to the two language tasks. This change in the connectivity pattern again suggests that there are now new neuronal pathways being taken by the brain during the rest task. Furthermore, observing the rest task, there are strong correlations between the RA vs. RP, between the LP vs. RP, and between the RA vs. LP.

Observing figure 24 , which shows the connectivity patterns for HbR across all three stimuli of the word reception paradigm, there are differences in the connectivity patterns across all three stimuli. Although, there are no strong correlations across any two regions using HbR data.

Observing figure 25 , which depicts the functional connectivity pattern for $\mathrm{HbT}$ during the word reception paradigm, the connectivity results are similar to those of $\mathrm{HbO}$. The left and right posterior fronto-temporal regions show a strong correlation during the forward audio task. There are differences across the connectivity patterns during each stimulus, which again suggests that different pathways (neuronal) are being utilized during each task, independent of one another. Also observing the rest task, the connectivity pattern is similar to that of $\mathrm{HbO}$ during rest task of 
the word reception paradigm, and also similar to the rest pattern observed by $\mathrm{HbO} / \mathrm{HbT}$ during the word expression paradigm. This suggests that when a healthy right handed individual rests, and clears there mind, it seems that the same pathway is being taken regardless of what precluded it, whether it be jargled speech (word reception paradigm), or a jaw movement (word expression paradigm). This is not the case in the channel-wise study. The results seen during the regional analysis may be due to the fact that channels were averaged together to give a general overview of how that region was functioning. Since channels 4 and 5 were excluded, and the remaining channels were averaged together with respect to their given region, some effects seen in the channel-wise study may be cancelled out or otherwise nullified due to the averaging..

The patterns observed during the regional functional connectivity analysis for the word reception paradigm are similar to those results seen during the channel-wise analysis. There are positive left vs. right correlations between anterior and posterior fronto-temporal regions (LA vs. RA, LP vs. RP) observed for both the channel-wise and regional data results. Furthermore, as in the channel-wise study, the regional results show that the connectivity patterns differ across all three stimuli for $\mathrm{HbO}, \mathrm{HbR}$, and $\mathrm{HbT}$. This suggests again that different neuronal pathways are being utilized by the brain during each of the three stimuli. Furthermore even though two of the stimuli are receptive listening tasks, one is comprehensible, while the other task is noncomprehensible, there is still a difference in the connectivity patterns seen in both the channelwise and regional analysis, suggesting that the brain uses different neuronal pathways when listening to comprehensible speech vs. non-comprehensible speech. 


\subsubsection{1 -Regional Lateralization Analysis}

Figure 26 shows the results from the regional cortical lateralization study in the frontofronto-temporal region of the brain during the word reception paradigm. The results from the regional cortical lateralization study are similar to that reported in the channel-wise study for the word reception paradigm (Section 5.1.1). During the forward audio task, the right posterior fronto-temporal region) becomes dominant. During the reverse audio task the dominance shifts from the right posterior fronto-temporal region to the right anterior fronto-temporal region. During the rest task, the left fronto-temporal region becomes dominant, both in the anterior fronto-temporal region, and in the posterior fronto-temporal region.

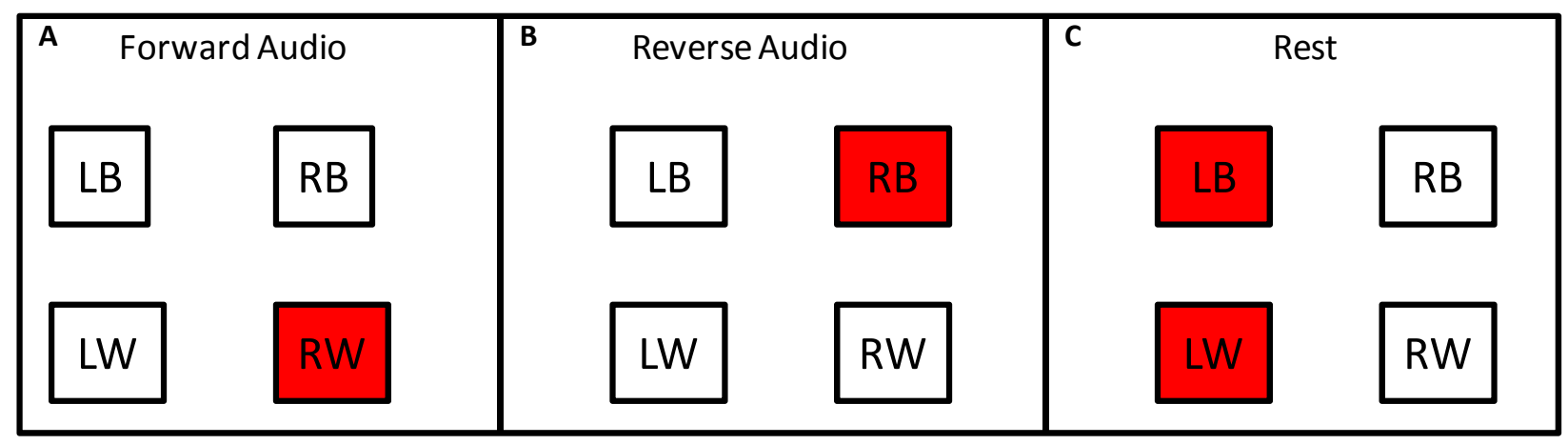

Figure 26. Lateralization Index Diagram. The following diagram shows the lateralization results for the three stimuli during the regional Word Reception Paradigm in the fronto-temporal region. The boxes that are shaded correspond to a dominate region. Those boxes left unshaded correspond to a bilateral lateralization index( no dominance).

\section{2- Discussion}

During the word reception paradigm the brain becomes activated for both receptive language tasks. The trends observed from both the channel-wise and regional activation analysis for word reception paradigm are similar to one another, and opposite to the trend observed in the 
word expression paradigm. During the word expression paradigm, there is an increase in the concentration of $\mathrm{HbO}$, and a decrease in the concentration of $\mathrm{HbR}$ during the verbal fluency task, observed both channel-wise and regionally. During the two receptive language tasks though, there is a decrease in the amount of $\mathrm{HbO}$ and an increase in the amount of $\mathrm{HbR}$ in the posterior region (encompassing Wernicke's area and its right hemisphere analog). The anterior region (encompassing Broca's area and its right hemisphere analog) does not show this trend during the forward audio (comprehensible) task. During the reverse audio task it also shows the same trend of an increase in $\mathrm{HbR}$ and a decrease in $\mathrm{HbO}$. This suggests that for both listening tasks the posterior region is consuming oxygen at a much faster rate than it is supplied to the brain, suggesting that this region is working to try and understand the language being spoken to it (both comprehensible and non-comprehensible). This trend only occurs in the anterior region (encompassing Broca's area and its right hemisphere analog) during the reverse audio task.

Furthermore, the functional connectivity analysis (both channel-wise and regionally)suggests that $\mathrm{HbO}$ shows strong positive correlations (left vs. right) during the forward audio task. During the reverse audio task there is very little correlation between either the left and right hemisphere, or between regions for $\mathrm{HbO}, \mathrm{HbR}$, or $\mathrm{HbT}$. Yet, the functional connectivity results also suggest that for these three tasks the neuronal pathways for $\mathrm{HbO}, \mathrm{HbR}$, and $\mathrm{HbT}$ differ. This implies that when a right handed individual is listening to something understandable, the person uses a different neuronal pathway than when he hears something that is not understandable. Although the activation results suggest that the same trend is occurring in both the left and right hemisphere, the lateralization results both channel-wise and regionally 
suggest that there is more blood flow going into the right hemisphere during both receptive listening tasks.

During the forward audio task (normal speech) the right posterior region (encompassing Wernicke's right hemisphere analog) becomes dominant, and during the reverse audio task the right anterior region (encompassing Broca's right hemisphere analog) becomes dominant. Overall this would suggest that the right hemisphere is seeing more blood during these two tasks, and thus has more involvement in the brain when it comes to receptive listening tasks. During the rest task, there is a shift in dominance, with the left hemisphere seeing more blood flow during this task. This is opposite to the trend observed during the word expression paradigm's rest task, where the right hemisphere becomes dominant. After both paradigms were completed, the subjects were asked to provide their feedback on their typical thoughts during the rest tasks. All the subjects responded that they were thinking of the previous language tasks (either word generation task, during rest period of word expression paradigm, or the forward/reverse audio task, during the word reception paradigm) that they had been given. During the word expression paradigm, subject's responded that they were thinking of other words that they could have responded with. While during the word reception paradigm, subject's said they were thinking of either the first passage they heard and the information it contained, or the second passage they heard and trying to make sense of what they heard. This could explain why the result from each rest task differs from one another, since the subjects were thinking of different things for each task.

Overall, it is believed that for a receptive listening task, the right posterior region (encompassing the right analog of Wernicke's area) is responsible for speech perception in right 
handed individuals. It is also believed that the right anterior region (encompassing the right analog of Broca's area) also has some involvement in the perception of speech during receptive listening tasks.

\section{Chapter 6-Conclusion}

Fifteen healthy normal adults were recruited to participate in a language based functional brain mapping study using NIRS. Subjects were presented with two different language paradigms (Word Expression and Word Reception), in the fronto-temporal regions (specifically Broca's region and Wernicke's region) and the hemodynamic response was measured and analyzed. Activation (in terms of hemodynamic responses) as well as functional connectivity and lateralization were calculated for both language paradigms (Word Expression and Word Reception).

During the word expression paradigm, the activation results show there is relative increase in oxygen supplies both in the anterior fronto-temporal region (encompassing Broca's area and its right hemisphere analog) as well as in the posterior fronto-temporal region (encompassing Wernicke's area and its right hemisphere analog) during the verbal fluency task (Word Generation task). Yet, during this task, while there is an increase in oxygen there is very little consumption of oxygen by the brain. The reason behind this may be due to the fact that while the brain increases its oxygen supplies in anticipation of the need for oxygen, the task is simple, and thus minimal consumption of oxygen is observed.

Furthermore, results from the functional connectivity analysis show that there are strong positive correlations (left vs. right) during the verbal fluency task in both the anterior fronto- 
temporal region (encompassing Broca's area and its right hemisphere analog) and posterior fronto-temporal region (encompassing Wernicke's area and its right hemisphere analog). Results from the functional connectivity analysis also show that there is changing connectivity patterns in both the anterior fronto-temporal region, and posterior fronto-temporal region, for each of the three stimuli (Word Generation (W.G), Jaw Movement (J.M), and Rest (R)) presented during the Word Expression paradigm. This is interpreted to mean that the neuronal pathways used in the brain during a verbal fluency task (word generation task) are different than those used during a jaw movement task, or rest task. Even though the results from the functional connectivity analysis show there are strong correlations between left and right hemispheres during the verbal fluency task ( i.e. the two hemispheres for each region are synchronous with one another), the lateralization analysis shows that the left anterior region (encompassing Broca's area) becomes dominant during the verbal fluency task. Overall it is believed that during a word expression task, the left anterior region (encompassing Broca's area) is preferably more activated, and is majorly involved in the expression of language in the brain in right handed individuals.

During the word reception paradigm, the activation trend is opposite to that observed in the word expression paradigm, where there is now a decrease in the relative amount of oxygen supplies in the brain due to higher consumption of oxygen in both the anterior fronto-temporal (encompassing Broca's area and its right hemisphere analog) and posterior fronto-temporal (encompassing Wernicke's region and its right hemisphere analog) regions. This is due to the fact that the brain in these regions are trying to comprehend the language being spoken to it, and thus requires more oxygen supply in order to comprehend what is being spoken (both in the forward audio task (comprehensible speech), and in the reverse audio task (non-comprehensible 
speech). Results from the functional connectivity analysis show strong positive (left vs. right) correlations in the posterior fronto-temporal region (encompassing Wernicke's area and its right hemisphere analog), but not in the anterior fronto-temporal region (encompassing Broca's area and its right hemisphere analog). Results from the functional connectivity analysis also show that there is changing connectivity patterns in both the anterior fronto-temporal region, and posterior fronto-temporal region, for each of the three stimuli (Forward Audio (F.A), Reverse Audio (R.A), and Rest (R)) presented during the Word Reception paradigm. This is again interpreted to mean that the neuronal pathways utilized by the anterior temporal and posterior temporal regions differ during a comprehensible speech task, from both a non-comprehensible speech task, as well as a rest task. Furthermore, the neuronal pathways utilized during a word reception paradigm also differ from those used during a word expression paradigm. Lateralization results show that for both language reception tasks the right hemisphere is dominant, while during the forward audio task both the right anterior and posterior regions are dominant, but during the reverse audio task (non-comprehensible speech) only the right anterior region is dominant. Overall, during a word reception task it is believed that the right posterior fronto-temporal region (encompassing the right analog of Wernicke's area) is highly involved in language reception, with the right anterior region (encompassing the right analog of Broca's area) also playing a role in language reception in right handed individuals.

Activation analysis by itself tells of how the brain demands and consumes oxygen during a given stimuli. From this, the simplicity or complexity of tasks can be determined, and also the trends exhibited during these tasks can be observed. Functional connectivity tells about the neuronal pathways utilized during these tasks, as well as the synchrony between the language 
areas, and their right hemisphere analogs. Lateralization analysis tells of the dominance of one hemisphere over another, meaning that even though they may both be synchronous one region is playing a major role in language function, while the other is playing only a supporting role. By utilizing the activation analysis, as well as functional connectivity analysis, and lateralization analysis, a better understanding of how the brain thinks with regards to language can be uncovered. Correlation of these three aspects give information regarding not only relative change in oxygen consumption, and task complexity, but also the neuronal pathways utilized, synchrony between language regions, and dominance of one region over the other. By providing information from all three analyses, a better understanding of specific language function can be given. By performing these three analyses together under multiple language paradigms understanding of how each language region functions under different language function can also be drawn. In the future this will allow researchers to further understand the dynamic roles each region plays in language, as it pertains to different language functions as well (i.e. word expression, word reception, etc.). In the future the goal will be to apply these same two language paradigms in epileptic populations, as an ongoing effort to better understand language function in the brain, but also how language is affected when dealing with epileptic populations. This study may also be combined with another study performed by our group in the frontal region, to gain an understanding into the cognitive function, as well as the language function of the brain both in frontal and temporal regions. In the near future, this study may be used by clinical researcher's and surgeons as a method for mapping language function and cognition in the brain, both prior to and after removal of epileptic foci, both as a guide and as a tool to measure the effects of surgery. 


\section{Appendices}

\section{Appendix A}

\begin{tabular}{|c|c|c|c|c|}
\hline Title & Author(s) & $\begin{array}{c}\text { Region of } \\
\text { Interest(ROI) }\end{array}$ & Stimuli & Results \\
\hline $\begin{array}{l}\text { "Temporal dynamics of } \\
\text { perisylvian activation } \\
\text { during language } \\
\text { processing in children and } \\
\text { adults" }\end{array}$ & $\begin{array}{l}\text { Brauer, J., Neumann, J., } \\
\text { Friederici, A.D (2008) }\end{array}$ & Temporal Region & Word Reception tasks & $\begin{array}{c}\text { Overall latency } \\
\text { differences between } \\
\text { adults and children are } \\
\text { assumed to be due to } \\
\text { maturing of perisylvian } \\
\text { regions, and connections } \\
\text { between them. }\end{array}$ \\
\hline $\begin{array}{l}\text { "Temporal cortex } \\
\text { activation during speech } \\
\text { recognition: an optical } \\
\text { topography study" }\end{array}$ & $\begin{array}{l}\text { Sato, T., Takeuchi,K., } \\
\text { Sakai,L.(1999) }^{[36]}\end{array}$ & Temporal Region & $\begin{array}{l}\text { Word Reception Listening } \\
\text { tasks }\end{array}$ & $\begin{array}{c}\text { Results suggest that } \\
\text { activity in left temporal } \\
\text { associated area reflects } \\
\text { load of auditory memory } \\
\text { and language information } \\
\text { processing }\end{array}$ \\
\hline $\begin{array}{c}\text { "Hand Actions and } \\
\text { Speech Representation in } \\
\text { Broca's Area" }\end{array}$ & 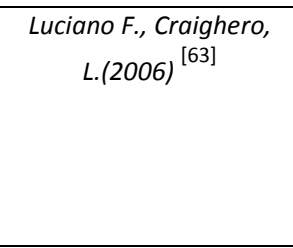 & Broca's Area & $\begin{array}{c}\text { Word Generation Lexical } \\
\text { decision tasks }\end{array}$ & $\begin{array}{l}\text { Broca's Area may be } \\
\text { involved in meaningful } \\
\text { action recognition, in } \\
\text { addition to speech } \\
\text { activation, it may also be } \\
\text { activated by meaningful } \\
\text { hand or mouth actions }\end{array}$ \\
\hline $\begin{array}{l}\text { "Cerebral blood flow } \\
\text { during object naming and } \\
\text { word reading" }\end{array}$ & $\begin{array}{l}\text { Bookheimer et. } \\
\text { al(1995) }^{[64]}\end{array}$ & $\begin{array}{c}\text { Posterior Temporal } \\
\text { Region }\end{array}$ & Word Generation Task & $\begin{array}{l}\text { Speech tasks and reading } \\
\text { tasks both produce } \\
\text { activations, silent reading } \\
\text { tasks have same } \\
\text { activation, different } \\
\text { network. }\end{array}$ \\
\hline $\begin{array}{l}\text { "The anatomy of } \\
\text { phonological and } \\
\text { semantic processing in } \\
\text { normal subjects" }\end{array}$ & Demonet e. al(1992) ${ }^{[43]}$ & $\begin{array}{l}\text { Broca's area and } \\
\text { Wernicke's area }\end{array}$ & $\begin{array}{l}\text { Word Generation tasks, } \\
\text { Word Reception tasks }\end{array}$ & $\begin{array}{l}\text { No difference found } \\
\text { between broca's area and } \\
\text { Left Superior Temporal } \\
\text { Gyrus, suggests these } \\
\text { areas activated by } \\
\text { phonological component } \\
\text { of both tasks. }\end{array}$ \\
\hline $\begin{array}{l}\text { "The age of second } \\
\text { language acquisition } \\
\text { determines the variability } \\
\text { in activation elicited by } \\
\text { narration in three } \\
\text { languages in Broca's and } \\
\text { Wernicke's area" }\end{array}$ & Bloch et. al(2009) ${ }^{[44]}$ & $\begin{array}{l}\text { Broca's area and } \\
\text { Wernicke's area }\end{array}$ & Word Reception tasks & $\begin{array}{l}\text { Bilateral activation in } \\
\text { wernickes area, } \\
\text { Simultaneous bilinguals } \\
\text { show overlap in the } \\
\text { activation for all three } \\
\text { languages and bilateral } \\
\text { activation in wernickes } \\
\text { area. Overall regional } \\
\text { activation patterns show } \\
\text { no major difference } \\
\text { between age groups and } \\
\text { languages }\end{array}$ \\
\hline
\end{tabular}




\begin{tabular}{|c|c|c|c|c|}
\hline $\begin{array}{l}\text { "Differential effect of side } \\
\text { of temporal lobe epilepsy } \\
\text { on lateralization of } \\
\text { hippocampal, } \\
\text { tempolateral, and inferior } \\
\text { frontal activation } \\
\text { patterns during a verbal } \\
\text { episodic memory task" }\end{array}$ & Wagner et. al (2008) ${ }^{[45]}$ & $\begin{array}{l}\text { Fronto-temporal, and } \\
\text { temporal region( } \\
\text { encompassing both } \\
\text { Broca's area and } \\
\text { Wernicke's area }\end{array}$ & $\begin{array}{l}\text { Combination of Word } \\
\text { generation and Word } \\
\text { reception tasks }\end{array}$ & $\begin{array}{l}\text { Study demonstrates } \\
\text { patients w/LTLE } \\
\text { activation patterns during } \\
\text { verbal episodic memory } \\
\text { task within MTL are less } \\
\text { left lateralized than in } \\
\text { patients with RTLE, } \\
\text { further analysis showed } \\
\text { significant difference } \\
\text { between laterality in } \\
\text { medial temporal vs. } \\
\text { frontal activation in } \\
\text { patients with LTLE }\end{array}$ \\
\hline $\begin{array}{l}\text { "Beyond Superior } \\
\text { Temporal Cortex: Inter- } \\
\text { subject Correlations in } \\
\text { Narrative Speech } \\
\text { Comprehension" }\end{array}$ & Wilson et. al (2008) & Bilateral temporal region & Word Reception tasks & $\begin{array}{l}\text { For auditory narrative } \\
\text { only, bilateral activation } \\
\text { of temporal region was } \\
\text { seen, as well there were } \\
\text { high correlations } \\
\text { between left and right } \\
\text { temporal regions, } \\
\text { propose network of } \\
\text { regions beyond superior } \\
\text { temporal cortex } \\
\text { important for higher level } \\
\text { linguistic processes }\end{array}$ \\
\hline
\end{tabular}

Table A1. A list of various research performed by other groups pertaining to language activation in the brain using both NIRS as

well as various other modalities.

\begin{tabular}{|c|c|c|c|c|}
\hline Title & ROI & Stimuli & Modality & Result/Conclusion \\
\hline $\begin{array}{l}\text { "Aberrant } \\
\text { Functional } \\
\text { Connectivity in } \\
\text { Autism: Evidence } \\
\text { from low frequency } \\
\text { BOLD signal } \\
\text { fluctuations }{ }^{[65]}\end{array}$ & $\begin{array}{l}\text { Left middle frontal } \\
\text { gyrus, left superior } \\
\text { parietal cortex, left } \\
\text { middle occipital } \\
\text { cortex }\end{array}$ & $\begin{array}{l}\text { Word generation } \\
\text { task consisting of } \\
\text { identifying words } \\
\text { that could be } \\
\text { touched, (did by } \\
\text { visual, and auditory } \\
\text { presented) }\end{array}$ & fMRI & $\begin{array}{l}\text { Not applicable to } \\
\text { your work }\end{array}$ \\
\hline $\begin{array}{l}\text { "Brain dynamics } \\
\text { during natural } \\
\text { viewing conditions- } \\
\text { A new guide for } \\
\text { mapping } \\
\text { connectivity in vivo" } \\
\text { A. Bartels S. Zeki } \\
\text { [48] }\end{array}$ & $\begin{array}{l}\text { "Visual, auditory, } \\
\text { language areas }\end{array}$ & $\begin{array}{l}\text { Watched and } \\
\text { listened to movie, } \\
\text { with } 830 \mathrm{~s} \text { rest } \\
\text { periods in the } \\
\text { movie (overall time } \\
22 \mathrm{~min} 25 \mathrm{~s})\end{array}$ & fMRI & $\begin{array}{l}\text { Found in language } \\
\text { areas, that } \\
\text { anatomically } \\
\text { connected regions( } \\
\text { Broca, Wernicke) } \\
\text { show connectivity } \\
\text { during rest, but } \\
\text { higher during natural } \\
\text { viewing state }\end{array}$ \\
\hline $\begin{array}{l}\text { "Detection of } \\
\text { functional } \\
\text { connectivity using }\end{array}$ & $\begin{array}{l}\text { Broca's area, } \\
\text { Wernicke's area, } \\
\text { prefrontal cortex }\end{array}$ & $\begin{array}{l}\text { Listened to story } \\
\text { task,as well as rest } \\
\text { period, }\end{array}$ & fMRI & $\begin{array}{l}\text { Correlations } \\
\text { between } \\
\text { broca/wernicke were } \\
\text { significant during }\end{array}$ \\
\hline
\end{tabular}




\begin{tabular}{|c|c|c|c|c|}
\hline $\begin{array}{l}\text { temporal } \\
\text { correlations in MR } \\
\text { images" Hampson } \\
2002^{[47]}\end{array}$ & & & & $\begin{array}{l}\text { both rest and speech, } \\
\text { but correlations } \\
\text { during speech were } \\
\text { significantly greater, }\end{array}$ \\
\hline $\begin{array}{l}\text { "Detecting resting } \\
\text { state functional } \\
\text { connectivity in the } \\
\text { language system } \\
\text { using functional near } \\
\text { infrared } \\
\text { spectroscopy", } \\
\text { Zhang et. al }{ }^{[35]}\end{array}$ & $\begin{array}{l}\text { Bilateral frontal and } \\
\text { temporal regions }\end{array}$ & $\begin{array}{l}\text { Generate verbs after } \\
\text { hearing concrete } \\
\text { nouns(VFT), and a } \\
\text { resting state } \\
\text { functional } \\
\text { connectivity analysis } \\
\text { as well(RSFC) }\end{array}$ & fNIRS & $\begin{array}{l}\text { Results show } \\
\text { connectivity is } \\
\text { similar in VFT and } \\
\text { rest. Left hemisphere } \\
\text { dominance during } \\
\text { language RSFC, and } \\
\text { RSFC, language } \\
\text { tasks show higher } \\
\text { left hemisphere } \\
\text { dominance }\end{array}$ \\
\hline $\begin{array}{l}\text { Comparative } \\
\text { cytoarchitectonic } \\
\text { analysis of the } \\
\text { human and the } \\
\text { macque ventrolateral } \\
\text { prefrontal cortex and } \\
\text { corticocortical } \\
\text { connection patterns } \\
\text { in the monkey". } \\
\text { Petrides, M., } \\
\text { Pandya, D.N }\end{array}$ & $\begin{array}{l}\text { Ventrolateral } \\
\text { prefrontal cortex }\end{array}$ & $\begin{array}{l}\text { Looked at } \\
\text { cytoarchitechture of } \\
\text { both human brain in } \\
\text { Broadmann areas } \\
\text { 45(Broca), as well as } \\
\text { BA 25( Wernicke), } \\
\text { compared } \\
\text { connectivity of } \\
\text { cytoarchitecture both } \\
\text { in Macaque and } \\
\text { Human }\end{array}$ & $\begin{array}{l}\text { Architectonic } \\
\text { analysis }\end{array}$ & $\begin{array}{l}\text { Found BA45 } \\
\text { showed stronger } \\
\text { clusters of brain cell } \\
\text { activity showing a } \\
\text { stronger connectivity } \\
\text { in superior temporal } \\
\text { gyrus of human } \\
\text { brain. Area } \\
\text { 47/12(BA) show } \\
\text { connectivity to } \\
\text { rostral } \\
\text { inferotemporal } \\
\text { region, while BA 45 } \\
\text { shows stronger } \\
\text { connectivity to } \\
\text { superior temporal } \\
\text { gyrus, Furthermore } \\
\text { this region shows } \\
\text { activity during } \\
\text { delayed memory } \\
\text { tasks using active } \\
\text { judgement((e.g. } \\
\text { Courtney et al., } \\
\text { 1996; } \\
\text { Petrides et al. 2002), } \\
\text { and on tasks } \\
\text { requiring active } \\
\text { retrieval processes } \\
\text { via PET(Cadoret et } \\
\text { al. 2001; } \\
\text { Kostopoulos \& } \\
\text { Petrides, 2001) }\end{array}$ \\
\hline $\begin{array}{l}\text { "The } \\
\text { neuroanatomical and } \\
\text { functional } \\
\text { organization of } \\
\text { speech perception" }\end{array}$ & $\begin{array}{l}\text { Looked into } \\
\text { different theories of } \\
\text { speech perception, } \\
\text { as well reviewed } \\
\text { past literature to give } \\
\text { insight into the fact }\end{array}$ & & & \\
\hline
\end{tabular}




\begin{tabular}{|c|c|c|c|c|}
\hline $\begin{array}{l}\text { Scott, S.K., } \\
\text { Johnsrude, I.S }\end{array}$ & $\begin{array}{l}\text { that speech may } \\
\text { activate different } \\
\text { portions of the brain } \\
\text { depending upon the } \\
\text { type of speech } \\
\text { perceived, i.e a } \\
\text { "where", or a "what" } \\
\text { speech, furthermore } \\
\text { Wernicke's area } \\
\text { encompasses a large } \\
\text { portion of the } \\
\text { posterior temporal } \\
\text { region, which may } \\
\text { mean that it } \\
\text { encompasses both } \\
\text { speech recognition } \\
\text { systems, as well as } \\
\text { speech output, and } \\
\text { that Broca's area be } \\
\text { involved in some } \\
\text { speech processing } \\
\text { tasks, including } \\
\text { explicit speech } \\
\text { sound segmentations }\end{array}$ & & & \\
\hline $\begin{array}{l}\text { "Functional Brain } \\
\text { mapping detected by } \\
\text { cortical stimulations } \\
\text { using chronically } \\
\text { implanted } \\
\text { electrodes" } \\
\text { Hoshida, T., } \\
\text { Hirabayashi, H., } \\
\text { Kaido, T. }{ }^{[49]}\end{array}$ & $\begin{array}{l}\text { Anterior language } \\
\text { areas( not restricted } \\
\text { to just BA } 44 / 45 \text { i.e } \\
\text { Broca's area), } \\
\text { Posterior language } \\
\text { areas were also } \\
\text { investigated but not } \\
\text { restricted to the so } \\
\text { called Wernicke's } \\
\text { area }\end{array}$ & $\begin{array}{l}\text { Used spontaneous } \\
\text { speech, picture } \\
\text { naming, Auditory } \\
\text { comprehension, and } \\
\text { Responsive naming } \\
\text { tasks }\end{array}$ & EEG & $\begin{array}{l}\text { Can recognize and } \\
\text { differentiate definite } \\
\text { and non definite or } \\
\text { core to peripheral } \\
\text { language areas by } \\
\text { performing various } \\
\text { language tasks, } \\
\text { Comprehension } \\
\text { tasks are necessary } \\
\text { even in the } \\
\text { identification of } \\
\text { Broca's area, } \\
\text { Responsive naming } \\
\text { tasks are more } \\
\text { effective in } \\
\text { differentiating } \\
\text { anterior and } \\
\text { posterior language } \\
\text { areas }\end{array}$ \\
\hline $\begin{array}{l}\text { "Functional } \\
\text { Connectivity and } \\
\text { neurological } \\
\text { recovery" } \\
\text { Corbetta, M. }{ }^{[68]}\end{array}$ & $\begin{array}{l}\text { Review paper } \\
\text { highlighting the } \\
\text { various aspects of } \\
\text { brain functional } \\
\text { connectivity( } \\
\text { defining, and } \\
\text { explaining it as not } \\
\text { just static noise, but } \\
\text { actual anatomical } \\
\text { fluctuations of two } \\
\text { homologous }\end{array}$ & & & \\
\hline
\end{tabular}




\begin{tabular}{|c|c|c|c|c|}
\hline & $\begin{array}{l}\text { hemispheres during } \\
\text { a given stimuli, or } \\
\text { even during rest), }\end{array}$ & & & \\
\hline $\begin{array}{l}\text { "Functional MR } \\
\text { imaging exposes } \\
\text { differential brain } \\
\text { responses to syntax } \\
\text { prosody during } \\
\text { auditory sentence } \\
\text { comprehension" } \\
\text { Meyer, M., Alter, } \\
\text { K., Freiderici, A. }{ }^{[69]}\end{array}$ & $\begin{array}{l}\text { Superior temporal } \\
\text { region, anterior } \\
\text { superior temporal } \\
\text { region(planum } \\
\text { polare), fronto- } \\
\text { opercular region } \\
\text { bilaterally }\end{array}$ & $\begin{array}{l}\text { Listened to } \\
\text { sentences either } \\
\text { focused on lexical, } \\
\text { synactic, or prosodic } \\
\text { information }\end{array}$ & fMRI & $\begin{array}{l}\text { Statistical analysis } \\
\text { found different } \\
\text { activation patterns } \\
\text { for frontal and } \\
\text { temporal regions of } \\
\text { brain,degraded } \\
\text { speech produced } \\
\text { stronger activation in } \\
\text { frontal region, for } \\
\text { STR, inverse pattern } \\
\text { was found that } \\
\text { showed higher } \\
\text { activation in anterior } \\
\text {, mid, and posterior } \\
\text { of left supratemporal } \\
\text { plane, particular in } \\
\text { the planum polare, } \\
\text { and left planum } \\
\text { temporal mediate } \\
\text { syntactic and } \\
\text { semantic processing, } \\
\text { whereas right fronto- } \\
\text { lateral areas seem to } \\
\text { be more sensitive to } \\
\text { prosodic cues } \\
\text { available in spoken } \\
\text { language }\end{array}$ \\
\hline $\begin{array}{l}\text { "Use of fNIRS to } \\
\text { assess resting state } \\
\text { functional } \\
\text { connectivity" } \\
\text { Lu, C.M, Zhang, Y- } \\
\text { J.,Biswal, B. Zang, } \\
\text { YF., Peng, DL.,Zhu, } \\
\text { CZ., }{ }^{\text {[35] }}\end{array}$ & $\begin{array}{l}\text { Sensorimotor and } \\
\text { auditory cortex }\end{array}$ & $\begin{array}{l}\text { Resting state } \\
\text { conditions }\end{array}$ & FNIRS & $\begin{array}{l}\text { Found seed based } \\
\text { analysis comparable } \\
\text { to fMRI findings, } \\
\text { validated that fNIRS } \\
\text { is suitable to } \\
\text { perform resting state } \\
\text { functional } \\
\text { connectivity analysis }\end{array}$ \\
\hline $\begin{array}{l}\text { "Lateralization of } \\
\text { receptive language } \\
\text { function using near } \\
\text { infrared } \\
\text { spectroscopy" } \\
\text { Paquette, N. }{ }^{[62]}\end{array}$ & $\begin{array}{l}\text { Covered both left } \\
\text { and right } \\
\text { homologous regions } \\
\text { corresponding to the } \\
\text { areas known as } \\
\text { Broca's region and } \\
\text { Wernicke's region }\end{array}$ & $\begin{array}{l}\text { Used two stories that } \\
\text { were presented to } \\
\text { subjects( a known } \\
\text { story, and an } \\
\text { unknown story) in } \\
\text { two languages( a } \\
\text { known language( } \\
\text { French) and an } \\
\text { unknown language( } \\
\text { Arabic)) }\end{array}$ & fNIRS & $\begin{array}{l}\text { Findings suggest } \\
\text { that there is a higher } \\
\text { activation pattern } \\
\text { during French story } \\
\text { suggesting left } \\
\text { hemisphere } \\
\text { dominance for } \\
\text { receptive language, } \\
\text { Arabic stories } \\
\text { induced no specific } \\
\text { patterns, consistent } \\
\text { with previous pet } \\
\text { and fMRI findings, } \\
\text { left temporal gyrus, } \\
\text { and left middle }\end{array}$ \\
\hline
\end{tabular}




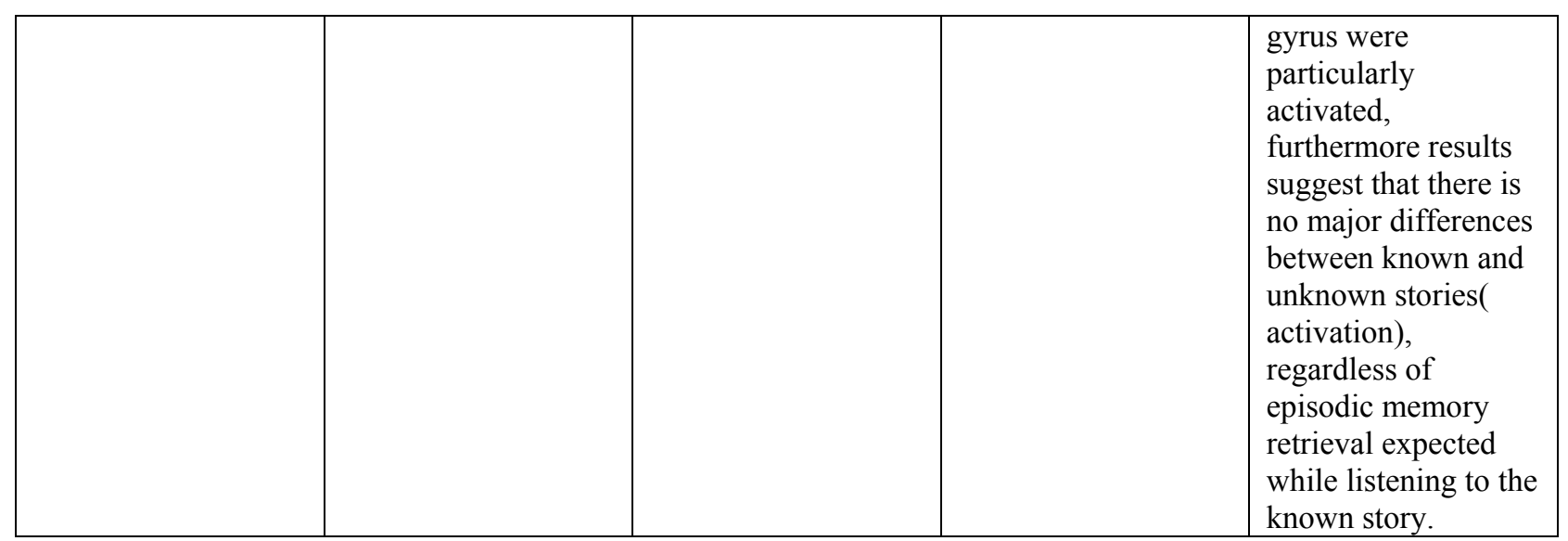

Table A2. A list of various research perfomed by other groups pertaining to functional connectivity and lateralization in the brain using both NIRS as well as various other modalities.

\section{Appendix B}

In order to post process the data acquired from each subject, certain data analysis techniques were used in order to filter, prune, average, and then calculate the relative concentration change for each hemodynamic response function. These steps were all performed using the statistical software HomER. HomER offers the ability to filter the raw signal, prune the measurements, and then average the data. As mentioned previously, the data was filtered due to the fact that there are noise artifacts introduced into the signal due to physiological fluctuations of the body. Once the signal is filtered it is then pruned. Since the hemodynamic response to a stimuli occurs roughly 10 seconds after the stimuli was presented, the data is pruned in order to have the start of the activation in response to a stimuli at time equal to zero. Once the pruning step has taken place, the data is then averaged from the entire 5 block paradigm into a single block, consisting of the average for each stimuli across the 5 blocks. These averages are created for $\mathrm{HbO}, \mathrm{HbR}$, and $\mathrm{HbT}$, once individual averages have been created, the data is then taken out of HomER and exported to MATLAB which is used to create the grand average across all 
subjects. For brevity, only channel L8 will be shown, and only data for $\mathrm{HbO}$ relative concentrations are shown. These figures are implemented to illustrate the steps taken using HomER to process the raw optical signals for each paradigm for all subject replicates. The steps taken are again applied to get information regarding $\mathrm{HbR}$, as well as $\mathrm{HbT}$.

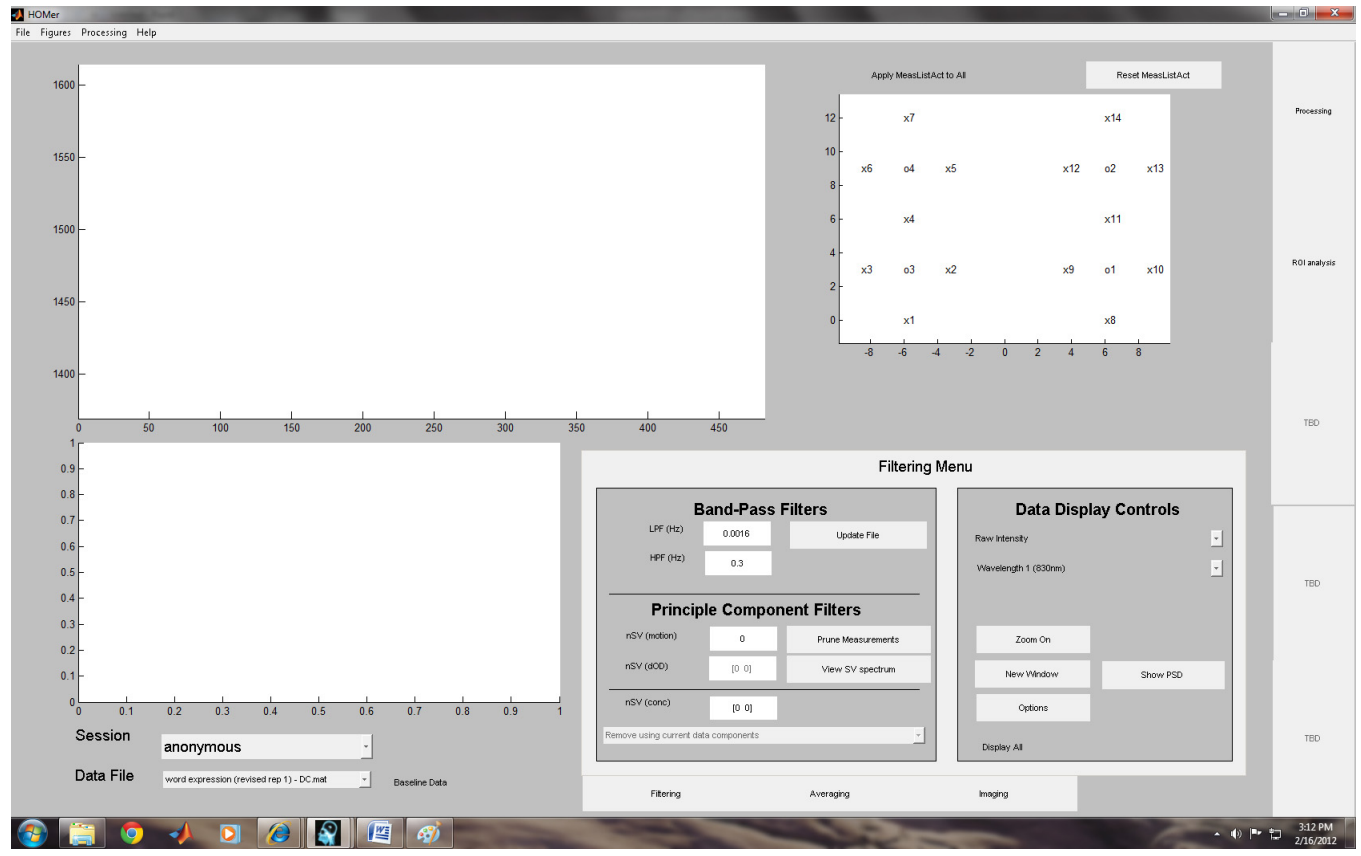

Figure B1. Figure showing HomER GUI interface before any data has been imported to the GUI. 


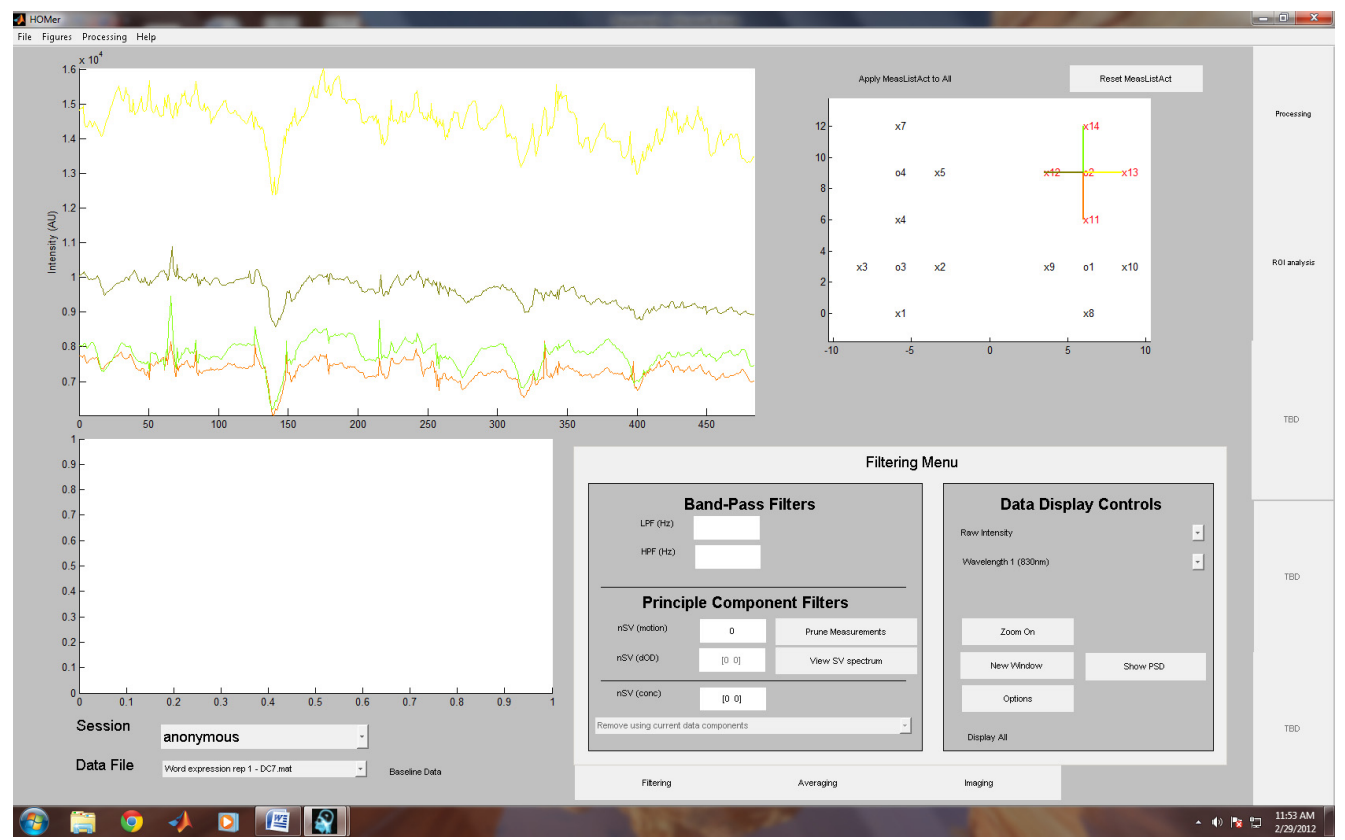

Figure B2. Figure showing HomER GUI interface with raw optical signal before filtering for right anterior temporal region.

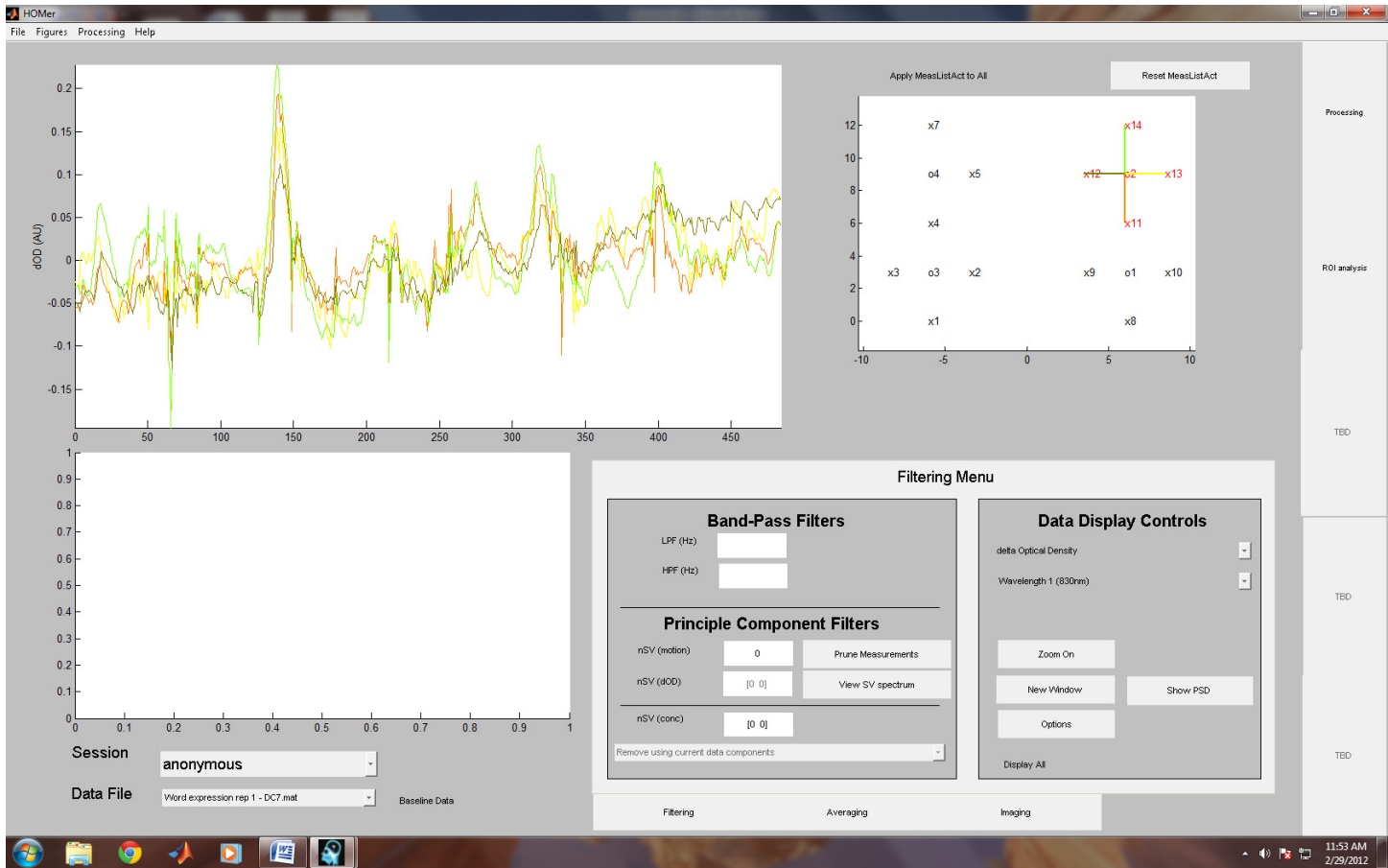

Figure B3. Figure showing HomER GUI interface with dOD signal before filtering for right anterior temporal region. 


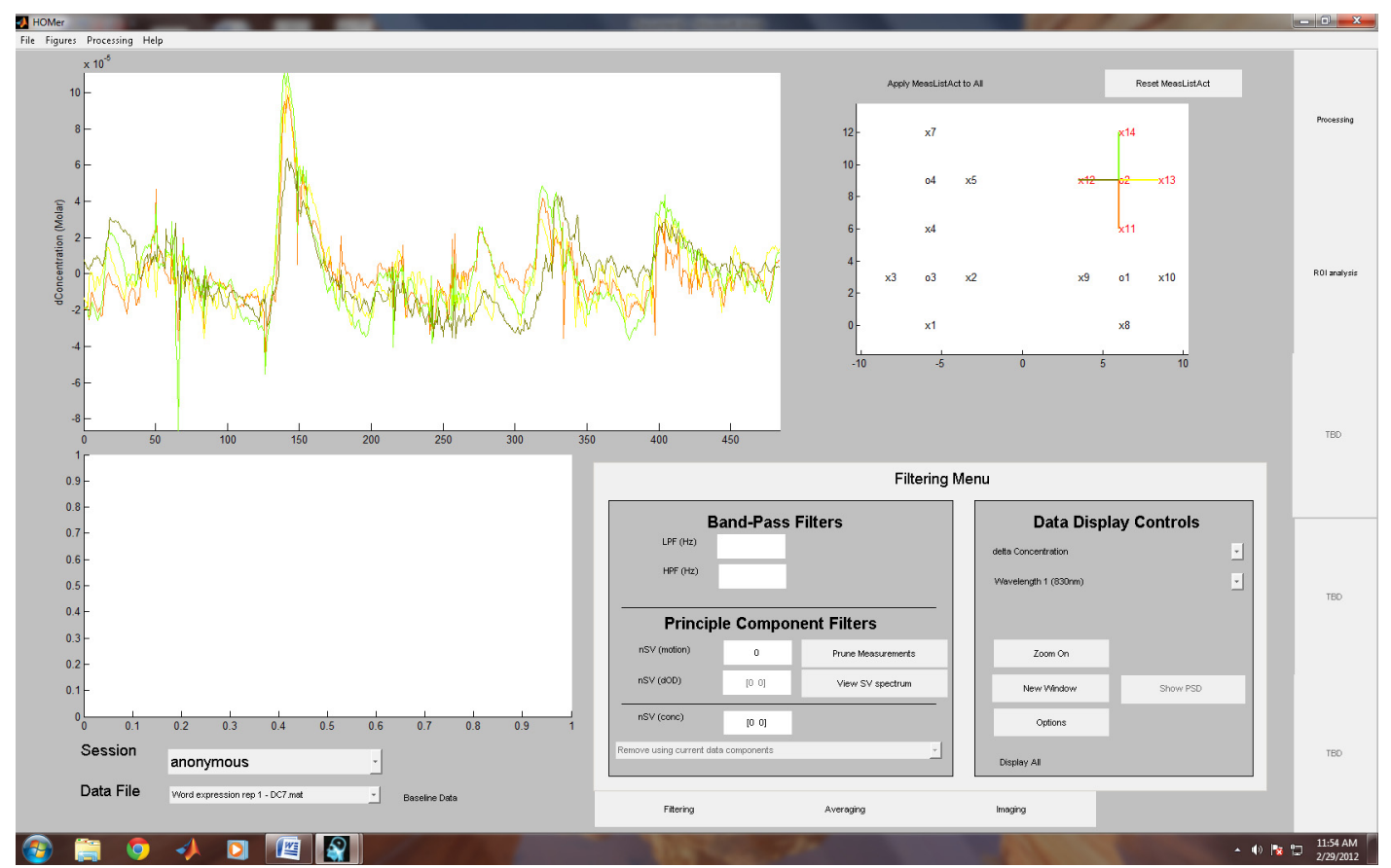

Figure B4. Figure showing HomER GUI interface with dConc of $\mathrm{HbO}$ signal before filtering for right anterior temporal region.

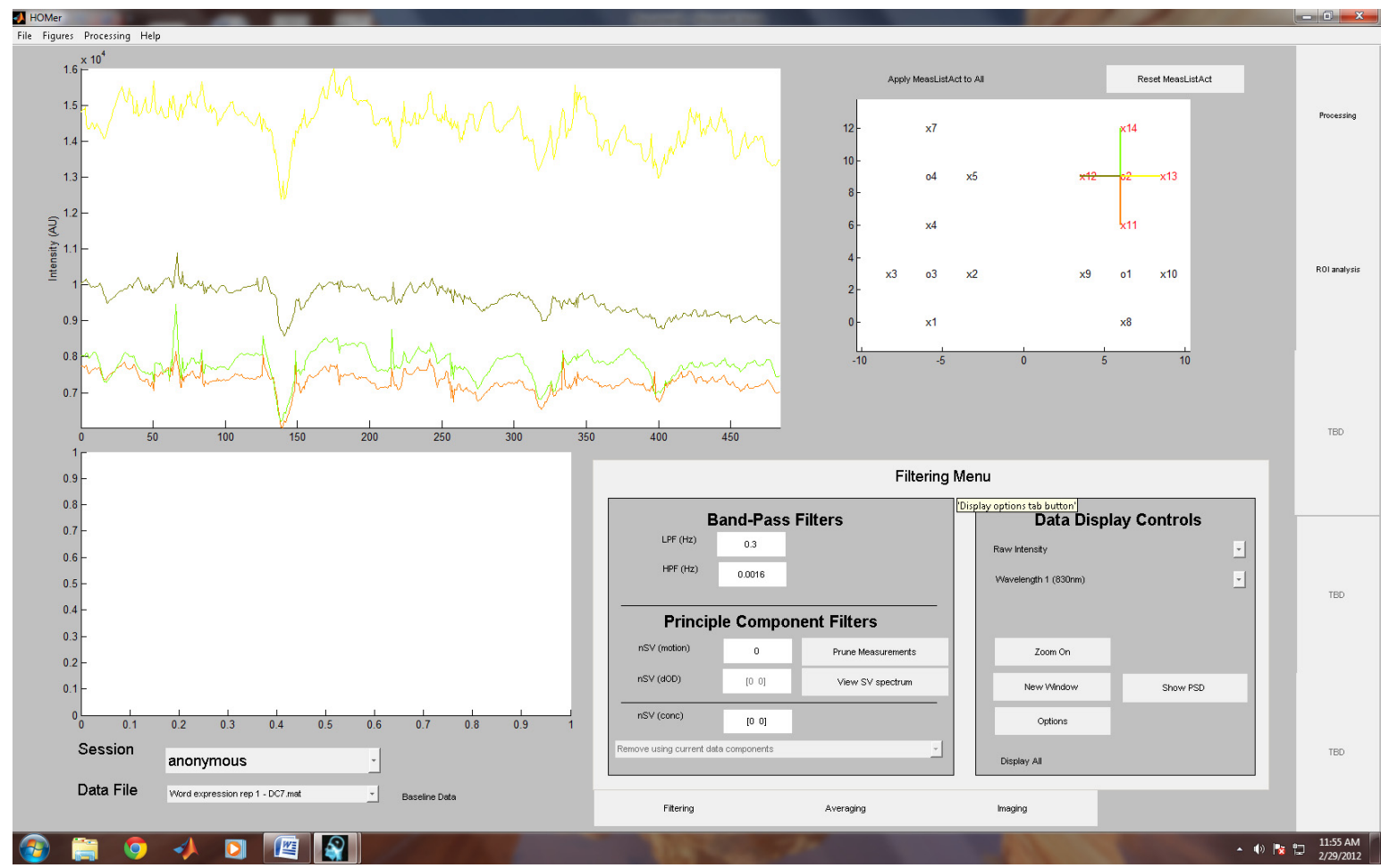

Figure B5. Figure showing HomER GUI interface with raw intensity signal after $3^{\text {rd }}$ order bandpass filtration for right anterior temporal region. 


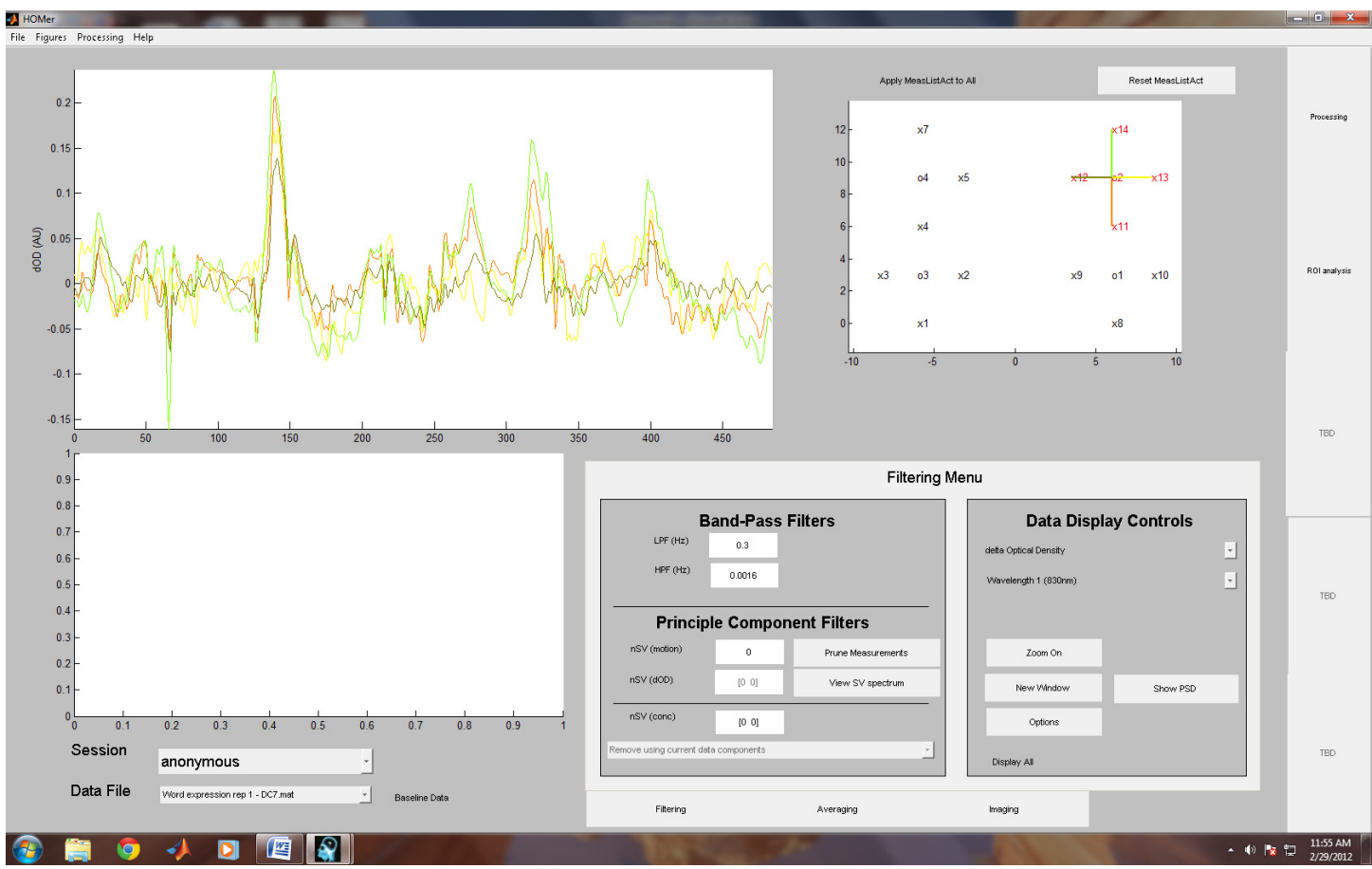

Figure B6. Figure showing HomER GUI interface with dOD signal after $3^{\text {rd }}$ order bandpass filtration for right anterior temporal region.

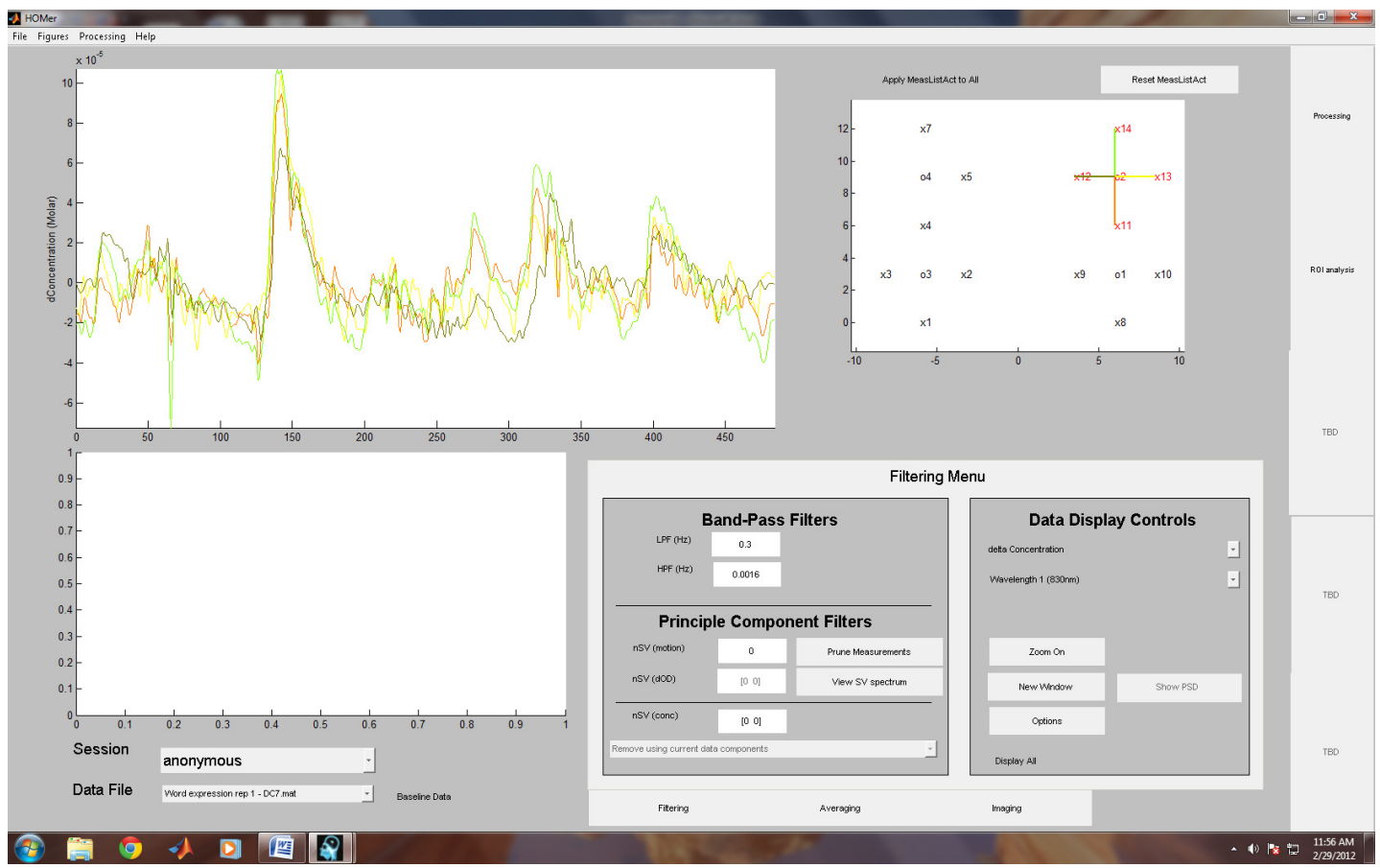

Figure B7. Figure showing HomER GUI interface with dOD signal after $3^{\text {rd }}$ order bandpass filtration for right anterior temporal region. 


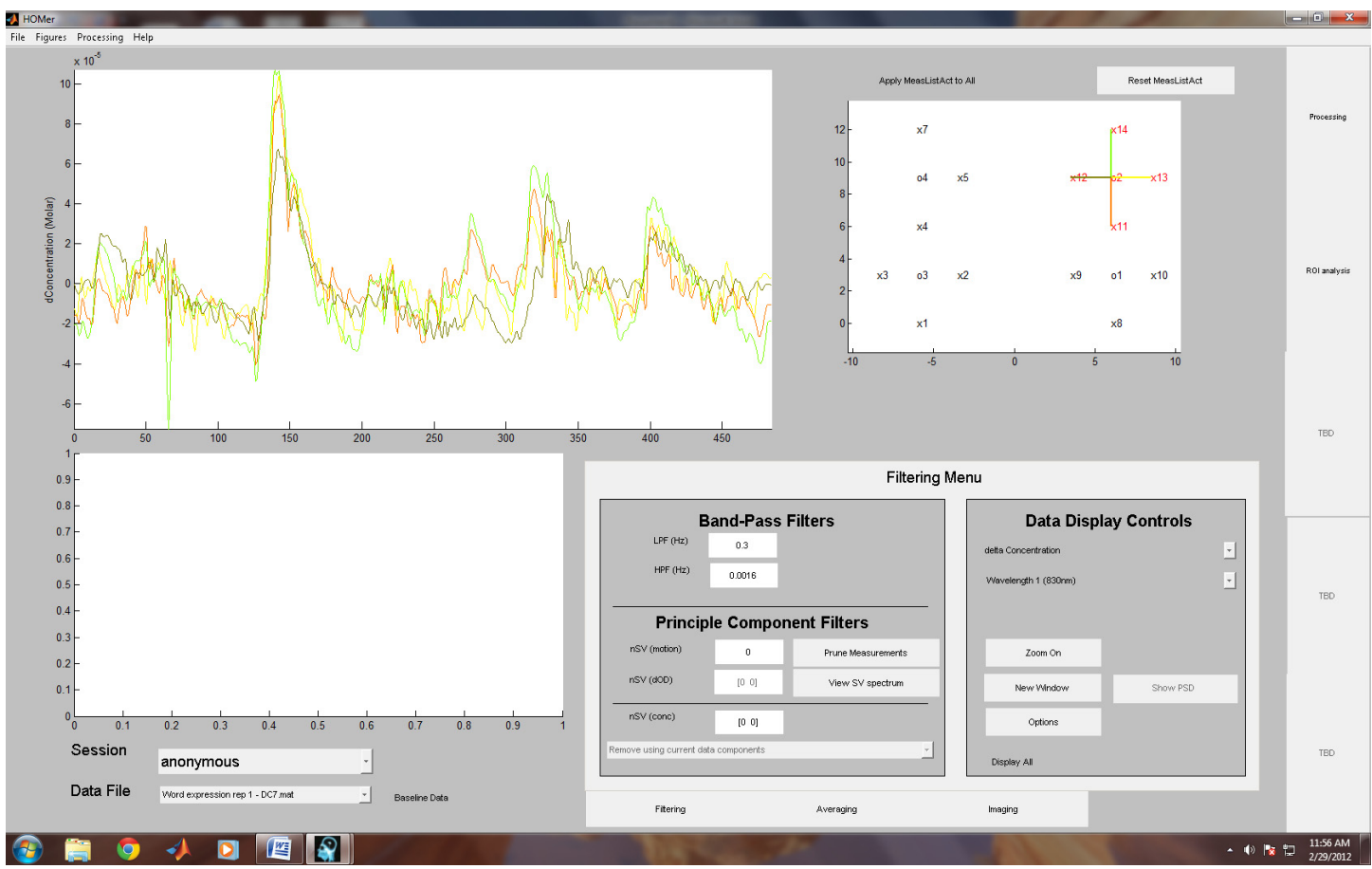

Figure B8. Figure showing HomER GUI interface with dConc of $\mathrm{HbO}$ signal after $3^{\text {rd }}$ order bandpass filtration for right anterior temporal region.

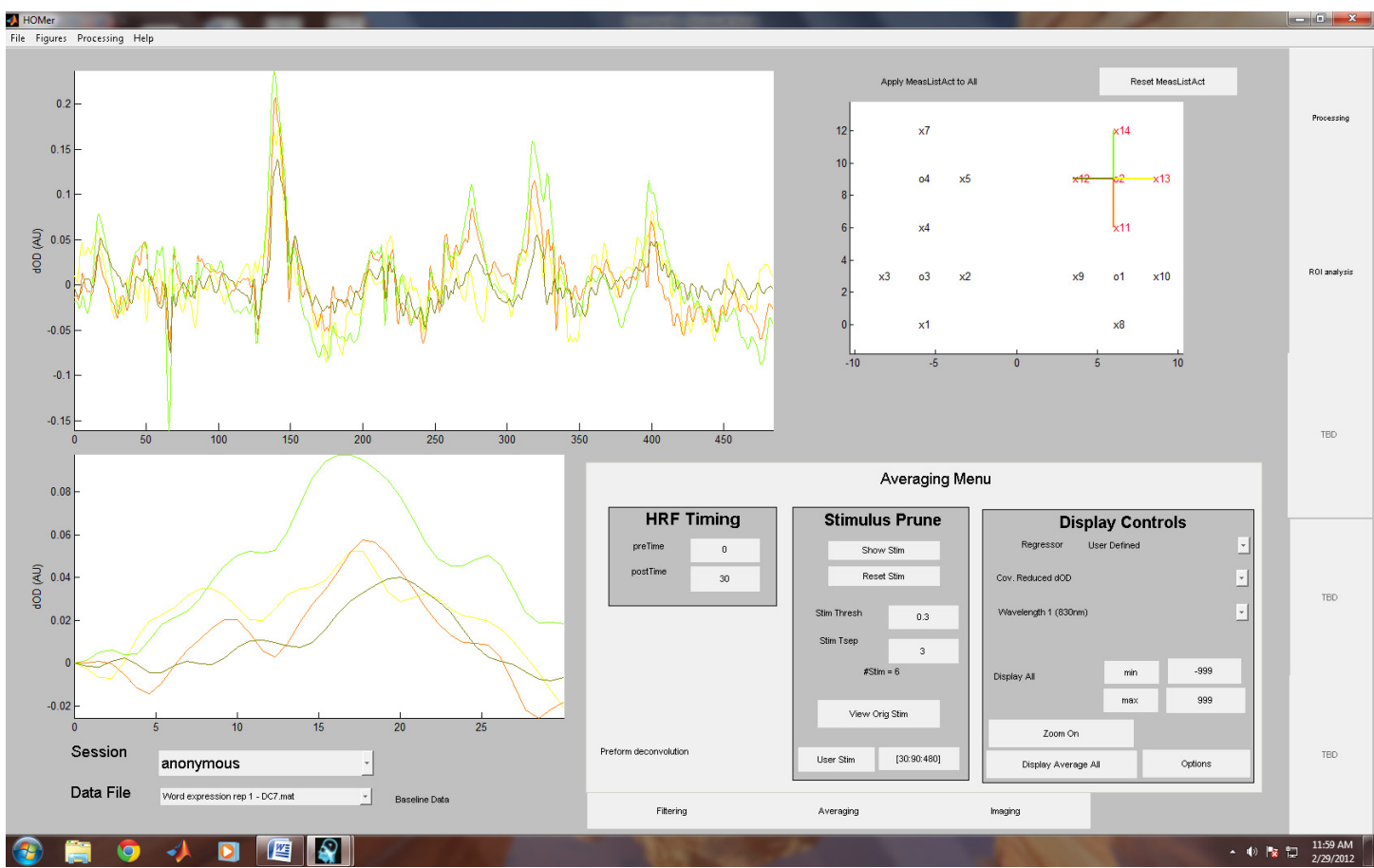

Figure B9. Figure showing HomER GUI interface with dOD of $\mathrm{HbO}$ signal after $3^{\text {rd }}$ order bandpass filtration, and averaging across 5 blocks to create a single block consisting of three thirty second stimuli for right anterior temporal region. 


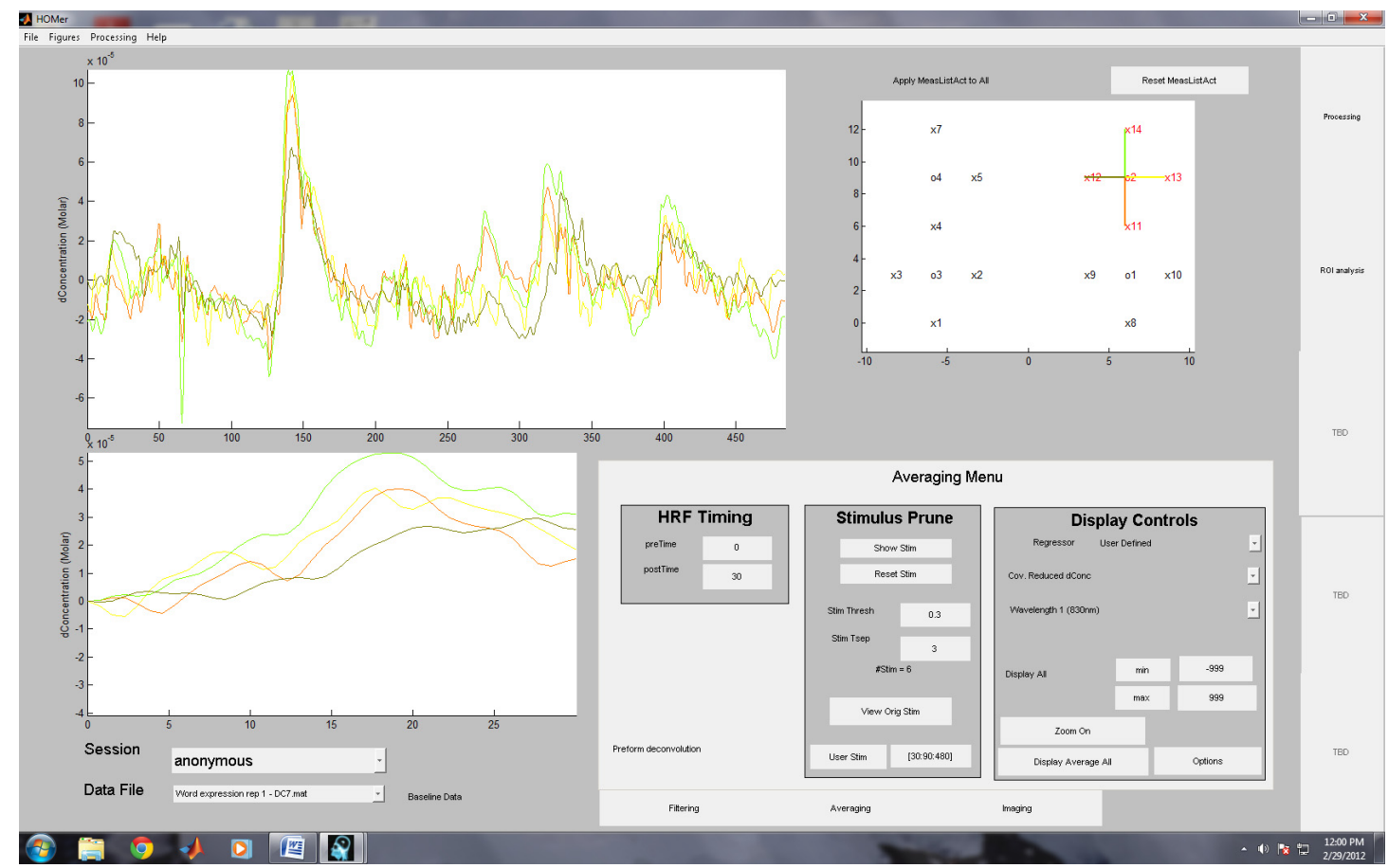

Figure B10. Figure showing HomER GUI interface with dOD of $\mathrm{HbO}$ signal after $3^{\text {rd }}$ order bandpass filtration, and averaging across 5 blocks to create a single block consisting of three thirty second stimuli for right anterior temporal region.

Appendix C- Consistency between 15 second and 30 second average

In order to test whether or not the 30-second stimulus (across each 30-sec stimuli of a given paradigm) reflects the hemodynamic response function, the 30 second HRF data was compared for consistency to the mid 15-second window of the HRF data. For this study, 2 channels were randomly selected, one channel in the right hemisphere and one channel in the left hemisphere. The 15 second average was taken from $5^{\text {th }}$ second to $20^{\text {th }}$ second for a given 30 -sec stimulus and paradigm (word expression or word reception). At each time series, within the 15 -sec and 30 -sec stimulus windows, the $\mathrm{HRF}$ data (i.e. $\mathrm{HbO}, \mathrm{HbR}$, or $\mathrm{HbT}$ ) was averaged across the blocks and trials for a given stimulus in a given paradigm. The student t-test was performed between the two averaged time-series that corresponded to the 30-sec and 15-sec window of the given 
stimulus (for a given paradigm). If the p-values are less than 0.05 , the two time series are significantly different. In the current studies performed across all stimulus in each paradigm, demonstrated that the p-values were greater than 0.05 in all cases. This implies that the 30 -sec stimulus window is consistent with the mid 15-seconds window of the same stimulus in all cases.. Tables C1-C6 show the p-value results for all the 3 stimuli in both the word expression and word reception paradigm, in terms of $\mathrm{HbO}, \mathrm{HbR}$, and $\mathrm{HbT}$.

\begin{tabular}{|c|r|r|r|}
\hline \multicolumn{4}{|c|}{ Word Expression Paradigm:15 second average vs. 30 second average (HbO) } \\
\hline Channel & Word Generation & Jaw Movement & Rest \\
\hline L2 & 0.1191 & 0.5367 & 0.4625 \\
\hline R7 & 0.8301 & 0.2038 & 0.2420 \\
\hline
\end{tabular}

Table C1. A table showing the results of the t-test in terms of p-values for the Word Expression Paradigm when comparing the 15 second average vs. the 30 second average for 2 randomly selected channels in the left and right hemispheres for $\mathrm{HbO}$, across all three stimuli. The 15 second average was selected from time 5 second through time 20 second. P values greater than 0.05 should not be considered statistically different.

\begin{tabular}{|c|r|r|r|}
\hline \multicolumn{5}{|c|}{ Word Expression Paradigm:15 second average vs. 30 second average (HbR) } \\
\hline Channel & Word Generation & Jaw Movement & Rest \\
\hline L6 & 0.1540 & 0.3766 & 0.6341 \\
\hline R3 & 0.8654 & 0.2207 & 0.9412 \\
\hline
\end{tabular}

Table C2. A table showing the results of the t-test in terms of p-values for the Word Expression Paradigm when comparing the 15 second average vs. the 30 second average for 2 randomly selected channels in the left and right hemispheres for HbR, across all three stimuli. The 15 second average was selected from time 5 second through time 20 second. $\mathrm{P}$ values greater than 0.05 should not be considered statistically different.

\begin{tabular}{|c|r|r|r|}
\hline \multicolumn{4}{|c|}{ Word Expression Paradigm:15 second average vs. 30 second average (HbT) } \\
\hline Channel & Word Generation & Jaw Movement & Rest \\
\hline L1 & 0.2800 & 0.3133 & 0.7428 \\
\hline R8 & 0.1576 & 0.4576 & 0.4074 \\
\hline
\end{tabular}

Table C3. A table showing the results of the t-test in terms of p-values for the Word Expression Paradigm when comparing the 15 second average vs. the 30 second average for 2 randomly selected channels in the left and right hemispheres for HbT, across all three stimuli. The 15 second average was selected from time 5 second through time 20 second. P values greater than 0.05 should not be considered statistically different.

\begin{tabular}{|c|r|r|r|}
\hline \multicolumn{4}{|c|}{ Word Reception Paradigm:15 second average vs. 30 second average (HbO) } \\
\hline Channel & Word Generation & Jaw Movement & Rest \\
\hline L2 & 0.4432 & 0.6233 & 0.7479 \\
\hline R7 & 0.1218 & 0.8129 & 0.2044 \\
\hline
\end{tabular}

Table C4. A table showing the results of the t-test in terms of p-values for the Word Reception Paradigm when comparing the 15 second average vs. the 30 second average for 2 randomly selected channels in the left and right hemispheres for $\mathrm{HbO}$, across all three stimuli. The 15 second average was selected from time 5 second through time 20 second. $\mathrm{P}$ values greater than 0.05 should not be considered statistically different. 


\begin{tabular}{|c|r|r|r|}
\hline \multicolumn{4}{|c|}{ Word Reception Paradigm:15 second average vs. 30 second average (HbR) } \\
\hline Channel & Word Generation & Jaw Movement & Rest \\
\hline L6 & 0.0781 & 0.3656 & 0.3982 \\
\hline R3 & 0.1220 & 0.7151 & 0.5046 \\
\hline
\end{tabular}

Table C5. A table showing the results of the t-test in terms of p-values for the Word Reception Paradigm when comparing the 15 second average vs. the 30 second average for 2 randomly selected channels in the left and right hemispheres for HbR, across all three stimuli. The 15 second average was selected from time 5 second through time 20 second. $\mathrm{P}$ values greater than 0.05 should not be considered statistically different.

\begin{tabular}{|c|r|r|r|}
\hline \multicolumn{4}{|c|}{ Word Reception Paradigm:15 second average vs. 30 second average (HbT) } \\
\hline Channel & Word Generation & Jaw Movement & Rest \\
\hline L1 & 0.2352 & 0.9745 & 0.1114 \\
\hline R8 & 0.7328 & 0.2701 & 0.4778 \\
\hline
\end{tabular}

Table C6. A table showing the results of the t-test in terms of p-values for the Word Reception Paradigm when comparing the 15 second average vs. the 30 second average for 2 randomly selected channels in the left and right hemispheres for $\mathrm{HbT}$, across all three stimuli. The 15 second average was selected from time 5 second through time 20 second. $\mathrm{P}$ values greater than 0.05 should not be considered statistically different.

\section{Appendix D- Regional Analysis Statistical Results}

For each of the four fronto-temporal regions ( Left Anterior, Right Anterior, Left Posterior, Right Posterior) that were monitored during both paradigms, there were four channels ( source-detector pair) corresponding to each region. These four separate regions encompassed the two language areas (Left anterior region encompassing Broca's area, Left posterior region encompassing Wernicke's area) as well as their right hemisphere analogs. The channels that were located in these regions encompassed the area of interest, but also outer volumetric areas. The goal of creating an average of the channels was to obtain the averaged response of the language areas in the temporal region during the two language paradigms. In order to get an average that reflected only those areas involved in language function in the brain, statistical analysis was used to determine whether or not each channel was contributing information from these areas. 
In order to create the regional average, the channels were divided into two temporal regions, the anterior region (encompassing Broca's area and its right analog) which resided in the front of the temporal region, and the posterior region (encompassing Wernicke's area and its right analog) which resided in the rear of the fronto-temporal region. Each region is comprised of four channels, in the anterior region these channels are (Ch.5, Ch.6, Ch.7, and Ch.8), and in the posterior region these channels are (Ch.1, Ch.2, Ch.3, Ch.4). Two of the channels, channel 4 and channel 5, encompassed not only the language area of interest, but also parts of the motor cortex as well as auditory cortex. These two channels were excluded due to the fact that the information contained in these channels related not just to the language areas of the brain, but other areas as well. Statistical analysis was used to determine whether or not these channels were actually contributing information regarding to the language areas of the brain as described below.

A two tailed t-test was used to obtain the p-values between the individual channel and the overall mean (described in section 3.5). Once the average for a given region was calculated, an activation analysis (described in section 3.4.1), functional connectivity analysis ( described in section 3.4.2), and lateralization analysis (described in section 3.4.3) was performed for both language paradigms.

The regional average contains channels 6,7 , and 8 from the anterior region, and channels 1,2 , and 3 from the posterior region. Channels 4 and 5 were excluded from the regional analysis to circumvent the volume conduction effect, since when a single source is shared by two different detectors it potentially induces a volume conduction effect. Aside from the potential volumetric conduction these channels could introduce, statistical analysis was used to confirm that these two channels (both in the left and right hemisphere) were significantly different from the other 3 
channels in both the anterior and posterior region. Two methods were used to determine to exclude channels 4 and 5 . The first was by comparison of the average of all the channels to the average of the individual channel for each of the three stimuli for both the word expression paradigm, as well as the word reception paradigm by means of a two tailed t-test $(\alpha=0.05)$. All channels except channels 4 and 5 showed not to be statistically significantly different when compared to their respective regional average (i.e channels in a particular region compared to the average of all channels comprising that particular region) (i.e $\mathrm{p}>0.05$ ), but channels 4 and 5 were found to be statistically significantly different (i.e $p<0.05$ ) from their respective regional means ((both in the left and right hemisphere) (for both 4 channel average (including Ch.4, Ch.5, and 3 channel average (excluding Ch.4, Ch.5)). For the 4 channel average, the outcome was to reject null hypothesis that channels 4 and 5 were equal to regional mean, and accept alternative hypothesis that channels 4 and 5's means were significantly different from the regional average. Once the t-test had been performed the standard deviations of the regional averages were also compared to both the regional averages containing channels 4 and 5( 4 channel average), as well as the regional averages with these channels removed( i.e the three channel regional mean and standard deviation for each of the four regions). It was found that the 3 channel regional average standard deviations which excluded channels 4 and 5 were smaller than the 4 channel regional average standard deviations containing channels 4 and 5( See Tables D1-D12). 


\begin{tabular}{|c|c|c|c|c|}
\hline $\begin{array}{l}4 \text { Channel Regional } \\
\text { Average }\end{array}$ & $\begin{array}{l}\text { Oxy-Hemoglobin( } \\
\text { HbO) }\end{array}$ & Word Generation & Jaw Movement & Rest \\
\hline \multirow[t]{2}{*}{ Left Anterior } & Mean & 1.14E-05 & $-1.78 \mathrm{E}-06$ & $4.75 \mathrm{E}-06$ \\
\hline & Std. Dev. & $4.54 \mathrm{E}-06$ & $2.63 \mathrm{E}-06$ & $3.02 \mathrm{E}-06$ \\
\hline \multirow[t]{2}{*}{ Left Posterior } & Mean & 7.53E-06 & $9.30 \mathrm{E}-06$ & $4.45 \mathrm{E}-06$ \\
\hline & Std. Dev. & $3.68 \mathrm{E}-06$ & $1.20 \mathrm{E}-06$ & $1.51 \mathrm{E}-06$ \\
\hline \multirow[t]{2}{*}{ Right Anterior } & Mean & $5.22 \mathrm{E}-06$ & $5.88 \mathrm{E}-06$ & $5.35 \mathrm{E}-06$ \\
\hline & Std. Dev. & $3.28 \mathrm{E}-06$ & $2.46 \mathrm{E}-06$ & $3.24 \mathrm{E}-06$ \\
\hline \multirow[t]{2}{*}{ Right Posterior } & Mean & 6.90E-06 & $8.51 \mathrm{E}-06$ & $3.62 \mathrm{E}-06$ \\
\hline & Std. Dev. & $3.74 \mathrm{E}-06$ & $1.12 \mathrm{E}-06$ & 1.17E-06 \\
\hline \multicolumn{5}{|l|}{$\begin{array}{l}3 \text { Channel Regional } \\
\text { Average }\end{array}$} \\
\hline \multirow[t]{2}{*}{ Left Anterior } & Mean & 9.03E-06 & $-1.85 E-06$ & $4.26 \mathrm{E}-06$ \\
\hline & Std. Dev. & 3.75E-07 & $2.22 \mathrm{E}-08$ & $2.91 \mathrm{E}-08$ \\
\hline \multirow[t]{2}{*}{ Left Posterior } & Mean & 7.51E-06 & 8.97E-06 & $3.67 \mathrm{E}-06$ \\
\hline & Std. Dev. & 3.70E-07 & $1.36 \mathrm{E}-07$ & $1.26 \mathrm{E}-07$ \\
\hline \multirow[t]{2}{*}{ Right Anterior } & Mean & 5.31E-06 & $5.72 \mathrm{E}-06$ & $5.13 \mathrm{E}-06$ \\
\hline & Std. Dev. & $3.56 \mathrm{E}-07$ & $2.73 \mathrm{E}-07$ & $3.03 \mathrm{E}-07$ \\
\hline \multirow[t]{2}{*}{ Right Posterior } & Mean & 7.26E-06 & 8.97E-06 & $3.30 \mathrm{E}-06$ \\
\hline & Std. Dev. & 3.61E-07 & $9.45 \mathrm{E}-07$ & $1.29 \mathrm{E}-07$ \\
\hline
\end{tabular}

Table D1. A table of the mean and standard deviation of the 4 channel regional average and the 3 channel regional average for $\mathrm{HbO}$ during the Word Expression Paradigm.

\begin{tabular}{|c|c|c|c|c|}
\hline $\begin{array}{l}4 \text { Channel Regional } \\
\text { Average }\end{array}$ & $\begin{array}{l}\text { Deoxy } \\
\text { hemoglobin(HbR) }\end{array}$ & Word Generation & Jaw Movement & Rest \\
\hline \multirow[t]{2}{*}{ Left Anterior } & Mean & $-5.66 \mathrm{E}-06$ & $-9.28 \mathrm{E}-06$ & $-1.47 \mathrm{E}-06$ \\
\hline & Std. Dev. & $5.83 \mathrm{E}-06$ & $4.89 E-06$ & $1.28 \mathrm{E}-06$ \\
\hline \multirow[t]{2}{*}{ Left Posterior } & Mean & $-1.75 \mathrm{E}-06$ & $-2.76 \mathrm{E}-06$ & $-1.05 E-06$ \\
\hline & Std. Dev. & $1.71 \mathrm{E}-06$ & $9.62 \mathrm{E}-07$ & $5.89 \mathrm{E}-07$ \\
\hline \multirow[t]{2}{*}{ Right Anterior } & Mean & $-6.72 \mathrm{E}-06$ & $-5.58 \mathrm{E}-06$ & $-8.48 \mathrm{E}-07$ \\
\hline & Std. Dev. & $5.32 \mathrm{E}-06$ & $4.66 \mathrm{E}-06$ & $1.04 \mathrm{E}-06$ \\
\hline \multirow[t]{2}{*}{ Right Posterior } & Mean & $-6.06 \mathrm{E}-07$ & $-2.25 \mathrm{E}-06$ & $-5.47 E-07$ \\
\hline & Std. Dev. & $1.50 \mathrm{E}-06$ & $7.00 \mathrm{E}-07$ & $3.91 \mathrm{E}-07$ \\
\hline \multicolumn{5}{|l|}{$\begin{array}{l}3 \text { Channel Regional } \\
\text { Average }\end{array}$} \\
\hline \multirow[t]{2}{*}{ Left Anterior } & Mean & $-6.63 E-06$ & $-8.24 \mathrm{E}-06$ & $-1.12 \mathrm{E}-06$ \\
\hline & Std. Dev. & $6.05 \mathrm{E}-07$ & $5.33 \mathrm{E}-07$ & $1.34 \mathrm{E}-07$ \\
\hline \multirow[t]{2}{*}{ Left Posterior } & Mean & $-9.49 \mathrm{E}-07$ & $-1.99 \mathrm{E}-06$ & $-7.36 \mathrm{E}-07$ \\
\hline & Std. Dev. & $1.33 \mathrm{E}-07$ & $8.42 \mathrm{E}-07$ & 5.53E-07 \\
\hline Right Anterior & Mean & $-6.65 E-06$ & $-6.08 \mathrm{E}-06$ & $-1.28 \mathrm{E}-06$ \\
\hline
\end{tabular}




\begin{tabular}{|l|l|r|r|r|}
\hline & Std. Dev. & $5.45 \mathrm{E}-07$ & $4.59 \mathrm{E}-07$ & $8.99 \mathrm{E}-07$ \\
\hline \multirow{2}{*}{ Right Posterior } & Mean & $-2.69 \mathrm{E}-07$ & $-1.64 \mathrm{E}-06$ & $-6.08 \mathrm{E}-07$ \\
\cline { 2 - 5 } & Std. Dev. & $1.29 \mathrm{E}-07$ & $4.43 \mathrm{E}-07$ & $3.95 \mathrm{E}-07$ \\
\hline
\end{tabular}

Table D2. A table of the mean and standard deviation of the 4 channel regional average and the 3 channel regional average for $\mathrm{HbR}$ during the Word Expression Paradigm.

\begin{tabular}{|c|c|c|c|c|}
\hline $\begin{array}{l}4 \text { Channel Regional } \\
\text { Average }\end{array}$ & $\begin{array}{l}\text { Total Hemoglobin } \\
(\mathrm{HbT})\end{array}$ & Word Generation & Jaw Movement & Rest \\
\hline \multirow[t]{2}{*}{ Left Anterior } & Mean & $6.04 \mathrm{E}-06$ & $-1.13 \mathrm{E}-05$ & $3.01 \mathrm{E}-06$ \\
\hline & Std. Dev. & $5.96 \mathrm{E}-06$ & $4.92 \mathrm{E}-06$ & $2.34 \mathrm{E}-06$ \\
\hline \multirow[t]{2}{*}{ Left Posterior } & Mean & $5.73 \mathrm{E}-06$ & $6.54 \mathrm{E}-06$ & $3.62 \mathrm{E}-06$ \\
\hline & Std. Dev. & $2.48 \mathrm{E}-06$ & $9.87 \mathrm{E}-07$ & $1.41 \mathrm{E}-06$ \\
\hline \multirow[t]{2}{*}{ Right Anterior } & Mean & $-1.27 \mathrm{E}-06$ & $6.54 \mathrm{E}-06$ & $4.88 \mathrm{E}-06$ \\
\hline & Std. Dev. & $5.05 \mathrm{E}-06$ & $6.57 \mathrm{E}-06$ & 3.69E-06 \\
\hline \multirow[t]{2}{*}{ Right Posterior } & Mean & $6.26 \mathrm{E}-06$ & $6.33 \mathrm{E}-06$ & $3.23 E-06$ \\
\hline & Std. Dev. & $2.80 \mathrm{E}-06$ & $9.43 \mathrm{E}-07$ & $1.04 \mathrm{E}-06$ \\
\hline \multicolumn{5}{|l|}{$\begin{array}{l}3 \text { Channel Regional } \\
\text { Average }\end{array}$} \\
\hline \multirow[t]{2}{*}{ Left Anterior } & Mean & $2.69 \mathrm{E}-06$ & $-1.05 \mathrm{E}-05$ & $2.98 \mathrm{E}-06$ \\
\hline & Std. Dev. & $6.33 \mathrm{E}-07$ & $5.41 \mathrm{E}-07$ & 1.87E-07 \\
\hline \multirow[t]{2}{*}{ Left Posterior } & Mean & $6.49 \mathrm{E}-06$ & $7.03 \mathrm{E}-06$ & $3.16 \mathrm{E}-06$ \\
\hline & Std. Dev. & $2.83 \mathrm{E}-07$ & $1.09 \mathrm{E}-07$ & $1.46 \mathrm{E}-07$ \\
\hline \multirow[t]{2}{*}{ Right Anterior } & Mean & $-1.09 \mathrm{E}-06$ & $-1.17 \mathrm{E}-06$ & 7.37E-06 \\
\hline & Std. Dev. & 5.57E-07 & $6.84 \mathrm{E}-07$ & $3.42 \mathrm{E}-07$ \\
\hline \multirow[t]{2}{*}{ Right Posterior } & Mean & $6.95 \mathrm{E}-06$ & 7.37E-06 & $2.93 \mathrm{E}-06$ \\
\hline & Std. Dev. & $2.85 \mathrm{E}-06$ & $9.12 \mathrm{E}-07$ & $1.39 \mathrm{E}-06$ \\
\hline
\end{tabular}

Table D3. A table of the mean and standard deviation of the 4 channel regional average and the 3 channel regional average for $\mathrm{HbT}$ during the Word Expression Paradigm. 


\begin{tabular}{|c|c|c|c|c|}
\hline $\begin{array}{l}4 \text { Channel Regional } \\
\text { Average }\end{array}$ & $\begin{array}{l}\text { Oxy-Hemoglobin } \\
\text { (HbO) }\end{array}$ & Forward Audio & Reverse Audio & Rest \\
\hline \multirow[t]{2}{*}{ Left Anterior } & Mean & $4.95 \mathrm{E}-07$ & $-1.80 \mathrm{E}-06$ & $-3.44 \mathrm{E}-07$ \\
\hline & Std. Dev. & $1.39 \mathrm{E}-06$ & $1.01 \mathrm{E}-06$ & $1.03 \mathrm{E}-06$ \\
\hline \multirow[t]{2}{*}{ Left Posterior } & Mean & $-1.55 \mathrm{E}-06$ & $-2.58 \mathrm{E}-06$ & $1.36 \mathrm{E}-07$ \\
\hline & Std. Dev. & $1.47 \mathrm{E}-06$ & 7.32E-07 & $1.28 \mathrm{E}-06$ \\
\hline \multirow[t]{2}{*}{ Right Anterior } & Mean & $7.46 \mathrm{E}-07$ & $-8.98 \mathrm{E}-07$ & $-7.06 \mathrm{E}-07$ \\
\hline & Std. Dev. & $1.02 \mathrm{E}-06$ & $9.52 \mathrm{E}-07$ & $6.41 \mathrm{E}-07$ \\
\hline \multirow[t]{2}{*}{ Right Posterior } & Mean & $-4.47 \mathrm{E}-07$ & $-2.73 E-06$ & $-7.46 \mathrm{E}-07$ \\
\hline & Std. Dev. & $8.95 \mathrm{E}-07$ & 4.71E-07 & $6.11 \mathrm{E}-07$ \\
\hline \multicolumn{5}{|l|}{$\begin{array}{l}3 \text { Channel Regional } \\
\text { Average }\end{array}$} \\
\hline \multirow[t]{2}{*}{ Left Anterior } & Mean & $3.12 \mathrm{E}-07$ & $-2.26 \mathrm{E}-06$ & $-5.50 \mathrm{E}-07$ \\
\hline & Std. Dev. & $1.48 \mathrm{E}-08$ & $1.30 \mathrm{E}-08$ & $1.10 \mathrm{E}-08$ \\
\hline \multirow[t]{2}{*}{ Left Posterior } & Mean & $-1.80 \mathrm{E}-06$ & $-3.00 \mathrm{E}-06$ & $1.08 \mathrm{E}-07$ \\
\hline & Std. Dev. & $1.73 \mathrm{E}-08$ & $8.47 \mathrm{E}-08$ & $1.52 \mathrm{E}-08$ \\
\hline \multirow[t]{2}{*}{ Right Anterior } & Mean & $4.41 \mathrm{E}-07$ & $-8.64 \mathrm{E}-07$ & $-7.40 \mathrm{E}-07$ \\
\hline & Std. Dev. & 9.89E-08 & 1.09E-08 & 7.61E-08 \\
\hline \multirow[t]{2}{*}{ Right Posterior } & Mean & $-7.25 \mathrm{E}-07$ & $-2.66 \mathrm{E}-06$ & $-8.00 \mathrm{E}-07$ \\
\hline & Std. Dev. & $9.00 \mathrm{E}-08$ & 3.7E-07 & $6.54 \mathrm{E}-07$ \\
\hline
\end{tabular}

Table D4. A table of the mean and standard deviation of the 4 channel regional average and the 3 channel regional average for $\mathrm{HbO}$ during the Word Reception Paradigm.

\begin{tabular}{|c|c|c|c|c|}
\hline $\begin{array}{l}4 \text { Channel Regional } \\
\text { Average }\end{array}$ & $\begin{array}{l}\text { Deoxy- } \\
\text { Hemoglobin } \\
\text { (HbR) }\end{array}$ & Forward Audio & Reverse Audio & Rest \\
\hline \multirow[t]{2}{*}{ Left Anterior } & Mean & $3.03 \mathrm{E}-08$ & $2.90 \mathrm{E}-07$ & $8.16 \mathrm{E}-09$ \\
\hline & Std. Dev. & $2.18 \mathrm{E}-07$ & $2.27 \mathrm{E}-07$ & $3.05 E-07$ \\
\hline \multirow[t]{2}{*}{ Left Posterior } & Mean & $-1.73 E-08$ & 7.13E-07 & $4.73 \mathrm{E}-07$ \\
\hline & Std. Dev. & $4.86 \mathrm{E}-07$ & $3.57 \mathrm{E}-07$ & $3.26 \mathrm{E}-07$ \\
\hline \multirow[t]{2}{*}{ Right Anterior } & Mean & $1.24 \mathrm{E}-07$ & $6.64 \mathrm{E}-07$ & 5.33E-07 \\
\hline & Std. Dev. & $2.37 \mathrm{E}-07$ & $2.74 \mathrm{E}-07$ & $1.42 \mathrm{E}-07$ \\
\hline \multirow[t]{2}{*}{ Right Posterior } & Mean & 4.87E-07 & 9.34E-07 & $2.61 \mathrm{E}-07$ \\
\hline & Std. Dev. & 1.19E-07 & $1.44 \mathrm{E}-07$ & $2.56 \mathrm{E}-07$ \\
\hline \multicolumn{5}{|l|}{$\begin{array}{l}3 \text { Channel Regional } \\
\text { Average }\end{array}$} \\
\hline \multirow[t]{2}{*}{ Left Anterior } & Mean & $3.24 \mathrm{E}-07$ & $1.01 \mathrm{E}-06$ & 4.99E-07 \\
\hline & Std. Dev. & $4.82 \mathrm{E}-07$ & 5.33E-08 & $1.35 \mathrm{E}-08$ \\
\hline \multirow[t]{2}{*}{ Left Posterior } & Mean & $6.01 \mathrm{E}-08$ & 2.57E-07 & $-6.42 E-08$ \\
\hline & Std. Dev. & $1.33 \mathrm{E}-08$ & $8.43 \mathrm{E}-08$ & $5.54 \mathrm{E}-08$ \\
\hline Right Anterior & Mean & $1.16 \mathrm{E}-07$ & $6.76 \mathrm{E}-07$ & $6.88 \mathrm{E}-07$ \\
\hline
\end{tabular}




\begin{tabular}{|l|l|r|r|r|}
\hline & Std. Dev. & $5.45 \mathrm{E}-08$ & $4.59 \mathrm{E}-08$ & $8.99 \mathrm{E}-07$ \\
\hline \multirow{2}{*}{ Right Posterior } & Mean & $4.55 \mathrm{E}-07$ & $8.19 \mathrm{E}-07$ & $2.07 \mathrm{E}-07$ \\
\cline { 2 - 5 } & Std. Dev. & $1.29 \mathrm{E}-07$ & $4.44 \mathrm{E}-08$ & $3.96 \mathrm{E}-08$ \\
\hline
\end{tabular}

Table D5. A table of the mean and standard deviation of the 4 channel regional average and the 3 channel regional average for HbR during the Word Reception Paradigm.

\begin{tabular}{|c|c|c|c|c|}
\hline $\begin{array}{l}4 \text { Channel Regional } \\
\text { Average }\end{array}$ & $\begin{array}{l}\text { Total Hemoglobin } \\
\text { (HbT) }\end{array}$ & Forward Audio & Reverse Audio & Rest \\
\hline \multirow[t]{2}{*}{ Left Anterior } & Mean & $2.69 \mathrm{E}-08$ & $2.91 \mathrm{E}-07$ & $2.35 \mathrm{E}-08$ \\
\hline & Std. Dev. & $2.19 \mathrm{E}-07$ & $2.30 \mathrm{E}-07$ & 3.03E-07 \\
\hline \multirow{2}{*}{ Left Posterior } & Mean & $-1.17 \mathrm{E}-08$ & $6.85 \mathrm{E}-07$ & $4.72 \mathrm{E}-07$ \\
\hline & Std. Dev. & $4.90 \mathrm{E}-07$ & $3.92 \mathrm{E}-07$ & $3.12 \mathrm{E}-07$ \\
\hline \multirow{2}{*}{ Right Anterior } & Mean & $1.14 \mathrm{E}-07$ & $6.68 \mathrm{E}-07$ & $5.28 \mathrm{E}-07$ \\
\hline & Std. Dev. & $2.31 \mathrm{E}-07$ & $2.76 \mathrm{E}-07$ & $1.38 \mathrm{E}-07$ \\
\hline \multirow{2}{*}{ Right Posterior } & Mean & $4.82 \mathrm{E}-07$ & 9.44E-07 & 2.80E-07 \\
\hline & Std. Dev. & $1.14 \mathrm{E}-07$ & $1.32 \mathrm{E}-07$ & $2.65 \mathrm{E}-07$ \\
\hline \multicolumn{5}{|l|}{$\begin{array}{l}3 \text { Channel Regional } \\
\text { Average }\end{array}$} \\
\hline \multirow[t]{2}{*}{ Left Anterior } & Mean & $3.28 \mathrm{E}-07$ & $9.82 \mathrm{E}-07$ & 5.13E-07 \\
\hline & Std. Dev. & $4.88 \mathrm{E}-07$ & $4.03 \mathrm{E}-07$ & $3.50 \mathrm{E}-07$ \\
\hline \multirow[t]{2}{*}{ Left Posterior } & Mean & $5.62 \mathrm{E}-08$ & $2.64 \mathrm{E}-07$ & $-5.22 \mathrm{E}-08$ \\
\hline & Std. Dev. & $2.15 \mathrm{E}-07$ & $2.90 \mathrm{E}-07$ & $3.08 \mathrm{E}-07$ \\
\hline \multirow[t]{2}{*}{ Right Anterior } & Mean & $1.03 \mathrm{E}-07$ & $6.82 \mathrm{E}-07$ & $6.75 \mathrm{E}-07$ \\
\hline & Std. Dev. & $1.86 \mathrm{E}-07$ & $2.41 \mathrm{E}-07$ & $1.73 \mathrm{E}-07$ \\
\hline \multirow[t]{2}{*}{ Right Posterior } & Mean & $4.49 \mathrm{E}-07$ & 8.33E-07 & $2.20 \mathrm{E}-07$ \\
\hline & Std. Dev. & $1.03 \mathrm{E}-07$ & $1.36 \mathrm{E}-07$ & $1.98 \mathrm{E}-07$ \\
\hline
\end{tabular}

Table D6. A table of the mean and standard deviation of the 4 channel regional average and the 3 channel regional average for HbT during the Word Reception Paradigm. 
Tables D7-D12 are given to show the p-values when comparing channels 4 and 5 to their respective four channel means. Channels 4 and 5 in both the left and right hemispheres of the fronto-temporal regions show p-values less than 0.05 . This means that these means are to be considered statistically significantly different from the regional average, and hence were removed from regional average. P-values from those means that are considered statistically significantly different are highlighted in red.

WORD EXPRESSION PARADIGM p-values (Channels vs. Mean of all four channels) (HbO)

\begin{tabular}{|l|l|l|l|l|l|l|l|l|}
\hline & L1 & L2 & L3 & L4 & R1 & R2 & R3 & R4 \\
\hline W.G & 0.1333 & 0.1238 & 0.1132 & 0.0000 & 0.5328 & 0.1626 & 0.1922 & 0.0000 \\
\hline J.M & 0.6465 & 0.0813 & 0.8897 & 0.0000 & 0.4714 & 0.4301 & 0.3365 & 0.0020 \\
\hline R & 0.2641 & 0.2588 & 0.6311 & 0.0000 & 0.7313 & 0.3796 & 0.2323 & 0.0029 \\
\hline & & & & & & & & \\
\hline & L5 & L6 & L7 & L8 & R5 & R6 & R7 & R8 \\
\hline W.G & 0.0000 & 0.1168 & 0.6817 & 0.6218 & 0.0006 & 0.3810 & 0.7199 & 0.6263 \\
\hline J.M & 0.0001 & 0.3654 & 0.1942 & 0.9590 & 0.0247 & 0.1171 & 0.2127 & 0.2665 \\
\hline R & 0.0000 & 0.6003 & 0.1452 & 0.7470 & 0.0348 & 0.3036 & 0.4068 & 0.1203 \\
\hline
\end{tabular}

Table D7. A table showing the results of the t-test for the Word Expression Paradigm when comparing each individual channel to the overall regional average for each stimuli ( FA-Forward Audio, RA- Reverse Audio, R-Rest) for HbO. P values greater than 0.05 should not be considered statistically different.

WORD EXPRESSION PARADIGM p-values (Channels vs. Mean of all four channels) (HbR)

\begin{tabular}{|l|l|l|l|l|l|l|l|l|}
\hline & L1 & L2 & L3 & L4 & R1 & R2 & R3 & R4 \\
\hline W.G & 0.3300 & 0.5327 & 0.1054 & 0.0001 & 0.2163 & 0.9354 & 0.3358 & 0.0000 \\
\hline J.M & 0.7277 & 0.2732 & 0.9595 & 0.0000 & 0.6114 & 0.3449 & 0.1382 & 0.0000 \\
\hline R & 0.9928 & 0.5404 & 0.0873 & 0.0000 & 0.1274 & 0.2895 & 0.8563 & 0.0056 \\
\hline & & & & & & & & \\
\hline & L5 & L6 & L7 & L8 & R5 & R6 & R7 & R8 \\
\hline W.G & 0.0232 & 0.5895 & 0.7001 & 0.9903 & 0.0000 & 0.4880 & 0.7804 & 0.1854 \\
\hline J.M & 0.0028 & 0.8057 & 0.5537 & 0.1252 & 0.0002 & 0.9200 & 0.8284 & 0.3610 \\
\hline R & 0.0001 & 0.1346 & 0.0692 & 0.3895 & 0.0001 & 0.9339 & 0.2975 & 0.2661 \\
\hline
\end{tabular}

Table D8. A table showing the results of the t-test for the Word Expression Paradigm when comparing each individual channel to the overall regional average for each stimuli ( W.G-Word Expression, J.M- Jaw Movement, R-Rest) for HbR. P values greater than 0.05 should not be considered statistically different. 
WORD EXPRESSION PARADIGM p-values (Channels vs. Mean of all four channels) (HbT)

\begin{tabular}{|l|l|l|l|l|l|l|l|l|}
\hline & L1 & L2 & L3 & L4 & R1 & R2 & R3 & R4 \\
\hline W.G & 0.2427 & 0.8218 & 0.8975 & 0.0019 & 0.3279 & 0.3281 & 0.3268 & 0.0003 \\
\hline J.M & 0.1952 & 0.6456 & 0.3303 & 0.0000 & 0.1192 & 0.7774 & 0.7243 & 0.0000 \\
\hline R & 0.3252 & 0.3252 & 0.9101 & 0.0037 & 0.6875 & 0.9040 & 0.1452 & 0.0000 \\
\hline & & & & & & & & \\
\hline & L5 & L6 & L7 & L8 & R5 & R6 & R7 & R8 \\
\hline W.G & 0.0106 & 0.5726 & 0.2871 & 0.0607 & 0.0000 & 0.3290 & 0.3223 & 0.3250 \\
\hline J.M & 0.0000 & 0.7425 & 0.2426 & 0.8483 & 0.0000 & 0.3279 & 0.2860 & 0.9344 \\
\hline R & 0.0087 & 0.1174 & 0.8726 & 0.1922 & 0.0001 & 0.2239 & 0.1215 & 0.9964 \\
\hline
\end{tabular}

Table D9. A table showing the results of the t-test for the Word Expression Paradigm when comparing each individual channel to the overall regional average for each stimuli ( W.G-Word Expression, J.M- Jaw Movement, R-Rest) for HbT. P values greater than 0.05 should not be considered statistically different.

WORD RECEPTION PARADIGM p-values (Channels vs. Mean of all four channels) (HbO)

\begin{tabular}{|l|l|l|l|l|l|l|l|l|}
\hline & L1 & L2 & L3 & L4 & R1 & R2 & R3 & R4 \\
\hline F.A & 0.2427 & 0.8218 & 0.8975 & 0.0019 & 0.2482 & 0.2082 & 0.5940 & 0.0001 \\
\hline R.A & 0.1952 & 0.6456 & 0.3303 & 0.0000 & 0.5748 & 0.6726 & 0.8079 & 0.0000 \\
\hline R & 0.3252 & 0.3252 & 0.9101 & 0.0037 & 0.5522 & 0.4711 & 0.1218 & 0.0008 \\
\hline & & & & & & & & \\
\hline & L5 & L6 & L7 & L8 & R5 & R6 & R7 & R8 \\
\hline F.A & 0.0000 & 0.1929 & 0.6488 & 2.7537 & 0.0001 & 0.9599 & 0.6190 & 0.8434 \\
\hline R.A & 0.0000 & 0.6877 & 0.3670 & 0.4642 & 0.0000 & 0.2538 & 0.2660 & 0.3342 \\
\hline R & 0.0000 & 0.1706 & 0.7132 & 0.5743 & 0.0000 & 0.7821 & 0.2249 & 0.6122 \\
\hline
\end{tabular}

Table D10. A table showing the results of the t-test for the Word Reception Paradigm when comparing each individual channel to the overall regional average for each stimuli ( FA-Forward Audio, RA- Reverse Audio, R-Rest) for HbO. P values greater than 0.05 should not be considered statistically different.

WORD RECEPTION PARADIGM p-values ( Channels vs. Mean of all four channels) (HbR)

\begin{tabular}{|l|l|l|l|l|l|l|l|l|}
\hline & L1 & L2 & L3 & L4 & R1 & R2 & R3 & R4 \\
\hline F.A & 0.5108 & 0.5162 & 0.3424 & 0.0000 & 0.1083 & 0.1429 & 0.4409 & 0.0000 \\
\hline R.A & 0.7586 & 0.2825 & 0.2936 & 0.0031 & 0.1480 & 0.3096 & 0.9365 & 0.0000 \\
\hline R & 0.8778 & 0.8082 & 0.6459 & 0.0018 & 0.6878 & 0.2502 & 0.5938 & 0.0001 \\
\hline & & & & & & & & \\
\hline & L5 & L6 & L7 & L8 & R5 & R6 & R7 & R8 \\
\hline F.A & 0.0000 & 0.6267 & 0.9985 & 0.8580 & 0.0000 & 0.6627 & 0.8456 & 0.7481 \\
\hline R.A & 0.0000 & 0.2269 & 0.7200 & 0.5812 & 0.0000 & 0.9467 & 0.1073 & 0.6581 \\
\hline R & 0.0004 & 0.1824 & 0.5506 & 0.9074 & 0.0000 & 0.3976 & 0.6094 & 0.9608 \\
\hline
\end{tabular}

Table D11. A table showing the results of the t-test for the Word Reception Paradigm when comparing each individual channel to the overall regional average for each stimuli ( FA-Forward Audio, RA- Reverse Audio, R-Rest) for HbR. P values greater than 0.05 should not be considered statistically different. 
WORD RECEPTION PARADIGM p-values ( Channels vs. Mean of all four channels) (HbT)

\begin{tabular}{|l|l|l|l|l|l|l|l|l|}
\hline & L1 & L2 & L3 & L4 & R1 & R2 & R3 & R4 \\
\hline F.A & 0.3382 & 0.1161 & 0.3240 & 0.0000 & 0.4247 & 0.9796 & 0.1151 & 0.0000 \\
\hline R.A & 0.4485 & 0.3820 & 0.5700 & 0.0000 & 0.1687 & 0.8891 & 0.3685 & 0.0000 \\
\hline R & 0.3256 & 0.3209 & 0.2135 & 0.0000 & 0.3144 & 0.3822 & 0.2456 & 0.0000 \\
\hline & & & & & & & & \\
\hline & L5 & L6 & L7 & L8 & R5 & R6 & R7 & R8 \\
\hline F.A & 0.0000 & 0.3457 & 0.4599 & 0.1450 & 0.0010 & 0.3898 & 0.2029 & 0.8519 \\
\hline R.A & 0.0000 & 0.6049 & 0.8190 & 0.4973 & 0.0000 & 0.9104 & 0.1028 & 0.1862 \\
\hline R & 0.0008 & 0.3269 & 0.3252 & 0.3910 & 0.0455 & 0.3314 & 0.3545 & 0.7100 \\
\hline
\end{tabular}

Table D12. A table showing the results of the t-test for the Word Reception Paradigm when comparing each individual channel to the overall regional average for each stimuli ( FA-Forward Audio, RA- Reverse Audio, R-Rest) for HbT. P values greater than 0.05 should not be considered statistically different.

Tables D13-D18 are given to show the p-values when comparing the three channel mean to individual channels with channels 4 and 5 removed. Channels 4 and 5 in both the left and right hemispheres of the fronto-temporal regions showed p-values less than 0.05 ( Tables. This means that these means are to be considered statistically significantly different from the regional average, and hence were removed from regional average. P-values from those means that are considered statistically significantly different are highlighted in red.

WORD EXPRESSION PARADIGM p-values (Channels vs. Mean of all three channels) (HbO)

\begin{tabular}{|c|c|c|c|c|c|c|}
\hline & $\mathrm{L} 1$ & $\mathrm{~L} 2$ & L3 & $\mathrm{R} 1$ & $\mathrm{R} 2$ & R3 \\
\hline W.G & 0.709 & 0.225 & 0.257 & 0.213 & 0.357 & 0.463 \\
\hline J.M & 0.093 & 0.274 & 0.791 & 0.639 & 0.720 & 0.320 \\
\hline $\mathrm{R}$ & 0.162 & 0.732 & 0.095 & 0.461 & 0.091 & 0.159 \\
\hline & L6 & L7 & L8 & R6 & R7 & R8 \\
\hline W.G & 0.202 & 0.840 & 0.438 & 0.553 & 0.583 & 0.611 \\
\hline J.M & 0.366 & 0.791 & 0.331 & 0.691 & 0.085 & 0.859 \\
\hline$R$ & 0.921 & 0.130 & 0.821 & 0.300 & 0.387 & 0.112 \\
\hline
\end{tabular}

Table D13. A table showing the results of the t-test for the Word Expression Paradigm when comparing each individual channel to the overall regional average for each stimuli ( W.G-Word generation, J.M-Jaw Movement, R-Rest) for HbO. P values greater than 0.05 should not be considered statistically different. 
WORD EXPRESSION PARADIGM p-values (Channels vs. Mean of all three channels) (HbR)

\begin{tabular}{|l|r|r|r|r|r|r|r|}
\hline & \multicolumn{1}{l|}{ L1 } & \multicolumn{1}{l|}{ L2 } & \multicolumn{1}{l|}{ L3 } & & \multicolumn{2}{l|}{ R1 } & \multicolumn{2}{l|}{ R2 } & \multicolumn{1}{l|}{ R3 } \\
\hline W.G & 0.220 & 0.858 & 0.069 & & 0.053 & 0.103 & 0.407 \\
\hline J.M & 0.510 & 0.298 & 0.763 & & 0.663 & 0.727 & 0.523 \\
\hline R & 0.227 & 0.187 & 0.322 & & 0.785 & 0.423 & 0.685 \\
\hline & & & & & & & \\
\hline & L6 & L7 & L8 & & R6 & R7 & \multicolumn{1}{l|}{ R8 } \\
\hline W.G & 0.454 & 0.113 & 0.071 & & 0.060 & 0.749 & 0.179 \\
\hline J.M & 0.150 & 0.212 & 0.511 & & 0.852 & 0.458 & 0.533 \\
\hline R & 0.330 & 0.488 & 0.885 & & 0.187 & 0.386 & 0.139 \\
\hline
\end{tabular}

Table D14. A table showing the results of the t-test for the Word Expression Paradigm when comparing each individual channel to the overall regional average for each stimuli ( W.G-Word generation, J.M-Jaw Movement, R-Rest) for HbR. P values greater than 0.05 should not be considered statistically different.

WORD EXPRESSION PARADIGM p-values (Channels vs. Mean of all three channels) (HbT)

\begin{tabular}{|c|c|c|c|c|c|c|}
\hline & L1 & $\mathrm{L} 2$ & L3 & R1 & $\mathrm{R} 2$ & R3 \\
\hline W.G & 0.949 & 0.515 & 0.764 & 0.593 & 0.816 & 0.193 \\
\hline J.M & 0.064 & 0.216 & 0.175 & 0.882 & 0.653 & 0.437 \\
\hline $\mathrm{R}$ & 0.099 & 0.491 & 0.340 & 0.291 & 0.284 & 0.307 \\
\hline & L6 & L7 & L8 & R6 & R7 & R8 \\
\hline W.G & 0.230 & 0.025 & 0.036 & 0.709 & 0.847 & 0.183 \\
\hline J.M & 0.286 & 0.013 & 0.890 & 0.348 & 0.005 & 0.496 \\
\hline $\mathrm{R}$ & 0.588 & 0.388 & 0.606 & 0.009 & 0.121 & 0.448 \\
\hline
\end{tabular}

Table D15. A table showing the results of the t-test for the Word Expression Paradigm when comparing each individual channel to the overall regional average for each stimuli ( W.G-Word generation, J.M-Jaw Movement, R-Rest) for HbR. P values greater than 0.05 should not be considered statistically different.

WORD RECEPTION PARADIGM p-values (Channels vs. Mean of all three channels) (HbO)

\begin{tabular}{|c|c|c|c|c|c|c|}
\hline & L1 & L2 & L3 & R1 & $\mathrm{R} 2$ & R3 \\
\hline FA & 0.778 & 0.488 & 0.217 & 0.702 & 0.944 & 0.518 \\
\hline RA & 0.098 & 0.613 & 0.083 & 0.289 & 0.987 & 0.646 \\
\hline $\mathrm{R}$ & 0.533 & 0.254 & 0.135 & 0.949 & 0.349 & 0.319 \\
\hline & L6 & L7 & L8 & R6 & R7 & R8 \\
\hline FA & 0.081 & 0.605 & 0.285 & 0.235 & 0.807 & 0.388 \\
\hline RA & 0.204 & 0.836 & 0.052 & 0.057 & 0.750 & 0.178 \\
\hline $\mathrm{R}$ & 0.402 & 0.160 & 0.125 & 0.567 & 0.262 & 0.943 \\
\hline
\end{tabular}

Table D16. A table showing the results of the t-test for the Word Reception Paradigm when comparing each individual channel to the overall regional average for each stimuli ( FA-Forward Audio, RA- Reverse Audio, R-Rest) for HbO. P values greater than 0.05 should not be considered statistically different. 
WORD RECEPTION PARADIGM p-values ( Channels vs. Mean of all three channels) (HbR)

\begin{tabular}{|c|c|c|c|c|c|c|}
\hline & L1 & $\mathrm{L} 2$ & L3 & $\mathrm{R} 1$ & $\mathrm{R} 2$ & R3 \\
\hline FA & 0.122 & 0.238 & 0.890 & 0.328 & 0.760 & 0.069 \\
\hline RA & 0.286 & 0.118 & 0.276 & 0.468 & 0.107 & 0.813 \\
\hline $\mathrm{R}$ & 0.077 & 0.966 & 0.422 & 0.183 & 0.423 & 0.392 \\
\hline & L6 & L7 & L8 & R6 & R7 & R8 \\
\hline FA & 0.219 & 0.537 & 0.147 & 0.910 & 0.448 & 0.159 \\
\hline RA & 0.110 & 0.185 & 0.683 & 0.575 & 0.550 & 0.061 \\
\hline$R$ & 0.467 & 0.901 & 0.169 & 0.718 & 0.087 & 0.283 \\
\hline
\end{tabular}

Table D17. A table showing the results of the t-test for the Word Reception Paradigm when comparing each individual channel to the overall regional average for each stimuli ( FA-Forward Audio, RA- Reverse Audio, R-Rest) for HbR. P values greater than 0.05 should not be considered statistically different.

WORD RECEPTION PARADIGM p-values ( Channels vs. Mean of all three channels) (HbT)

\begin{tabular}{|l|r|r|r|r|r|r|r|}
\hline & \multicolumn{1}{|l|}{ L1 } & \multicolumn{1}{l|}{ L2 } & \multicolumn{1}{l|}{ L3 } & & \multicolumn{2}{l|}{ R1 } & \multicolumn{2}{l|}{ R2 } & \multicolumn{1}{l|}{ R3 } \\
\hline FA & 0.412 & 0.287 & 0.369 & & 0.06 & 0.088 & 0.705 \\
\hline RA & 0.43 & 0.135 & 0.676 & & 0.922 & 0.688 & 0.762 \\
\hline R & 0.789 & 0.099 & 0.399 & & 0.113 & 0.144 & 0.618 \\
\hline & & & & & & & \\
\hline & L6 & L7 & L8 & & R6 & R7 & R8 \\
\hline FA & 0.119 & 0.814 & 0.412 & & 0.072 & 0.197 & 0.937 \\
\hline RA & 0.481 & 0.207 & 0.629 & & 0.193 & 0.739 & 0.126 \\
\hline R & 0.139 & 0.841 & 0.596 & & 0.324 & 0.179 & 0.936 \\
\hline
\end{tabular}

Table D18. A table showing the results of the t-test for the Word Reception Paradigm when comparing each individual channel to the overall regional average for each stimuli ( FA-Forward Audio, RA- Reverse Audio, R-Rest) for HbT. P values greater than 0.05 should not be considered statistically different.

\section{Appendix E- Word Expression Statistical Analysis Results}

The results from the 3 paired p-value analysis (for word expression paradigm) are given below as tables E1-E3 for the channel-wise analysis, and as tables E4-E6 for the regional analysis. These analyses were performed to determine if activation observed during the three stimuli presented during the word expression paradigm were statistically the same or statistically different from one another. The p-values that are smaller than 0.05 are to be considered statistically significantly different. This is to be interpreted 
as the activation seen during each task differed from one another, which means that each of the three stimuli presented caused a different activation of the brain. For the word expression paradigm, the activation differed across all 16 channels, and all four regions in the temporal region of the brain for each of the three stimuli presented, for $\mathrm{HbO}, \mathrm{HbR}$, and $\mathrm{HbT}$. A detailed analysis of how the three paired p-test was performed is given in section 3.5.2.

\begin{tabular}{|l|r|r|r|}
\hline \multicolumn{4}{|c|}{ HbO P-values for Word Expression Paradigm } \\
\hline Channel & \multicolumn{3}{|c|}{ Stimuli Comparison } \\
\hline & Word v. Jaw & Word v. Rest & Jaw v. Rest \\
\hline L1 & 0.0001 & 0.0000 & 0.0000 \\
\hline L2 & 0.0000 & 0.0000 & 0.0038 \\
\hline L3 & 0.0000 & 0.0005 & 0.0000 \\
\hline L4 & 0.0001 & 0.005 & 0.0000 \\
\hline L5 & 0.0000 & 0.0000 & 0.0000 \\
\hline L6 & 0.0000 & 0.0000 & 0.0000 \\
\hline L7 & 0.0000 & 0.05 & 0.0000 \\
\hline L8 & 0.0000 & 0.0001 & 0.045 \\
\hline R1 & 0.0012 & 0.0000 & 0.0000 \\
\hline R2 & 0.0005 & 0.0000 & 0.0000 \\
\hline R3 & 0.0188 & 0.0009 & 0.0000 \\
\hline R4 & 0.0003 & 0.0000 & 0.0000 \\
\hline R5 & 0.0318 & 0.0000 & 0.0000 \\
\hline R6 & 0.0005 & 0.0000 & 0.0000 \\
\hline R7 & 0.008 & 0.0000 & 0.0000 \\
\hline R8 & 0.0000 & 0.0002 & 0.0503 \\
\hline
\end{tabular}

Table E1: A table of the three paired p-test results for Oxy-hemoglobin $(\mathrm{HbO})$ during the Word Expression Paradigm 


\begin{tabular}{|l|r|r|r|}
\hline \multicolumn{4}{|c|}{ HbR P-values Word Expression Paradigm } \\
\hline Channel & \multicolumn{3}{|c|}{ Stimuli Comparison } \\
\hline & Word v. Jaw & \multicolumn{1}{|c|}{ Word v. Rest } & Jaw v. Rest \\
\hline L1 & 0.0000 & 0.03290 & 0.0000 \\
\hline L2 & 0.0000 & 0.0050 & 0.0000 \\
\hline L3 & 0.0001 & 0.0305 & 0.0000 \\
\hline L4 & 0.0285 & 0.0002 & 0.0000 \\
\hline L5 & 0.0000 & 0.0000 & 0.0000 \\
\hline L6 & 0.0063 & 0.0000 & 0.0000 \\
\hline L7 & 0.0001 & 0.0229 & 0.0000 \\
\hline L8 & 0.0011 & 0.0000 & 0.0000 \\
\hline R1 & 0.0000 & 0.0000 & 0.0000 \\
\hline R2 & 0.0000 & 0.0001 & 0.0000 \\
\hline R3 & 0.0000 & 0.0000 & 0.0000 \\
\hline R4 & 0.0000 & 0.0037 & 0.0000 \\
\hline R5 & 0.0090 & 0.0000 & 0.0000 \\
\hline R6 & 0.0159 & 0.0272 & 0.0000 \\
\hline R7 & 0.0000 & 0.0000 & 0.0000 \\
\hline R8 & 0.0000 & 0.0000 & 0.0000 \\
\hline
\end{tabular}

Table E2: A table of the three paired p-test results for Deoxy-hemoglobin (HbR) during the Word Expression Paradigm.

\begin{tabular}{|l|r|r|r|}
\hline \multicolumn{3}{|c|}{ HbT P-values for Word Expression Paradigm } \\
\hline Channel & \multicolumn{3}{|c|}{ Stimuli Comparison } \\
\hline & Word v. Jaw & Word v.Rest & Jaw v. Rest \\
\hline L1 & 0.0073 & 0.0000 & 0.0000 \\
\hline L2 & 0.0001 & 0.0000 & 0.0001 \\
\hline L3 & 0.0000 & 0.0000 & 0.0000 \\
\hline L4 & 0.0002 & 0.0006 & 0.0000 \\
\hline L5 & 0.0000 & 0.0000 & 0.0000 \\
\hline L6 & 0.0000 & 0.0326 & 0.0000 \\
\hline L7 & 0.0000 & 0.0000 & 0.0000 \\
\hline L8 & 0.0000 & 0.0414 & 0.0001 \\
\hline R1 & 0.0000 & 0.0000 & 0.0000 \\
\hline R2 & 0.005 & 0.0000 & 0.0000 \\
\hline R3 & 0.0000 & 0.0000 & 0.0000 \\
\hline R4 & 0.0000 & 0.0000 & 0.0044 \\
\hline R5 & 0.0043 & 0.0000 & 0.0001 \\
\hline R6 & 0.0000 & 0.0000 & 0.0000 \\
\hline R7 & 0.0000 & 0.0000 & 0.0000 \\
\hline R8 & 0.0002 & 0.0000 & 0.0000 \\
\hline
\end{tabular}

Table E3 :A table of three paired p-test results for Total hemoglobin (HbT) during the Word Expression Paradigm. 


\begin{tabular}{|c|r|r|r|}
\hline \multicolumn{4}{|c|}{ HbO 3 paired P-values for Word Expression Paradigm } \\
\hline Region & \multicolumn{3}{|c|}{ Stimuli Comparison } \\
\hline & Word v. Jaw & Word v. Rest & Jaw v. Rest \\
\hline Left Anterior & 0.000 & 0.000 & 0.000 \\
\hline Left Posterior & 0.005 & 0.000 & 0.000 \\
\hline Right Anterior & 0.000 & 0.000 & 0.000 \\
\hline Right Posterior & 0.001 & 0.000 & 0.000 \\
\hline
\end{tabular}

Table E4: A table of the three paired p-test results for Oxy-hemoglobin (HbO) during the Regional Word Expression Paradigm

\begin{tabular}{|l|r|r|r|}
\hline \multicolumn{4}{|c|}{ HbR 3 paired P-values for Word Expression Paradigm } \\
\hline Region & \multicolumn{3}{|c|}{ Stimuli Comparison } \\
\hline & Word v. Jaw & Word v. Rest & Jaw v. Rest \\
\hline Left Anterior & 0.038 & 0.000 & 0.000 \\
\hline Left Posterior & 0.000 & 0.000 & 0.000 \\
\hline Right Anterior & 0.000 & 0.000 & 0.000 \\
\hline Right Posterior & 0.000 & 0.035 & 0.000 \\
\hline
\end{tabular}

Table E5: A table of the three paired p-test results for Deoxy-hemoglobin (HbR) during the Regional Word Expression Paradigm

\begin{tabular}{|l|r|r|r|}
\hline \multicolumn{4}{|c|}{ HbT 3 paired P-values for Word Expression Paradigm } \\
\hline Region & \multicolumn{3}{|c|}{ Stimuli Comparison } \\
\hline & Word v. Jaw & Word v. Rest & Jaw v. Rest \\
\hline Left Anterior & 0.000 & 0.000 & 0.000 \\
\hline Left Posterior & 0.000 & 0.000 & 0.000 \\
\hline Right Anterior & 0.000 & 0.000 & 0.000 \\
\hline Right Posterior & 0.002 & 0.000 & 0.000 \\
\hline
\end{tabular}

Table E6: A table of the three paired p-test results for Total hemoglobin (HbT) during the Regional Word Expression Paradigm. 


\section{Appendix F- Word Reception Statistical Analysis Results}

The results from the 3 paired p-value analysis (for word reception paradigm) are given below as tables F1-F3 for the channel-wise analysis, and as tables F4-F6 for the regional analysis. These analyses were performed to determine if activation observed during the three stimuli presented during the word reception paradigm were statistically the same or statistically different from one another. The p-values that is smaller than 0.05 are to be considered statistically significantly different. This is to be interpreted as the activation seen during each task differed from one another, which means that each of the three stimuli presented caused a different activation of the brain. For the word reception paradigm, the activation differed across all 16 channels and all four regions in the temporal region of the brain for each of the three stimuli presented, for $\mathrm{HbO}, \mathrm{HbR}$, and $\mathrm{HbT}$. A detailed analysis of how the three paired p-test was performed is given in section 3.5.2.

\begin{tabular}{|l|r|r|r|}
\hline \multicolumn{4}{|c|}{ HbO P values for Word Reception Paradigm } \\
\hline Channel & \multicolumn{3}{|c|}{ Stimuli Comparison } \\
\hline & ${ }^{*}$ FA v. ${ }^{* *}$ RA & ${ }^{*}$ FA v. Rest & ${ }^{* *}$ RA v. Rest \\
\hline L1 & 0.0001 & 0.0000 & 0.0000 \\
\hline L2 & 0.0000 & 0.0015 & 0.0000 \\
\hline L3 & 0.0042 & 0.0000 & 0.0000 \\
\hline L4 & 0.0001 & 0.0000 & 0.0000 \\
\hline L5 & 0.0000 & 0.0360 & 0.0007 \\
\hline L6 & 0.0000 & 0.0006 & 0.0000 \\
\hline L7 & 0.0000 & 0.0117 & 0.0000 \\
\hline L8 & 0.0000 & 0.0116 & 0.0000 \\
\hline R1 & 0.0000 & 0.0024 & 0.0000 \\
\hline R2 & 0.0000 & 0.0014 & 0.0000 \\
\hline R3 & 0.0000 & 0.0000 & 0.0000 \\
\hline R4 & 0.0000 & 0.0000 & 0.0000 \\
\hline R5 & 0.0000 & 0.0000 & 0.0436 \\
\hline R6 & 0.0000 & 0.0000 & 0.0434 \\
\hline R7 & 0.0083 & 0.0000 & 0.0036 \\
\hline R8 & 0.0000 & 0.0000 & 0.0000 \\
\hline \multicolumn{2}{|r|}{${ }^{*}$ FA= Forward Audio, } & $* *$ RA= Reverse Audio \\
\hline
\end{tabular}

Table F1: A table of the three paired p-test results for Oxy-hemoglobin (HbO) during the Word Reception Paradigm. 


\begin{tabular}{|l|r|r|r|}
\hline \multicolumn{3}{|c|}{ HbR P-values for the Word Reception Paradigm } \\
\hline Channel & \multicolumn{3}{|c|}{ Stimuli Comparison } \\
\hline & ${ }^{*}$ FA v. ${ }^{* *}$ RA & *FA v. Rest & ${ }^{* *}$ RA v. Rest \\
\hline L1 & 0.0000 & 0.0000 & 0.0009 \\
\hline L2 & 0.0001 & 0.0012 & 0.0015 \\
\hline L3 & 0.0000 & 0.0000 & 0.0000 \\
\hline L4 & 0.0000 & 0.0005 & 0.0000 \\
\hline L5 & 0.0000 & 0.0000 & 0.0000 \\
\hline L6 & 0.0000 & 0.0000 & 0.0000 \\
\hline L7 & 0.0000 & 0.0000 & 0.0002 \\
\hline L8 & 0.0000 & 0.0014 & 0.0000 \\
\hline R1 & 0.0000 & 0.0000 & 0.0000 \\
\hline R2 & 0.0000 & 0.0000 & 0.0000 \\
\hline R3 & 0.0000 & 0.0000 & 0.0000 \\
\hline R4 & 0.0000 & 0.0000 & 0.0000 \\
\hline R5 & 0.0000 & 0.0051 & 0.0000 \\
\hline R6 & 0.0000 & 0.0017 & 0.0003 \\
\hline R7 & 0.0002 & 0.0005 & 0.0009 \\
\hline R8 & 0.0000 & 0.0000 & 0.0003 \\
\hline \multicolumn{3}{|r|}{${ }^{*}$ FA= Forward Audio, } & $* *$ RA= Reverse Audio \\
\hline
\end{tabular}

Table F2: A table of the three paired p-test results for Deoxy-hemoglobin (HbR) during the Word Reception Paradigm.

\begin{tabular}{|l|r|r|r|}
\hline \multicolumn{4}{|l|}{ HbT p-values for Word Reception paradigm } \\
\hline Channel & \multicolumn{3}{|c|}{ Stimuli Comparison } \\
\hline & ${ }^{*}$ FA v. ${ }^{*}$ RA & *FA v. Rest & ${ }^{* *}$ RA v. Rest \\
\hline L1 & 0.0156 & 0.0043 & 0.0000 \\
\hline L2 & 0.0000 & 0.0000 & 0.0000 \\
\hline L3 & 0.0414 & 0.0000 & 0.0000 \\
\hline L4 & 0.0000 & 0.0000 & 0.0000 \\
\hline L5 & 0.0107 & 0.0185 & 0.0000 \\
\hline L6 & 0.0000 & 0.0000 & 0.0000 \\
\hline L7 & 0.0000 & 0.0000 & 0.0000 \\
\hline L8 & 0.0000 & 0.0000 & 0.0000 \\
\hline R1 & 0.0000 & 0.0024 & 0.0000 \\
\hline R2 & 0.0000 & 0.0000 & 0.0000 \\
\hline R3 & 0.0000 & 0.0008 & 0.0000 \\
\hline R4 & 0.0000 & 0.0000 & 0.0000 \\
\hline R5 & 0.0000 & 0.0000 & 0.0000 \\
\hline R6 & 0.0005 & 0.0000 & 0.0005 \\
\hline R7 & 0.0049 & 0.0000 & 0.0000 \\
\hline R8 & 0.0000 & 0.0000 & 0.0000 \\
\hline \multicolumn{2}{|r|}{${ }^{*}$ FA $=$ Forward Audio, ${ }^{* *}$ RA $=$ Reverse Audio } \\
\hline
\end{tabular}

Table F3: A table of the three paired p-test results for Total hemoglobin (HbT) during the Word Reception Paradigm. 


\begin{tabular}{|c|c|c|c|}
\hline \multicolumn{4}{|c|}{ HbO 3 paired P-values for Word Reception Paradigm } \\
\hline Region & & Stimuli Comparison & \\
\hline & FA v. RA & FA v. Rest & RA v. Rest \\
\hline Left Anterior & 0.000 & 0.002 & 0.000 \\
\hline Left Posterior & 0.000 & 0.000 & 0.000 \\
\hline Right Anterior & 0.000 & 0.000 & 0.000 \\
\hline Right Posterior & 0.000 & 0.000 & 0.000 \\
\hline
\end{tabular}

Table F4: A table of the three paired p-test results for Oxy-hemoglobin ( $\mathrm{HbO})$ during the regional Word Reception Paradigm.

\begin{tabular}{|c|c|c|c|}
\hline \multicolumn{4}{|c|}{ HbR 3 paired $\mathrm{P}$-values for Word Reception Paradigm } \\
\hline Region & & Stimuli Comparison & \\
\hline & FA v. RA & FA v. Rest & RA v. Rest \\
\hline Left Anterior & 0.000 & 0.031 & 0.000 \\
\hline Left Posterior & 0.000 & 0.040 & 0.000 \\
\hline Right Anterior & 0.000 & 0.000 & 0.000 \\
\hline Right Posterior & 0.000 & 0.000 & 0.000 \\
\hline
\end{tabular}

Table F5: A table of the three paired p-test results for Deoxy-hemoglobin (HbR) during the regional Word Reception Paradigm.

\begin{tabular}{|l|r|r|rr|}
\hline \multicolumn{5}{|c|}{ HbT 3 paired P-values for Word Reception Paradigm } \\
\hline Region & \multicolumn{3}{|c|}{ Stimuli Comparison } \\
\hline & FA v. RA & & FA v. Rest & RA v. Rest \\
\hline Left Anterior & & 0.000 & 0.001 & 0.000 \\
\hline Left Posterior & & 0.001 & 0.000 & 0.000 \\
\hline Right Anterior & & 0.001 & 0.000 & 0.000 \\
\hline Right Posterior & & 0.000 & 0.004 & 0.000 \\
\hline
\end{tabular}

Table F6: A table of the three paired p-test results for Total hemoglobin ( $\mathrm{HbT})$ during the regional Word Reception Paradigm. 


\section{REFERENCES}

1. H. Bortfield, E. Wruck, D.A Boas. "' Assessing infants cortical response to speech using near infrared spectroscopy"." Neuroimage, 2007: 407-415.

2. G. Strangman, D. Boas, J.P Sutton, "Non-invasive neuroimaging using near infrared light.” Biol. Psychiatry, 2002:679-693.

3. Huppert, T.J., Diamond, S.G., Franceschini, M.A.,Boas,D. "HomER: a review of timeseries analysis methods for near infrared spectroscopy of the brain." Applied Optics, 2009: 280-298.

4. Selb,J., Scott, J.J, Franceschini, M.A, Sorenson, A.G, Boas, D.A, " Improved Sensitivity to cerebral hemodynamics during brain activation with a time gated optical system: analytical model and experimental validation," J. Biomed Opt. 10, 011013 (2005).

5. Patterson, M.S, Moulton, J.D, Wilson, B.C, "Frequency domain reflectance for the determination of the scattering and absorption properties of tissue," Appl. Opt. 30, 44744476(1991).

6. Tromberg, B.J, Svaasand, L.O, Tsay, T.-T., and Haskell, R.C, " Properties of photon density waves in multiple-scattering media," Appl. Opt. 32, 607-616(1993).

7. Gratton, E., Fantini, S., Franceschini, M.A, Gratton, G. , and Fabiani, M., "Measurements of scattering and absorption changes in muscle and brain," Philos. Trans. R. Soc. London Ser. B352, 727-735 (1997).

8. Y. Fukui, Y. Ajichi, and E. Okada, "Monte Carlo prediction of near-infrared light propagation in realistic adult and neonatal head models," Appl. Opt. 42, 2881-2887 (2003).

9. E. Okada and D. T. Delpy, "Near-infrared light propagation in an adult head model. II. Effect of superficial tissue thicknesson the sensitivity of the near-infrared spectroscopy signal,” Appl. Opt. 42, 2915-2922 (2003).

10. D. Boas, J. Culver, J. Stott, and A. Dunn, "Three dimensional Monte Carlo code for photon migration through complex heterogeneous media including the adult human head," Opt. Express 10, 159-170 (2002).

11. L. Wang, S. L. Jacques, and L. Zheng, "MCML-Monte Carlo modeling of light transport in multi-layered tissues," Comput. Methods Programs Biomed. 47, 131-146 (1995). 
12. M. Hiraoka, M. Firbank, M. Essenpreis, M. Cope, S. R. Arridge, P. van der Zee, and D. T. Delpy, "A Monte Carlo investigation of optical pathlength in inhomogeneous tissue and its application to near-infrared spectroscopy," Phys.Med. Biol. 38, 1859-1876 (1993).

13. S. T. Flock, M. S. Patterson, B. C. Wilson, and D. R. Wyman, "Monte Carlo modeling of light propagation in highly scattering tissue-I: Model predictions and comparison with diffusion theory," IEEE Trans. Biomed. Eng. 36, 1162-1168 (1989).

14. S. T. Flock, B. C. Wilson, and M. S. Patterson, "Monte Carlo modeling of light propagation in highly scattering tissues - II: Comparison with measurements in phantoms," IEEE Trans. Biomed. Eng. 36, 1169-1173 (1989).

15. A. Custo, W. M. Wells III, A. H. Barnett, E. M. Hillman, and D. A. Boas, "Effective scattering coefficient of the cerebral spinal fluid in adult head models for diffuse optical imaging,"Appl. Opt. 45, 4747-4755 (2006).

16. M. Cope, D. T. Delpy, E. O. Reynolds, S. Wray, J. Wyatt, and P.van der Zee, "Methods of quantitating cerebral near infrared spectroscopy data," Adv. Exp. Med. Biol. 222, 183189 (1988).

17. D. T. Delpy, M. Cope, P. van der Zee, S. Arridge, S. Wray, and J. Wyatt, "Estimation of optical pathlength through tissue from direct time of flight measurement," Phys. Med. Biol. 33, 1433-1442 (1988).

18. Jöbsis, F.F., "Noninvasive, infrared monitoring of cerebral and myocardial oxygen sufficiency and circulatory parameters," Science 198, 1264-1267 (1977).

19. H. R. Heekeren, H. Obrig, R. Wenzel, K. Eberle, J. Ruben, K. Villringer, R. Kurth, and A. Villringer,"Cerebral haemoglobin oxygenation during sustained visual stimulation-a near infrared spectroscopy study,” Philos. Trans. R. Soc. London Ser. B 352, 743-750 (1997).

20. J. H. Meek, C. E. Elwell, M. J. Khan, J. Romaya, J. S. Wyatt, D. T. Delpy, and S. Zeki, "Regional changes in cerebral haemodynamics as a result of a visual stimulus measured by near infrared spectroscopy," Proc. R. Soc. London Ser. B 261, 351-356 (1995).

21. J. Ruben, R. Wenzel, H. Obrig, K. Villringer, J. Bernarding, C. Hirth, H. Heekeren, U. Dirnagl, and A. Villringer, "Haemoglobin oxygenation changes during visual stimulation in the occipital cortex," Adv. Exp. Med. Biol. 428, 181-187 (1997).

22. K. Sakatani, S. Chen, W. Lichty, H. Zuo, and Y. P. Wang, "Cerebral blood oxygenation changes induced by auditory stimulation in newborn infants measured by near infrared spectroscopy," Early Hum. Dev. 55, 229-236 (1999). 
23. S. Chen, K. Sakatani, W. Lichty, P. Ning, S. Zhao, and H. Zuo, “Auditory-evoked cerebral oxygenation changes in hypoxicischemic encephalopathy of newborn infants monitored by near infrared spectroscopy," Early Hum. Dev. 67, 113-121 (2002).

24. P. Zaramella, F. Freato, A. Amigoni, S. Salvadori, P. Marangoni, A. Suppjei, B. Schiavo, and L. Chiandetti, "Brain auditory activation measured by near-infrared spectroscopy (NIRS) in neonates," Pediatr. Res. 49, 213219 (2001).

25. M. A. Franceschini, S. Fantini, J. H. Thompson, J. P. Culver, and D. A. Boas, "Hemodynamic evoked response of the sensorimotor cortex measured noninvasively with near-infrared optical imaging," Psychophysiology 40, 548-560 (2003).

26. H. Obrig, T. Wolf, C. Doge, J. J. Hulsing, U. Dirnagl, and A. Villringer, "Cerebral oxygenation changes during motor and somatosensory stimulation in humans, as measured by near-infrared spectroscopy,” Adv. Exp. Med. Biol. 388, 219- 224 (1996).

27. H. Obrig, C. Hirth, J. G. Junge-Hulsing, C. Doge, T. Wolf, U. Dirnagl, and A. Villringer, "Cerebral oxygenation changes in response to motor stimulation," J. Appl. Physiol. 81, 1174-1183 (1996).

28. W. N. Colier, V. Quaresima, B. Oeseburg, and M. Ferrari, "Human motor-cortex oxygenation changes induced by cyclic coupled movements of hand and foot," Exp. Brain. Res. 129, 457-461 (1999).

29. C. Hirth, H. Obrig, K. Villringer, A. Thiel, J. Bernarding, W. Muhlnickel, H. Flor, U. Dirnagl, and A. Villringer, "Noninvasive functional mapping of the human motor cortex using near-infrared spectroscopy," NeuroReport 7, 1977-1981 (1996).

30. A.Kleinschmidt, H. Obrig, M. Requardt, K. D. Merboldt, U. Dirnagl, A. Villringer, and J. Frahm, "Simultaneous recording of cerebral blood oxygenation changes during human brain activation by magnetic resonance imaging and near-infrared spectroscopy," J. Cereb. Blood Flow Metab. 16, 817-826 (1996).

31. M. Kameyama, M. Fukuda, Y. Yamagishi, T. Sato, T. Uehara, M. Ito, T. Suto, and M. Mikuni, "Frontal lobe function in bipolar disorder: a multichannel near-infrared spectroscopy study," NeuroImage 29, 172-184 (2006).

32. S. Nagamitsu, M. Nagano, Y. Yamashita, S. Takashima, and T. Matsuishi, "Prefrontal cerebral blood volume patterns while playing video games-a near-infrared spectroscopy study,” Brain Dev. 28, 315-321 (2006).

33. M. Okamoto, M. Matsunami, H. Dan, T. Kohata, K. Kohyama, and I. Dan, "Prefrontal activity during taste encoding: an fNIRS study," NeuroImage 31, 796-806 (2006). 
34. S. Sumitani, T. Tanaka, S. Tayoshi, K. Ota, N. Kameoka, M. Morimune, S. ShibuyaTayoshi, S. Kinouchi, S. Ueno, and T. Ohmori, "Hemodynamic changes in the prefrontal cortex during mental works as measured by multi channel near infrared spectroscopy (NIRS),” J. Med. Invest. 52(Suppl.), 302-303 (2005).

35. Zhang,Y.J, Lu, C.M., Biswal,B.B., Zhang, Y.F., Peng,D.F, Zhu, C.Z., "Detecting resting state functional connectivity in the language system using functional near infrared spectroscopy" Journ. Of Biomed. Optics, 15(4), 1-8(2010).

36. H. Sato, T. Takeuchi, and K. L. Sakai, "Temporal cortex activation during speech recognition: an optical topography study," Cognition 73, B55-66 (1999).

37. Cannestra, A. F, Wartenburger, I., Obrig, H.M, Villringer, A., Toga, A.W, "Functional Assessment of Broca's Area Using Near Infrared Spectroscopy in Humans." Neuro Rep. , 14(15), 1961-1965(2003).

38. Ehlis, A. H., Herrmann, M.J, Plichta, M.M, Fallgatter, A.J "Cortical Activation during two verbal fluency tasks in schizophrenic patients and healthy controls as assessed by multichannel NIRS.” Psy. Re.: Neuroim., 156(1), 1-13(2007).

39. Quaresima, V. D., Ferrari, M., Van Der Sluiis, C.P., Menssen, J., Colier, W.N., "Lateral Frontal Cortex Oxygenation Changes During Translation and Language Switching Revealed by Non Invasive Near Infrared Multipoint Measurements." Brain Res. Bull., 59(3), 235-243(2002).

40. Brauer, J, Neumann,J., Friederici.,A.D, “Temporal dynamics of perisylvian activation during language processing in children and adults." Neuroim. , 41(4), 1484-1492(2008).

41. Schecklmann, M., Ehlis, A.C, Plichta, M.M., Fallgatter, A.J., "Functional Near Infrared Spectroscopy: A long term reliable tool for measuring brain activity during verbal fluency." NeuroIm. , 43(1), 147-155(2008).

42. Watanabe, F. M., Maki, A., Kawaguchi, F., Takashiro, K., Yamashita, Y., Koizumi, H., Mayanagi, Y., "Non-Invasive Assessment of Language Dominance with Near Infrared Spectroscopy Mapping." Nuerosci. Lett. ,256(1) ,49-52(1998).

43. J.F Demonet, F. Chollet, S. Ramsay, D. Cardebat, J.L Nespoulous, R. Wise, A. Rascol, and R. Frackowiak, " The anatomy of phonological and semantic processing in normal subjects.” Brain, 1753-1768(1992).

44. C. Bloch, A. Kaiser, E. Keunzli, D. Zappatore, S. Haller, R. Franceschini, G. Luedi, E. Wilhelm-Radue, C. Nitsch. "The age of second language acquisition determines the variability in activation elicited by narration in three languages in Broca's and Wernicke's area." Neuropsychologia, 2009. 
45. K. Wagner, L. Frings, J. Spreer, A. Buller, R. Everts, U. Halsband, A.S Bonhange. "Differential effect of side of temporal lobe epilepsy on lateralization of hippocampal, tempolateral, and inferior frontal activation patterns during a verbal episodic memory task." Epilepsy \& Behavior, 2008: 382-387.

46. S.M Wilson, I. Molnar-Szackacs, M. Iacoboni. "Beyond Superior Temporal Cortex: Inter- subject Correlations in Narrative Speech Comprehension ." Cerebral Cortex, 2008: 230-242.

47. Hampson, M., Peterson, B.S., Skularski, P., Gatenby, J.C., Gore, J.C., "Detection of functional connectivity using temporal correlations in MR images". Hum Brain Mapp. 15(4), 247-262(2002).

48. Bartels, A., Semir, Z., “ Brain dynamics during natural viewing conditions- A new guide for mapping connectivity in vivo". Neuroimage (24),339-349(2005).

49. Hoshida, T., Hirabayashi, H., Kaido, T., Nakase, H., Kameguchi, S., Sakaki, T., “ Functional brain mapping detected by cortical stimulation using chronically implanted electrodes". International Congress Series (1232), 877-881(2002).

50. Suto T., Fukuda M., Ito M., Uehara T., Masahiko M., “ Multichannel near-infrared Spectroscopy in depression and Schizophrenia: Cognitive Brain Activation Study." Biol. Psych. 2004:55:501-511.

51. A.C Ehlis, M.J Herrmann, A.Wagener, A.J. Fallgatter. "" Multi-channel near-infrared spectroscopy detects specific interior-frontal activation during incongruent Stroop trials"." Biol. Psychology, 2005: 315-331.

52. Lurito, J.T., Dzemidzic, M., Mathews, V.P., Lowe, M.J., Kareken, D.A., Phillips, M.D, Wang, Y., "Comparison of hemispheric lateralization using four language tasks." Neuroimage 11:S358(2000).

53. N.F. Watson, C. Dodrill, D. Farrell, M.D. Holmes, J.W. Miller, Determination of language dominance with near-infrared spectroscopy: comparison with the intracarotid amobarbital procedure, Seizure. 13 (2004) 399-402.

54. Gevins, A.S, Shaffer, R.E, Doyle, J.C, Cutillo, B.A, Tannehill, R.S, Bressler, S.L, "Shades of thought: Shifting lateralization of human brain electrical patterns during brief visuomotor tasks." Science 220(1), 97-99(1983).

55. Cohen, J. (1977) Statistical Power Analysis for the Behaviorial Sciences. Rev Edition. New York: Academic Press (Book). 
56. Cannestra AF, Bookheimer SY, Pouratian N, et al. "Temporal and Topographical characterization of Language cortices using Intraoperative Optical Intrinsic Signals." Neuroimage 2000:12:41-54.

57. K.M Petersson, C. Forkstam, M.Ingvar, “Artificial syntactic violations activate Broca's Region.” Cognitive Science,383-407(2004).

58. C.D Frith, K.J Friston, P.F Liddle, R.S.J Frackowiak. "A PET study of word Findings." Neurophysiologica, 1991.

59. Y.Noguchi, T. Takeuchi, K.L Sakai, "Lateralized activation in the inferior frontal cortex during syntactic processing: event related optical topography study." Human Brain Mapp., 2002:89-99.

60. T. Nakai, K. Matsuo, C. Kato. "A functional magnetic resonance imaging study of listening comprehension of languages in human at 3 tesla-comprehension level and activation of the language areas." Neuroscience Letters, 1999: 33-36.

61. Caplan, R., Dupretto, M., Mazziotta, J.C., "An fMRI study of discourse coherence”. Neuroimage 11:S96(2000).

62. Paquette, N., Gonzalez-Frankenberger, B., Vannasing, P., Tremblay, J., Florea, O., Beland, R., Lepore, F. , Lassonde, M., "Lateralization of Receptive Language Function Using near Infrared Spectroscopy” Nuerosci \& Med., 1, 64-70, (2010).

63. L. Fadiga, L. Craighero. "Hand Actions and Speech Representation in Broca's Area." Cortex, 2006: 486-490.

64. S.Y Bookheimer, T.A Zeffiro, T. Blaxton, W. Gaillard, W. Theodore. "Regional Cerebral blood flow during object naming and word reading." Hum. Brain Mapping, 1995: 93106.

65. Noonan, S.K, Haist, F., Muller, R.A, "Aberrant functional connectivity in Autism: Evidence from low frequency BOLD signal fluctuations." J. Brainres.12(2008)48-63.

66. Petrides, M., Pandya, D.N., “ Comparative cytoarchitectonic analysis of the human and the macque ventrolateral prefrontal cortex and corticocortical connection patterns in the monkey". Eur. J. Neurosci. 16(2), 291-310.

67. Scott, S.K., Johnsrude, I.S., "The neuroanatomical and functional organization of speech perception”. Trends Neurosci. 26(2), 100-107.

68. Corbetta, M., "Functional connectivity and neurological recovery" Dev.Psychobiol. (2010). 
69. Meyer, M. A., , Alter, K., Frederici, A. "Functional MR Imaging exposes differential brain responses to syntax and prosody during auditory sentence comprehension.", $J$. NeuroLing. ,16(4-5), 277-300(2003). 